\title{
Algebras of invariant differential operators on unit sphere bundles over two-point homogeneous Riemannian spaces
}

\author{
A.V. Shchepetilov \\ Department of Physics, Moscow State University, \\ 119992 Moscow, Russia*
}

\begin{abstract}
Let $G$ be the identity component of the isometry group for an arbitrary curved two-point homogeneous space $M$. We consider algebras of $G$-invariant differential operators on bundles of unit spheres over $M$. The generators of this algebra and the corresponding relations for them are found. The connection of these generators with two-body problem on two-point homogeneous spaces is discussed.
\end{abstract}

Keywords: two point-homogeneous spaces, invariant differential operators, two-body problem

PACS number: 02.40.Vh, 02.40.Ky, 02.20.Qs, 03.65.Fd

Mathematical Subject Classification: 16S32, 43A85, 22F30, 70F05.

*email: alexey@quant.phys.msu.su 


\section{Introduction}

The property of a differential operator on a smooth manifold $M$ to be invariant with respect to an action of some group $G$ (especially a Lie group) on $M$ plays a great role in mathematical physics since it helps select physically significant operators. The algebra $\operatorname{Diff}(M)$ of all $G$-invariant differential operators with complex or real coefficients on $M$ gives the material for constructing $G$-invariant physical theories on $M$. Properties of such theory are in close connection with properties of the algebra $\operatorname{Diff}(M)$.

A homogeneous smooth manifold $M$ of the Lie group $G$ is called commutative space, if the algebra $\operatorname{Diff}(M)$ is commutative. The well known example of a commutative space is the symmetric space of the rank $l$. Recall that the rank of a symmetric space is the dimension of its maximal flat completely geodesic submanifold. The commutative algebra $\operatorname{Diff}(M)$ for this space is generated by $l$ independent commutative generators [1. Particularly, for symmetric spaces of the rank one (which are the same as two-point homogeneous spaces) the algebra $\operatorname{Diff}(M)$ is generated by the Laplace-Beltrami operator. Also, the class of commutative spaces contains weakly symmetric spaces [2].

There are known only some sporadic examples of noncommutative algebras $\operatorname{Diff}(M)$ (see, for example Ch.2, 3] $)$. One of these example is the noncommutative algebra $\operatorname{Diff}\left(M_{1}\right)$ for $M_{1}=\mathbf{O}_{0}(1, n) / \mathbf{S O}(n-1)$ studied in 4], where $\mathbf{O}_{0}(1, n)$ is the identity component for the group $\mathbf{O}(1, n)$. In that paper the space $M_{1}$ was interpreted as the total space for the bundles of unit spheres over the hyperbolic space $\mathbf{H}^{n}(\mathbb{R})$. Denote the total space of the bundle of unit spheres over a Riemannian space $M$ by $M_{\mathbb{S}}$.

The space $\mathbf{H}^{n}(\mathbb{R})$ is a representative of the class of two-point homogeneous Riemannian spaces (TPHRS) for which any pair of points can be transformed by means of appropriate isometry to any other pair of points with the same distance between them. Equivalently, these spaces are characterized by the property that the natural action of the isometry group on the bundle of unit spheres over them are transitive. Thus the natural problem arises: "describe the algebras $\operatorname{Diff}\left(M_{\mathbb{S}}\right)$ for the bundle of unit spheres over all TPHRS M".

From the point of view of the two-body problem in classical and quantum mechanics, TPHRS are characterizes by the property that the distance between particles is the only invariant of the isometry group $G$ in the configuration space. The space $M_{\mathbb{S}}$ is isomorphic to an orbit in general position for the symmetry group $G$ of the two-body problem on the TPHRS $M$ acting in the configuration space of this problem. Due to the two-point homogeneity of $M$ the codimension of these orbits is one. Thus, for the two-body problem in TPHRS there is the degree of freedom corresponding to the distance between particles; other degrees of freedom correspond to the homogeneous manifold and can be described in terms of the symmetry group $G$.

In the paper [5] the polynomial expression for the Hamiltonian $\hat{H}$ of the quantum mechanical two-body problem on an arbitrary TPHRS $M$ was found through the radial differential operator and elements of $\operatorname{Diff}\left(M_{\mathbb{S}}\right)$. In the present paper the generators of algebras $\operatorname{Diff}\left(M_{\mathbb{S}}\right)$ and the corresponding relations for them are found for all curved twopoint homogeneous spaces $M$. Some properties of these generators are discussed.

This paper is organized as follows. In section 2 we perform the necessary information on invariant differential operators on homogeneous spaces. We recall the classification of TPHRS in section 3 In section 4 we specify the construction of invariant differential operators for the space $M_{\mathbb{S}}$, where $M$ is a two-point homogeneous Riemannian space. There are found some generators common for all Diff $\left(M_{\mathbb{S}}\right)$. In section 5 the model of the quaternion projective space $\mathbf{P}^{n}(\mathbb{H})$ is described. In section 6 the generators for the algebra $\operatorname{Diff}\left(\mathbf{P}^{n}(\mathbb{H})_{\mathbb{S}}\right)$ are calculated and by the formal correspondence the analogous generators of the algebra Diff $\left(\mathbf{H}^{n}(\mathbb{H})_{\mathbb{S}}\right)$ is obtained. The corresponding relations for these algebras are found in section 7 In section 8 we consider from the same point of view algebras $\operatorname{Diff}\left(\mathbf{P}^{n}(\mathbb{C})_{\mathbb{S}}\right), \operatorname{Diff}\left(\mathbf{H}^{n}(\mathbb{C})_{\mathbb{S}}\right)$ and in section 9 the algebras $\operatorname{Diff}\left(\mathbf{P}^{n}(\mathbb{R})_{\mathbb{S}}\right)$, Diff $\left(\mathbf{S}_{\mathbb{S}}^{n}\right)$. In the 
section[10 there is a description of the Cayley plane $\mathbf{P}^{2}(\mathbb{C} a)$ through the exceptional Jordan algebra $\mathfrak{h}_{3}(\mathbb{C} a)$. In section 11 the generators for the algebra $\operatorname{Diff}\left(\mathbf{P}^{2}(\mathbb{C} a)_{\mathbb{S}}\right)$ are calculated and by the formal correspondence the analogous generators of the algebra $\operatorname{Diff}\left(\mathbf{H}^{2}(\mathbb{C} a)_{\mathbb{S}}\right)$ is obtained. The corresponding relations for these algebras are found in section 12

The connection of constructed generators with two-body problem on two-point homogeneous spaces is discussed in section 13

In appendix $\mathrm{A}$ we describe the technique for calculating the commutative relations for algebras of differential operators under consideration and in appendix B one interesting fact for an arbitrary TPHRS is proved.

\section{Invariant differential operators on homogeneous spaces}

Let $G$ be a Lie group, $M$ be a Riemannian $G$-homogeneous left space, $x_{0} \in M, K \subset G$ be the stationary subgroup of the point $x_{0} \in M$, and $\mathfrak{k} \subset \mathfrak{g} \equiv T_{e} G$ be the corresponding Lie algebras. Choose a subspace $\mathfrak{p} \subset \mathfrak{g}$ such that $\mathfrak{g}=\mathfrak{p} \oplus \mathfrak{k}$ (a direct sum of linear spaces). The space $\mathfrak{p}$ can be identified with the tangent space $T_{x_{0}} M$.

The stationary subgroup $K$ is compact, since it is also the subgroup of the group $\mathbf{S O}(n)$. By the averaging on the group $K$ we can define a $\operatorname{Ad}_{K}$-invariant scalar product $\langle\cdot, \cdot\rangle$ on $\mathfrak{g}$ and choose the subspace $\mathfrak{p}$ orthogonal to $\mathfrak{k}$ with respect to this product 3 , 6]. In this case we have the inclusion $\operatorname{Ad}_{K}(\mathfrak{p}) \subset \mathfrak{p}$, i.e. the space $M$ is reductive.

Identify the space $M$ with the factor space of left conjugate classes of the group $G$ with respect to the subgroup $K$. Let $\pi: G \rightarrow G / K$ be the natural projection.

Let $S(V)$ be a graded symmetric algebra over a finite dimensional complex space $V$, i.e. a free commutative algebra over the field $\mathbb{C}$, generated by elements of any basis of $V$. The adjoint action of the group $G$ on $\mathfrak{g}$ can be naturally extended to the action of $G$ on the algebra $S(\mathfrak{g})$ according to the formula:

$$
\operatorname{Ad}_{q}: Y_{1} \cdot \ldots \cdot Y_{i} \rightarrow \operatorname{Ad}_{q}\left(Y_{1}\right) \cdot \ldots \cdot \operatorname{Ad}_{q}\left(Y_{i}\right), Y_{1}, \ldots, Y_{i} \in \mathfrak{g} .
$$

Denote by $\mathfrak{g}^{K}$ the set of all Ad-invariants in $S(\mathfrak{g})$.

The main result of the invariant differential operators theory is the existence of the one to one correspondence between the algebra $\operatorname{Diff}(M)$ and the set $\mathfrak{p}^{K}$ of all $\mathrm{Ad}_{K}$-invariants in $S(\mathfrak{p})$ [3. For our purpose the next version $[2$ of this result is more convenient. Let $U(\mathfrak{g})$ be the universal enveloping algebra with the standard filtration for the Lie algebra $\mathfrak{g}$ and $U(\mathfrak{g})^{K}$ be it's subalgebra, consisting of all $\operatorname{Ad}_{K}$-invariant elements in $U(\mathfrak{g})$. Let $\mu$ be the linear mapping of $S(\mathfrak{p})$ into $U(\mathfrak{g})$, according to the formula

$$
\mu\left(Y_{1} \cdot \ldots \cdot Y_{p}\right)=\frac{1}{p !} \sum_{\sigma \in \mathfrak{S}_{p}} Y_{\sigma(1)} \cdot \ldots \cdot Y_{\sigma(p)}
$$

where on the left side the element $Y_{1} \cdot \ldots \cdot Y_{p}$ is supposed to be in $S(\mathfrak{p})$ and on the right side it is supposed to be in $U(\mathfrak{g})$. Here $\mathfrak{S}_{p}$ is the permutation group of $p$ elements. Obviously, $\mu$ is injective.

Let $U(\mathfrak{g}) \mathfrak{k}$ be the left ideal in $U(\mathfrak{g})$ generated by $\mathfrak{k}$ and $(U(\mathfrak{g}) \mathfrak{k})^{K}$ be the set of all $\operatorname{Ad}_{K^{-}}$ invariant elements in $U(\mathfrak{g})^{\mathfrak{k}}$. The set $\left(U(\mathfrak{g})_{\mathfrak{k}}\right)^{K}$ is a two-sided ideal in $U(\mathfrak{g})^{K}$ since for elements $f \in \mathfrak{k}$ and $g \in U(\mathfrak{g})^{K}$ we have $f g=\operatorname{ad}_{f} g+g f=g f$. Also $\mu\left(\mathfrak{p}^{K}\right) \subset U(\mathfrak{g})^{K}$, because $M$ is reductive. Hence we can define the factor algebra $U(\mathfrak{g})^{K} /(U(\mathfrak{g}) \mathfrak{k})^{K}$. Let $\eta: U(\mathfrak{g})^{K} \rightarrow U(\mathfrak{g})^{K} /(U(\mathfrak{g}) \mathfrak{k})^{K}$ be the canonical projection.

Theorem 1 ([2]). The algebras $\operatorname{Diff}(M)$ and $U(\mathfrak{g})^{K} /(U(\mathfrak{g}) \mathfrak{k})^{K}$ are isomorphic.

Every element of $U(\mathfrak{g})^{K} /(U(\mathfrak{g}) \mathfrak{k})^{K}$ has the unique representative from $\mathfrak{p}^{K}$ or equivalently from $\mu\left(\mathfrak{p}^{K}\right)$. We can get the relations in $\operatorname{Diff}(M)$ operating in $U(\mathfrak{g})^{K}$ modulo 
$(U(\mathfrak{g}) \mathfrak{k})^{K}$. This approach leads to simpler calculations than the operations through local coordinates on $M$ (like in 4) that gives quite cumbersome calculations even in the relatively simple case of $M=\mathbf{H}^{n}(\mathbb{R})_{\mathbb{S}}$.

Below we are interested in the representation of the associative algebra $\operatorname{Diff}(M)$ by it's generators and corresponding relations. Let $\left\{g_{i}\right\}$ be a set of generators of the commutative subalgebra $\mathfrak{p}^{K} \subset S(\mathfrak{p})$. Not loosing generality we can suppose that all $g_{i}$ are homogeneous elements w.r.t. the grading of $S(\mathfrak{p})$. Then the elements $\eta \circ \mu\left(g_{i}\right)$ generate the algebra $\operatorname{Diff}(M)$.

Relations for the elements $\eta \circ \mu\left(g_{i}\right)$ are of two types. First type consists of relations induced by relations in $U(\mathfrak{g})$. Due to the universality of $U(\mathfrak{g})$ all these relation are commutative ones, induced by the Lie operation in $\mathfrak{g}$. They are reduced to commutative relations of the simplest form: $\left[D_{1}, D_{2}\right]=\tilde{D}$, where the operators $D_{1}, D_{2} \in \operatorname{Diff}(M)$ have degrees $m_{1}$ and $m_{2}$ respectively and the degree of $\tilde{D} \in \operatorname{Diff}(M)$ is less or equal $m_{1}+m_{2}-1$.

Suppose now that there is a relation in $U(\mathfrak{g})$ of the form:

$$
P\left(\eta \circ \mu\left(g_{1}\right), \ldots, \eta \circ \mu\left(g_{k}\right)\right)=0
$$

or equivalently

$$
P\left(\mu\left(g_{1}\right), \ldots, \mu\left(g_{k}\right)\right)=\tilde{D},
$$

where $P$ is a polynomial and $\tilde{D} \in\left(U(\mathfrak{g})_{\mathfrak{k}}\right)^{K}$. Using the commutative relations for $\mu\left(g_{i}\right), i=$ $1, \ldots, k$ we can reduce the polynomial $P$ to the polynomial $P_{1}$, symmetric w.r.t. all permutations of it's arguments, and equation (1) becomes:

$$
P_{1}\left(\mu\left(g_{1}\right), \ldots, \mu\left(g_{k}\right)\right)=D^{*}
$$

$D^{*} \in(U(\mathfrak{g}) \mathfrak{k})^{K}$. After this reduction the relation (2) may be trivial: $P_{1}=0, D^{*}=0$. It means that (11) is the commutative relation. Suppose that relation (2) is nontrivial. Consider the sum $P_{2}\left(t_{1}, \ldots, t_{k}\right)$ of monomials with the highest total degree from polynomial $P_{1}\left(t_{1}, \ldots, t_{k}\right)$ with commutative variables $t_{1}, \ldots, t_{k}$. Due to the symmetry of $P_{1}$ the polynomial $P_{2}\left(t_{1}, \ldots, t_{k}\right)$ is nontrivial. On the other hand from (2) we obtain that $P_{2}\left(g_{1}, \ldots, g_{k}\right)=0$ due to the expansion $\mathfrak{g}=\mathfrak{p} \oplus \mathfrak{k}$. Thus every relation in the algebra $U(\mathfrak{g})^{K} /(U(\mathfrak{g}) \mathfrak{k})^{K}$ w.r.t. it's generators $\eta \circ \mu\left(g_{1}\right), \ldots, \eta \circ \mu\left(g_{k}\right)$ modulo commutative relations is in one to one correspondence with the relations for homogeneous generators $g_{1}, \ldots, g_{k}$ of commutative algebra $\mathfrak{p}^{K}$. We call such relations the relations of the second type.

The filtration of the algebra $U(\mathfrak{g})$ induces the filtration of the algebra $\operatorname{Diff}(M)$ which coincides with the natural filtration of $\operatorname{Diff}(M)$ as the algebra of differential operators.

For simplicity throughout the whole paper we consider invariance of differential operators only w.r.t. the identity component of a whole isometry group.

\section{Two-point homogeneous Riemannian spaces}

The classification of two-point homogeneous Riemannian spaces can been found in [7, 8], (see also [9], 10], 11]), and is as follows:

1. the Euclidean space $\mathbf{E}^{n}$;

2. the sphere $\mathbf{S}^{n}$;

3. the real projective space $\mathbf{P}^{n}(\mathbb{R})$;

4. the complex projective space $\mathbf{P}^{n}(\mathbb{C})$;

5. the quaternion projective space $\mathbf{P}^{n}(\mathbb{H})$; 
6. the Cayley projective plane $\mathbf{P}^{2}(\mathbb{C} a)$;

7. the real hyperbolic space (Lobachevski space) $\mathbf{H}^{n}(\mathbb{R})$;

8. the complex hyperbolic space $\mathbf{H}^{n}(\mathbb{C})$;

9. the quaternion hyperbolic space $\mathbf{H}^{n}(\mathbb{H})$;

10. the Cayley hyperbolic plane $\mathbf{H}^{2}(\mathbb{C} a)$.

The isometry groups for all these spaces except Cayley planes are classical and for the Cayley planes they are two real forms of the complex special simple group $\mathbf{F}_{4}$.

For the Lie algebra $\mathfrak{g}$ of the isometry group of the compact two-point homogeneous space $M$ there is the following general expansion [5, 12, [13, which is the specification of the expansion $\mathfrak{g}=\mathfrak{p} \oplus \mathfrak{k}$ from section 2

Proposition 1. The algebra $\mathfrak{g}$ admits the following expansion into the direct sum of subspaces:

$$
\mathfrak{g}=\mathfrak{a} \oplus \mathfrak{k}_{0} \oplus \mathfrak{k}_{\lambda} \oplus \mathfrak{k}_{2 \lambda} \oplus \mathfrak{p}_{\lambda} \oplus \mathfrak{p}_{2 \lambda}
$$

where $\operatorname{dim} \mathfrak{a}=1, \lambda$ is a nontrivial linear form on the space $\mathfrak{a}$, $\operatorname{dim} \mathfrak{k}_{\lambda}=\operatorname{dim} \mathfrak{p}_{\lambda}=q_{1}$, $\operatorname{dim} \mathfrak{k}_{2 \lambda}=\operatorname{dim} \mathfrak{p}_{2 \lambda}=q_{2}, \mathfrak{p}=\mathfrak{a} \oplus \mathfrak{p}_{\lambda} \oplus \mathfrak{p}_{2 \lambda}, \mathfrak{k}=\mathfrak{k}_{0} \oplus \mathfrak{k}_{\lambda} \oplus \mathfrak{k}_{2 \lambda} ; q_{1}, q_{2} \in\{0\} \cup \mathbb{N}$, the subalgebra $\mathfrak{a}$ is the maximal commutative subalgebra in the subspace $\mathfrak{p}$. Here $\mathfrak{k}$ is a stationary subalgebra, corresponding to some point $x_{0} \in M$. All summands in (3) are $\operatorname{ad}_{\mathfrak{k}_{0}}$-invariant; in particular $\mathfrak{k}_{0}$ is a subalgebra of $\mathfrak{k}$.

Identified the space $\mathfrak{p}$ with the space $T_{x_{0}} M$. Under this identification the restriction of the Killing form for the algebra $\mathfrak{g}$ onto the space $\mathfrak{p}$ and the scalar product on $T_{x_{0}} M$ are proportional. In particular, the decomposition $\mathfrak{g}=\mathfrak{p} \oplus \mathfrak{k}$ is uniquely determined by the point $x_{0}$. Let $\langle\cdot, \cdot\rangle$ be a scalar product on the algebra $\mathfrak{g}$ such that it is proportional to the Killing form and its restriction onto the subspace $\mathfrak{p} \cong T_{x_{0}} M$ coincides with the Riemannian metric $g$ on $T_{x_{0}} M$. The spaces $\mathfrak{a}, \mathfrak{k}_{0}, \mathfrak{k}_{\lambda}, \mathfrak{p}_{\lambda}, \mathfrak{k}_{2 \lambda}, \mathfrak{p}_{2 \lambda}$ are pairwise orthogonal.

Besides, the following inclusions are valid:

$$
\begin{array}{r}
{\left[\mathfrak{a}, \mathfrak{p}_{\lambda}\right] \subset \mathfrak{k}_{\lambda},\left[\mathfrak{a}, \mathfrak{k}_{\lambda}\right] \subset \mathfrak{p}_{\lambda},\left[\mathfrak{a}, \mathfrak{p}_{2 \lambda}\right] \subset \mathfrak{k}_{2 \lambda},\left[\mathfrak{a}, \mathfrak{k}_{2 \lambda}\right] \subset \mathfrak{p}_{2 \lambda},\left[\mathfrak{a}, \mathfrak{k}_{0}\right]=0,\left[\mathfrak{k}_{\lambda}, \mathfrak{p}_{\lambda}\right] \subset \mathfrak{p}_{2 \lambda} \oplus \mathfrak{a},} \\
{\left[\mathfrak{k}_{\lambda}, \mathfrak{k}_{\lambda}\right] \subset \mathfrak{k}_{2 \lambda} \oplus \mathfrak{k}_{0},\left[\mathfrak{p}_{\lambda}, \mathfrak{p}_{\lambda}\right] \subset \mathfrak{k}_{2 \lambda} \oplus \mathfrak{k}_{0},\left[\mathfrak{k}_{2 \lambda}, \mathfrak{k}_{2 \lambda}\right] \subset \mathfrak{k}_{0},\left[\mathfrak{p}_{2 \lambda}, \mathfrak{p}_{2 \lambda}\right] \subset \mathfrak{k}_{0},\left[\mathfrak{k}_{2 \lambda}, \mathfrak{p}_{2 \lambda}\right] \subset \mathfrak{a},} \\
{\left[\mathfrak{k}_{\lambda}, \mathfrak{k}_{2 \lambda}\right] \subset \mathfrak{k}_{\lambda},\left[\mathfrak{k}_{\lambda}, \mathfrak{p}_{2 \lambda}\right] \subset \mathfrak{p}_{\lambda},\left[\mathfrak{p}_{\lambda}, \mathfrak{k}_{2 \lambda}\right] \subset \mathfrak{p}_{\lambda},\left[\mathfrak{p}_{\lambda}, \mathfrak{p}_{2 \lambda}\right] \subset \mathfrak{k}_{\lambda} .}
\end{array}
$$

Moreover, for any basis $e_{\lambda, i}, i=1, \ldots, q_{1}$ in the space $\mathfrak{p}_{\lambda}$ and any basis $e_{2 \lambda, i}, i=1, \ldots, q_{2}$ in the space $\mathfrak{p}_{2 \lambda}$ there are the basis $f_{\lambda, i}, i=1, \ldots, q_{1}$ in the space $\mathfrak{k}_{\lambda}$ and the basis $f_{2 \lambda, j}, j=$ $1, \ldots, q_{2}$ in the space $\mathfrak{k}_{2 \lambda}$ such that:

$$
\begin{aligned}
{\left[\Lambda, e_{\lambda, i}\right] } & =-\frac{1}{2} f_{\lambda, i},\left[\Lambda, f_{\lambda, i}\right]=\frac{1}{2} e_{\lambda, i},\left[e_{\lambda, i}, f_{\lambda, i}\right]=-\frac{1}{2} \Lambda, \\
\left\langle e_{\lambda, i}, e_{\lambda, j}\right\rangle & =\left\langle f_{\lambda, i}, f_{\lambda, j}\right\rangle=\delta_{i j} R^{2}, i, j=1, \ldots, q_{1}, \\
{\left[\Lambda, e_{2 \lambda, i}\right] } & =-f_{2 \lambda, i},\left[\Lambda, f_{2 \lambda, i}\right]=e_{2 \lambda, i},\left[e_{2 \lambda, i}, f_{2 \lambda, i}\right]=-\Lambda, \\
\left\langle e_{2 \lambda, i}, e_{2 \lambda, j}\right\rangle & =\left\langle f_{2 \lambda, i}, f_{2 \lambda, j}\right\rangle=\delta_{i j} R^{2}, i, j=1, \ldots, q_{2},\langle\Lambda, \Lambda\rangle=R^{2},
\end{aligned}
$$

where the vector $\Lambda \in \mathfrak{a}$ satisfies the conditions $\langle\Lambda, \Lambda\rangle=R^{2},|\lambda(\Lambda)|=\frac{1}{2}$. Here the positive constant $R$ is connected with the maximal sectional curvature $\varkappa_{m}$ of the space $M$ by the formula $\varkappa_{m}=R^{-2}$.

Nonnegative integers $q_{1}$ and $q_{2}$ are said to be multiplicities of the space $M$. They characterize $M$ uniquely up to the exchange $\mathbf{S}^{n} \leftrightarrow \mathbf{P}^{n}(\mathbb{R})$. For the spaces $\mathbf{S}^{n}$ and $\mathbf{P}^{n}(\mathbb{R})$ we have $q_{1}=0, q_{2}=n-1$; for the space $\mathbf{P}^{n}(\mathbb{C}): q_{1}=2 n-2, q_{2}=1$; for the space 
$\mathbf{P}^{n}(\mathbb{H}): q_{1}=4 n-4, q_{2}=3$; and for the space $\mathbf{P}^{2}(\mathbb{C} a): q_{1}=8, q_{2}=7$. Conversely, for the spaces $\mathbf{S}^{n}$ and $\mathbf{P}^{n}(\mathbb{R})$ we could reckon that $q_{1}=n-1, q_{2}=0$. Our choice corresponds to the isometries $\mathbf{P}^{1}(\mathbb{C}) \cong \mathbf{S}^{2}, \mathbf{P}^{1}(\mathbb{H}) \cong \mathbf{S}^{4}$.

Remark 1. Noncompact two-point homogeneous spaces of types 7,8,9,10 are analogous to the compact two-point homogeneous spaces of types 2,4,5,6, respectively. In particular, it means that Lie algebras $\mathfrak{g}$ of symmetry groups of analogous spaces are different real forms of a simple complex Lie algebra. The transition from one such real form to another can be done by multiplying the subspace $\mathfrak{p}$ from the decomposition $\mathfrak{g}=\mathfrak{k} \oplus \mathfrak{p}$ by the imaginary unit $\mathbf{i}$ (or by $-\mathbf{i})$.

The information concerning the representation theory of symmetry groups of two-point homogeneous spaces can be found in 14 .

\section{Invariant differential operators on $M_{\mathbb{S}}$}

Here we shall specify the construction from section 2 for the space $M_{\mathbb{S}}$, where $M$ is a two-point homogeneous compact Riemannian space, using Proposition 1

Let $G$ be the identity component of the isometry group for $M$ and $K$ be its stationary subgroup, corresponding to the point $x_{0} \in M$. The group $G$ naturally acts on the space $M_{\mathbb{S}}$ and this action is transitive 10] (lemma 8.12.1). In particular $K$ acts transitively on the unit sphere $\mathbb{S}_{x_{0}} \subset T_{x_{0}} M$. Identify the space $\mathfrak{p}$ from Proposition $\square$ with the space $T_{x_{0}} M$. After this identification the action of $K$ on $T_{x_{0}} M$ becomes the adjoint action $\operatorname{Ad}_{K}$ on $\mathfrak{p}$. Let $K_{0}$ be the subgroup of $K$, corresponding to the subalgebra $\mathfrak{k}_{0} \subset \mathfrak{k}$. Due to relations (4), (15) $K_{0}$ is the stationary subgroup of the group $G$, corresponding to the point $^{1} y:=\left(x_{0}, \Lambda^{\prime}\right) \in M_{\mathbb{S}}$, where $\Lambda^{\prime}:=\frac{1}{R} \Lambda$.

Let $\tilde{\mathfrak{p}}:=\mathfrak{a} \oplus \mathfrak{p}_{\lambda} \oplus \mathfrak{p}_{2 \lambda} \oplus \mathfrak{k}_{\lambda} \oplus \mathfrak{k}_{2 \lambda}$. Since $\left[\mathfrak{k}_{0}, \tilde{\mathfrak{p}}\right] \subset \tilde{\mathfrak{p}}$ the expansion $\mathfrak{g}=\tilde{\mathfrak{p}} \oplus \mathfrak{k}_{0}$ is reductive. One has $T_{y} M_{\mathbb{S}}=T_{x_{0}} M \oplus T_{\Lambda^{\prime}} \mathbb{S}_{x_{0}}$. Due to Proposition 1 we obtain $\Lambda^{\prime} \perp\left(\mathfrak{p}_{\lambda} \oplus \mathfrak{p}_{2 \lambda}\right)$ and $\left[f_{\lambda, i}, \Lambda^{\prime}\right]=-(2 R)^{-1} e_{\lambda, i}, i=1, \ldots, q_{1},\left[f_{2 \lambda, j}, \Lambda^{\prime}\right]=-R^{-1} e_{2 \lambda, j}, j=1, \ldots, q_{2}$. Therefore the space $\mathfrak{k}_{\lambda} \oplus \mathfrak{k}_{2 \lambda}$ is identified through the $K$-action on $T_{x_{0}} M$ with the space $T_{\Lambda^{\prime}} \mathbb{S}_{x_{0}}$ and the $K_{0}$-action on the space $T_{y} M_{\mathbb{S}} \simeq \tilde{\mathfrak{p}}=\mathfrak{a} \oplus \mathfrak{p}_{\lambda} \oplus \mathfrak{p}_{2 \lambda} \oplus \mathfrak{k}_{\lambda} \oplus \mathfrak{k}_{2 \lambda}$ is again adjoint.

From Proposition 1 we see that $\operatorname{Ad}_{K_{0}}$ conserves all summand in the last expansion. On the other hand the $K_{0}$-action on $T_{\Lambda^{\prime}} \mathbb{S}_{x_{0}}$ is the differential of $K_{0}$-action on $\left(\Lambda^{\prime}\right)^{\perp} \subset T_{x_{0}} M$. Since the last action is linear we obtain that the $\operatorname{Ad}_{K_{0}}$-action in $\mathfrak{p}_{\lambda}$ is equivalent to it's action in $\mathfrak{k}_{\lambda}$ and the $\operatorname{Ad}_{K_{0}}$-action in $\mathfrak{p}_{2 \lambda}$ is equivalent to it's action in $\mathfrak{k}_{2 \lambda}$. Let $\chi_{\lambda}: \mathfrak{k}_{\lambda} \rightarrow$ $\mathfrak{p}_{\lambda}, \chi_{2 \lambda}: \mathfrak{k}_{2 \lambda} \rightarrow \mathfrak{p}_{2 \lambda}$ be isomorphisms of linear spaces such that $\left.\operatorname{Ad}_{K_{0}}\right|_{\mathfrak{p}_{\lambda}} \circ \chi_{\lambda}=\left.\chi_{\lambda} \circ \operatorname{Ad}_{K_{0}}\right|_{\mathfrak{k}_{\lambda}}$ and $\left.\operatorname{Ad}_{K_{0}}\right|_{\mathfrak{p}_{2 \lambda}} \circ \chi_{2 \lambda}=\left.\chi_{2 \lambda} \circ \operatorname{Ad}_{K_{0}}\right|_{\mathfrak{k}_{2 \lambda}}$.

After the substitution $\mathfrak{p} \rightarrow \tilde{\mathfrak{p}}, \mathfrak{k} \rightarrow \mathfrak{k}_{0}$ we can apply the construction from section 2 for calculating generators and relations for the algebra $\operatorname{Diff}\left(M_{\mathbb{S}}\right)$. Let $g_{i}$ are independent invariants of the $\operatorname{Ad}_{K_{0}}$-action in $\tilde{\mathfrak{p}}$. Then elements $\eta \circ \mu\left(g_{i}\right)$ generate the algebra $\operatorname{Diff}\left(M_{\mathbb{S}}\right)$.

There are some obvious invariants of the $\operatorname{Ad}_{K_{0}}$-action in $\tilde{\mathfrak{p}}$. First of all it is $\Lambda \in \mathfrak{a}$, since $\left[\mathfrak{k}_{0}, \mathfrak{a}\right]=0$. Secondly the $\operatorname{Ad}_{K_{0}}$-action is isometric w.r.t. the Killing form which is proportional to the scalar product $\langle\cdot, \cdot\rangle$, so the $\operatorname{Ad}_{K_{0}}$-action conserves the restrictions of $\langle\cdot, \cdot\rangle$ onto spaces $\mathfrak{p}_{\lambda}, \mathfrak{p}_{2 \lambda}, \mathfrak{k}_{\lambda}, \mathfrak{k}_{2 \lambda}$. Thirdly the $\operatorname{Ad}_{K_{0}}$-action conserves functions

$\left\langle\chi_{\lambda} X_{1}, Y_{1}\right\rangle, X_{1} \in \mathfrak{k}_{\lambda}, Y_{1} \in \mathfrak{p}_{\lambda}$ and $\left\langle\chi_{2 \lambda} X_{2}, Y_{2}\right\rangle, X_{2} \in \mathfrak{k}_{2 \lambda}, Y_{2} \in \mathfrak{p}_{2 \lambda}$.

Let define $\chi_{\lambda}$ and $\chi_{2 \lambda}$ by

$$
\chi_{\lambda} X=2[\Lambda, X], X \in \mathfrak{k}_{\lambda}, \chi_{2 \lambda} Y=[\Lambda, Y], Y \in \mathfrak{k}_{2 \lambda} .
$$

It is obvious that $\chi_{\lambda}: \mathfrak{k}_{\lambda} \mapsto \mathfrak{p}_{\lambda}$ and $\chi_{2 \lambda}: \mathfrak{k}_{2 \lambda} \mapsto \mathfrak{p}_{2 \lambda}$ are bijections. For any $k \in K_{0}$ it holds $\operatorname{Ad}_{k} \Lambda=\Lambda$ since $\left[\mathfrak{k}_{0}, \mathfrak{a}\right]=0$, therefore from (6) we obtain: $\operatorname{Ad}_{k} \circ \chi_{\lambda} X=2\left[\Lambda, \operatorname{Ad}_{k} X\right]=$

\footnotetext{
${ }^{1} \mathrm{By}(\alpha, \ldots, \omega)$ we denote the set of objects $\alpha, \ldots, \omega$.
} 
$\chi_{\lambda} \circ \operatorname{Ad}_{k} X, X \in \mathfrak{k}_{\lambda}$ and $\operatorname{Ad}_{k} \circ \chi_{2 \lambda} Y=\left[\Lambda, \operatorname{Ad}_{k} Y\right]=\chi_{2 \lambda} \circ \operatorname{Ad}_{k} Y, Y \in \mathfrak{k}_{2 \lambda}$. It is clear that $\chi_{\lambda} f_{\lambda, i}=e_{\lambda, i}, i=1, \ldots, q_{1}$ and $\chi_{2 \lambda} f_{2 \lambda, j}=e_{2 \lambda, j}, j=1, \ldots, q_{2}$.

The bases

$$
\left\{\frac{1}{R} e_{\lambda, i}\right\}_{i=1}^{q_{1}},\left\{\frac{1}{R} f_{\lambda, i}\right\}_{i=1}^{q_{1}},\left\{\frac{1}{R} e_{2 \lambda, j}\right\}_{j=1}^{q_{2}},\left\{\frac{1}{R} f_{2 \lambda, j}\right\}_{j=1}^{q_{2}}
$$

respectively in spaces $\mathfrak{p}_{\lambda}, \mathfrak{k}_{\lambda}, \mathfrak{p}_{2 \lambda}, \mathfrak{k}_{2 \lambda}$ are orthonormal, so one has the following generators of $\operatorname{Diff}\left(M_{\mathbb{S}}\right)$ :

$$
\begin{aligned}
& D_{0}=\eta(\Lambda), D_{1}=\eta\left(\sum_{i=1}^{q_{1}} e_{\lambda, i}^{2}\right), D_{2}=\eta\left(\sum_{i=1}^{q_{1}} f_{\lambda, i}^{2}\right), D_{3}=\eta\left(\frac{1}{2} \sum_{i=1}^{q_{1}}\left\{e_{\lambda, i}, f_{\lambda, i}\right\}\right), \\
& D_{4}=\eta\left(\sum_{j=1}^{q_{2}} e_{2 \lambda, j}^{2}\right), D_{5}=\eta\left(\sum_{j=1}^{q_{2}} f_{2 \lambda, j}^{2}\right), D_{6}=\eta\left(\frac{1}{2} \sum_{j=1}^{q_{2}}\left\{e_{2 \lambda, j}, f_{2 \lambda, j}\right\}\right),
\end{aligned}
$$

where $\{\cdot, \cdot\}$ means anticommutator. For brevity wee shall omit the symbol $\eta$ below. From (5) one easily obtains:

$$
\begin{aligned}
& {\left[D_{0}, D_{1}\right]=-D_{3},\left[D_{0}, D_{2}\right]=D_{3},\left[D_{0}, D_{3}\right]=\frac{1}{2}\left(D_{1}-D_{2}\right),} \\
& {\left[D_{0}, D_{4}\right]=-2 D_{6},\left[D_{0}, D_{5}\right]=2 D_{6},\left[D_{0}, D_{6}\right]=D_{4}-D_{5} .}
\end{aligned}
$$

In order to find full system of invariants and relations in $\operatorname{Diff}\left(M_{\mathbb{S}}\right)$ we need more detailed information about the $\mathrm{Ad}_{K_{0}}$-action in $\tilde{\mathfrak{p}}$ and commutators in $\mathfrak{g}$. This information will be extracted in the following sections from the models of two-point homogeneous compact Riemannian spaces.

It is easily seen that every automorphism of Lie algebra $\mathfrak{g}$, conserving its subalgebra $\mathfrak{k}_{0}$, generates an automorphism of $\operatorname{Diff}\left(M_{\mathbb{S}}\right)$. From relations (4) one obtains that the map $\sigma: \mathfrak{g} \rightarrow \mathfrak{g},\left.\sigma\right|_{\mathfrak{k}}=\mathrm{id},\left.\sigma\right|_{\mathfrak{p}}=-\mathrm{id}$ is the automorphism of $\mathfrak{g}$. It generates the automorphism of $\operatorname{Diff}\left(M_{\mathbb{S}}\right): D_{0} \rightarrow-D_{0}, D_{1} \rightarrow D_{1}, D_{2} \rightarrow D_{2}, D_{3} \rightarrow-D_{3}, D_{4} \rightarrow D_{4}, D_{5} \rightarrow D_{5}, D_{6} \rightarrow$ $-D_{6}$. We shall denote it by the same symbol $\sigma$.

Another obvious automorphism is the one parametric group $\zeta_{\alpha}$ of internal automorphisms, generated by the $\operatorname{ad}_{\Lambda}$-action. From (5) one obtains:

$$
\begin{aligned}
\zeta_{\alpha}(\Lambda) & =\Lambda, \zeta_{\alpha}\left(e_{\lambda, i}\right)=\cos (\alpha / 2) e_{\lambda, i}-\sin (\alpha / 2) f_{\lambda, i}, \\
\zeta_{\alpha}\left(f_{\lambda, i}\right) & =\sin (\alpha / 2) e_{\lambda, i}+\cos (\alpha / 2) f_{\lambda, i}, i=1, \ldots, q_{1}, \\
\zeta_{\alpha}\left(e_{2 \lambda, j}\right) & =\cos (\alpha) e_{2 \lambda, j}-\sin (\alpha) f_{2 \lambda, j}, \zeta_{\alpha}\left(f_{2 \lambda, i}\right)=\sin (\alpha) e_{2 \lambda, j}+\cos (\alpha) f_{2 \lambda, j}, j=1, \ldots, q_{2} .
\end{aligned}
$$

Therefore

$$
\begin{aligned}
& \zeta_{\alpha}\left(D_{0}\right)=D_{0}, \zeta_{\alpha}\left(D_{1}\right)=\cos ^{2}(\alpha / 2) D_{1}+\sin ^{2}(\alpha / 2) D_{2}-\sin (\alpha) D_{3} \\
& \zeta_{\alpha}\left(D_{2}\right)=\sin ^{2}(\alpha / 2) D_{1}+\cos ^{2}(\alpha / 2) D_{2}+\sin \alpha D_{3} \\
& \zeta_{\alpha}\left(D_{3}\right)=\frac{1}{2} \sin (\alpha)\left(D_{1}-D_{2}\right)+\cos (\alpha) D_{3} \\
& \zeta_{\alpha}\left(D_{4}\right)=\cos ^{2}(\alpha) D_{4}+\sin ^{2}(\alpha) D_{5}-\sin (2 \alpha) D_{6} \\
& \zeta_{\alpha}\left(D_{5}\right)=\sin ^{2}(\alpha) D_{4}+\cos ^{2}(\alpha) D_{5}+\sin (2 \alpha) D_{6} \\
& \zeta_{\alpha}\left(D_{6}\right)=\frac{1}{2} \sin (2 \alpha)\left(D_{4}-D_{5}\right)+\cos (2 \alpha) D_{6}
\end{aligned}
$$

In particular $\zeta_{\pi}\left(D_{1}\right)=D_{2}, \zeta_{\pi}\left(D_{2}\right)=D_{1}, \zeta_{\pi}\left(D_{3}\right)=-D_{3}, \zeta_{\pi}\left(D_{i}\right)=D_{i}, i=0,4,5,6$.

Proposition पimply that the base

$$
\frac{1}{R} \Lambda, \frac{1}{R} e_{\lambda, i}, \frac{1}{R} f_{\lambda, i}, \frac{1}{R} e_{2 \lambda, j}, \frac{1}{R} f_{2 \lambda, j}, i=1, \ldots, q_{1}, j=1, \ldots, q_{2}
$$


in the space $\tilde{\mathfrak{p}}$ is orthonormal, therefore the operator $D^{*}=D_{0}^{2}+D_{1}+D_{2}+D_{4}+D_{5}$ corresponds to the Casimir operator in $U(\mathfrak{g})$ [17] (lecture 18). It means that $D^{*}$ lies in the centre of the algebra $\operatorname{Diff}\left(M_{\mathbb{S}}\right)$.

Let $\pi_{1}: M_{\mathbb{S}} \rightarrow M$ be the canonical projection and $\tilde{\pi}_{1}$ is the map of a function $f$ on $M$ to the function $f \circ \pi_{1}$ on $M_{\mathbb{S}}$. Due to the identification $\mathfrak{p} \simeq T_{x_{0}} M$ it is clear that the operator $\left(D_{0}^{2}+D_{1}+D_{2}\right) \circ \tilde{\pi}_{1}$ is the Laplace-Beltrami operator on $M$.

\section{$5 \quad$ The model for the space $\mathbf{P}^{n}(\mathbb{H})$}

Let $\mathbb{H}$ be the quaternion algebra over the field $\mathbb{R}$ with the base $1, \mathbf{i}, \mathbf{j}, \mathbf{k}$, where $\mathbf{i j}=-\mathbf{j i}=$ $\mathbf{k}, \mathbf{j k}=-\mathbf{k j}=\mathbf{i}, \mathbf{k i}=-\mathbf{i k}=\mathbf{j}$. The conjugation acts as follows: $\overline{x+y \mathbf{i}+z \mathbf{j}+t \mathbf{k}}=$ $x-y \mathbf{i}-z \mathbf{j}-t \mathbf{k}, x, y, z, t \in \mathbb{R}$.

Let $\mathbb{H}^{n+1}$ be the right quaternion space and $\left(z_{1}, \ldots, z_{n+1}\right)$ be coordinates on it. Let $\mathbf{P}^{n}(\mathbb{H})$ be a factor space of the space $\mathbb{H}^{n+1} \backslash\{0\}$ with respect to the right action of the multiplicative group $\mathbb{H}^{*}=\mathbb{H} \backslash\{0\}$. The set $\left(z_{1}: \ldots: z_{n+1}\right)$ up to the multiplication from the right by an arbitrary element from the group $\mathbb{H}^{*}$ is the set of homogeneous coordinates for the element ${ }^{2} \pi(\mathbf{z})$ on the space $\mathbf{P}^{n}(\mathbb{H})$, where $\pi: \mathbb{H}^{n+1} \backslash\{0\} \rightarrow \mathbf{P}^{n}(\mathbb{H})$ is the canonical projection. Let $\langle\mathbf{x}, \mathbf{y}\rangle:=\sum_{i=1}^{n+1} \bar{x}_{i} y_{i}, \mathbf{x}=\left(x_{1}, \ldots, x_{n+1}\right), \mathbf{y}=\left(y_{1}, \ldots, y_{n+1}\right) \in \mathbb{H}^{n+1}$ be the standard scalar product in the space $\mathbb{H}^{n+1}$. Let $\mathbf{z} \in \mathbb{H}^{n+1} \backslash\{0\}, \xi_{i} \in T_{\mathbf{z}} \mathbb{H}^{n+1}, \zeta_{i}=\pi_{*} \xi_{i} \in$ $T_{\pi(\mathbf{z})}\left(\mathbf{P}^{n}(\mathbb{H})\right), i=1,2$. A metric on the space $\mathbf{P}^{n}(\mathbb{H})$

$$
\left.\tilde{g}\right|_{\mathbf{z}}\left(\zeta_{1}, \zeta_{2}\right)=\left(\left\langle\xi_{1}, \xi_{2}\right\rangle\langle\mathbf{z}, \mathbf{z}\rangle-\left\langle\xi_{1}, \mathbf{z}\right\rangle\left\langle\mathbf{z}, \xi_{2}\right\rangle\right) /\langle\mathbf{z}, \mathbf{z}\rangle^{2},
$$

is the analog for the metric with a constant sectional curvature on the space $\mathbf{P}^{n}(\mathbb{R})$ and the metric with a constant holomorphic sectional curvature on the space $\mathbf{P}^{n}(\mathbb{C})$. The real part of the metric (8) is a Riemannian metric on the space $\mathbf{P}^{n}(\mathbb{H})$ :

$$
g=4 R^{2} \operatorname{Re} \tilde{g} .
$$

The normalizing factor in (9) is chosen due to the following reasons. The space $\mathbf{P}^{1}(\mathbb{H})$ with this metric is the sphere $\mathbf{S}^{4}$ with the standard metric of the constant sectional curvature $R^{-2}$. To see this we can consider a homeomorphism $\nu: \mathbf{P}^{1}(\mathbb{H}) \rightarrow \overline{\mathbb{H}} \cong \mathbf{S}^{4}, \nu\left(z_{1}, z_{2}\right)=$ $z_{1}\left(z_{2}\right)^{-1}=z \in \overline{\mathbb{H}}$, where $\overline{\mathbb{H}}$ is the quaternion space completed with the point at infinity. For $n=1$ the formula (9) has the form

$$
g=4 R^{2} \frac{\left(d \bar{z}_{1} d z_{1}+d \bar{z}_{2} d z_{2}\right)\left(\left|z_{1}\right|^{2}+\left|z_{2}\right|^{2}\right)-\left(d \bar{z}_{1} \cdot z_{1}+d \bar{z}_{2} \cdot z_{2}\right)\left(\bar{z}_{1} d z_{1}+\bar{z}_{2} d z_{2}\right)}{\left(\left|z_{1}\right|^{2}+\left|z_{2}\right|^{2}\right)^{2}} .
$$

Using the formula $\left|z_{2}\right|^{2} d z_{1}-z_{1} \bar{z}_{2} d z_{2}=\left|z_{2}\right|^{2}(d z) z_{2}$ by direct calculations we can reduce the expression (10) to the form:

$$
g=\frac{4 R^{2} d z d \bar{z}}{\left(1+|z|^{2}\right)^{2}}
$$

which is the metric with the constant sectional curvature $R^{-2}$ on the sphere $\mathbf{S}^{4}$.

The left action of the group $\mathrm{U}_{\mathbb{H}}(n+1)$, consisting of quaternion matrices $A$ of the size $(n+1) \times(n+1)$ such that $\bar{A}^{T} A=E$, conserves the scalar product $\langle\cdot, \cdot \cdot\rangle$ in the space $\mathbb{H}^{n+1}, \operatorname{dim}_{\mathbb{R}} \mathrm{U}_{\mathbb{H}}(n+1)=(2 n+3)(n+1)$. If we write every quaternion coordinates in $\mathbb{H}^{n+1}$ as a pair of complex numbers, then the group $\mathrm{U}_{\mathbb{H}}(n+1)$ becomes the symplectic group $\operatorname{Sp}(n+1)$.

Left and right multiplications always commute, so the left action of the group $\mathrm{U}_{\mathbb{H}}(n+1)$ is correctly defined also on the space $\mathbf{P}^{n}(\mathbb{H})$. Obviously, it is transitive and conserves

\footnotetext{
${ }^{2}$ To distinguish the point $x \in M$ from their coordinates we shall single out it by the bold type
} 
the metric $g$. The stationary subgroup of the point from the space $\mathbf{P}^{n}(\mathbb{H})$ with the homogeneous coordinates $(1,0, \ldots, 0)$ is the group $\mathrm{U}_{\mathbb{H}}(n) \mathrm{U}_{\mathbb{H}}(1)$, where the group $\mathrm{U}_{\mathbb{H}}(n)$ acts onto the last $n$ coordinates, and the group $\mathrm{U}_{\mathbb{H}}(1)$ acts by the left multiplication of all homogeneous coordinates by quaternions with the unit norm. All stationary subgroups on a homogeneous space are conjugated and hence isomorphic. Therefore $\mathbf{P}^{n}(\mathbb{H})=\mathrm{U}_{\mathbb{H}}(n+$ $1) /\left(\mathrm{U}_{\mathbb{H}}(n) \mathrm{U}_{\mathbb{H}}(1)\right)$.

The Lie algebra $\mathfrak{u}_{\mathbb{H}}(n+1)$ consist of quaternion matrices $A$ of the size $(n+1) \times(n+1)$ such that $\bar{A}^{T}=-A$. Let $E_{k j}$ be the matrix of the size $(n+1) \times(n+1)$ with the unique nonzero element equals 1 , locating at the intersection of the $k$-th row and the $j$-th column. Choose the base for the algebra $\mathfrak{u}_{\mathbb{H}}(n+1)$ as:

$$
\begin{aligned}
& \Psi_{k j}=\frac{1}{2}\left(E_{k j}-E_{j k}\right), 1 \leqslant k<j \leqslant n+1, \Upsilon_{k j}=\frac{\mathbf{i}}{2}\left(E_{k j}+E_{j k}\right), \\
& \Omega_{k j}=\frac{\mathbf{j}}{2}\left(E_{k j}+E_{j k}\right), \Theta_{k j}=\frac{\mathbf{k}}{2}\left(E_{k j}+E_{j k}\right), 1 \leqslant k \leqslant j \leqslant n+1 .
\end{aligned}
$$

The commutative relations for these elements are:

$$
\begin{aligned}
& {\left[\Psi_{k j}, \Psi_{m l}\right]=\frac{1}{2}\left(\delta_{j m} \Psi_{k l}-\delta_{k m} \Psi_{j l}+\delta_{k l} \Psi_{j m}-\delta_{j l} \Psi_{k m}\right),} \\
& {\left[\Psi_{k j}, \Upsilon_{m l}\right]=\frac{1}{2}\left(\delta_{j m} \Upsilon_{k l}-\delta_{k m} \Upsilon_{j l}+\delta_{l j} \Upsilon_{k m}-\delta_{l k} \Upsilon_{j m}\right),} \\
& {\left[\Upsilon_{k j}, \Upsilon_{m l}\right]=\frac{1}{2}\left(\delta_{j m} \Psi_{l k}+\delta_{k m} \Psi_{l j}+\delta_{k l} \Psi_{m j}+\delta_{j l} \Psi_{m k}\right),} \\
& {\left[\Upsilon_{k j}, \Omega_{m l}\right]=\frac{1}{2}\left(\delta_{j m} \Theta_{l k}+\delta_{k m} \Theta_{l j}+\delta_{k l} \Theta_{m j}+\delta_{j l} \Theta_{m k}\right),}
\end{aligned}
$$

plus the analogous equalities, obtaining from the latter three relations by the cyclic permutation $\Upsilon \rightarrow \Omega \rightarrow \Theta \rightarrow \Upsilon$, where $\Psi_{k j}=-\Psi_{j k}, \Psi_{k k}=0, \Upsilon_{k j}=\Upsilon_{j k}, \Omega_{k j}=\Omega_{j k}, \Theta_{k j}=$ $\Theta_{j k}$.

\section{$6 \quad$ Algebras $\operatorname{Diff}\left(\mathbf{P}^{n}(\mathbb{H})_{\mathbb{S}}\right)$ and $\operatorname{Diff}\left(\mathbf{H}^{n}(\mathbb{H})_{\mathbb{S}}\right)$}

Consider now the total space of unit spheres bundle $\mathbf{P}^{n}(\mathbb{H})_{\mathbb{S}}$ over the space $\mathbf{P}^{n}(\mathbb{H})$. Let $(\mathbf{z}, \zeta)$, where $\mathbf{z} \in \mathbf{P}^{n}(\mathbb{H}), \zeta \in T_{\mathbf{z}} \mathbf{P}^{n}(\mathbb{H})$ be a general point of the space $\mathbf{P}^{n}(\mathbb{H})_{\mathbb{S}}$. Due to the isomorphism $\mathbf{P}^{1}(\mathbb{H}) \cong \mathbf{S}^{4}$ we assume here $n \geqslant 2$.

Let $\tilde{\mathbf{z}}_{0}=(1,0, \ldots, 0) \in \mathbb{H}^{n+1}$, an element $\xi_{0} \in T_{\tilde{\mathbf{z}}_{0}} \mathbb{H}^{n+1} \cong \mathbb{H}^{n+1}$ has coordinates $(0,1,0, \ldots, 0)$. Put $\mathbf{z}_{0}=\pi \tilde{\mathbf{z}}_{0}, \zeta_{0}=\pi_{*} \xi_{0} \in T_{\mathbf{z}_{0}} \mathbf{P}^{n}(\mathbb{H})$.

The stationary subgroup $K_{0}$ of the group $\mathrm{U}_{\mathbb{H}}(n+1)$, corresponding to the point $\left(\mathbf{z}_{0}, \zeta_{0}\right)$ is generated by the group $K_{1}=\mathrm{U}_{\mathbb{H}}(n-1)$, acting onto the last $(n-1)$-th homogeneous coordinates and by the group $K_{2}=\mathrm{U}_{\mathbb{H}}(1)$, acting by the left multiplication of all homogeneous coordinates by quaternions with the unit norm. The algebra $\mathfrak{k}_{0}$ of the group $K_{0}$ (corresponding to Proposition 1) is $\left(2 n^{2}-3 n+4\right)$-dimensional and is generated by elements (11) with $3 \leqslant k \leqslant j \leqslant n+1$ and the elements:

$$
\sum_{k=1}^{n+1} \Upsilon_{k k}, \sum_{k=1}^{n+1} \Omega_{k k}, \sum_{k=1}^{n+1} \Theta_{k k} .
$$

Choose the complimentary subspace $\tilde{\mathfrak{p}}$ to the subalgebra $\mathfrak{k}_{0}$ in the algebra $\mathfrak{g}=u_{\mathbb{H}}(n+1)$ as the linear hull of elements:

$$
\begin{gathered}
\Psi_{1 k}, \Upsilon_{1 k}, \Omega_{1 k}, \Theta_{1 k}, 2 \leqslant k \leqslant n+1, \Psi_{2 k}, \Upsilon_{2 k}, \Omega_{2 k}, \Theta_{2 k}, 3 \leqslant k \leqslant n+1, \\
\Upsilon_{*}=\frac{\mathbf{i}}{2}\left(E_{11}-E_{22}\right), \Omega_{*}=\frac{\mathbf{j}}{2}\left(E_{11}-E_{22}\right), \Theta_{*}=\frac{\mathbf{k}}{2}\left(E_{11}-E_{22}\right) .
\end{gathered}
$$


Taking into account relations (12) it is easily obtained that the expansion $\mathfrak{u}_{\mathbb{H}}(n+1)=\tilde{\mathfrak{p}} \oplus \mathfrak{k}_{0}$ is reductive, i.e. $\left[\tilde{\mathfrak{p}}, \mathfrak{k}_{0}\right] \subset \tilde{\mathfrak{p}}$.

It is readily seen from (12) that setting:

$$
\begin{aligned}
\Lambda & =-\Psi_{12}, e_{\lambda, k-2}=\Psi_{1 k}, e_{\lambda, n-3+k}=\Upsilon_{1 k}, e_{\lambda, 2 n-4+k}=\Omega_{1 k}, e_{\lambda, 3 n-5+k}=\Theta_{1 k}, \\
f_{\lambda, k-2} & =-\Psi_{2 k}, f_{\lambda, n-3+k}=-\Upsilon_{2 k}, f_{\lambda, 2 n-4+k}=-\Omega_{2 k}, f_{\lambda, 3 n-5+k}=-\Theta_{2 k}, k=3, \ldots, n+1, \\
e_{2 \lambda, 1} & =\Upsilon_{12}, e_{2 \lambda, 2}=\Omega_{12}, e_{2 \lambda, 3}=\Theta_{12}, f_{2 \lambda, 1}=\Upsilon_{*}, f_{2 \lambda, 2}=\Omega_{*}, f_{2 \lambda, 3}=\Theta_{*},
\end{aligned}
$$

we obtain the base from Proposition 1 for $q_{1}=4 n-4, q_{2}=3$.

Now we are to find the full set of independent $\operatorname{Ad}_{K_{0}}$-invariants in $S(\tilde{\mathfrak{p}})$. According to section 4 the expansion $\tilde{\mathfrak{p}}=\mathfrak{a} \oplus \mathfrak{k}_{\lambda} \oplus \mathfrak{k}_{2 \lambda} \oplus \mathfrak{p}_{\lambda} \oplus \mathfrak{p}_{2 \lambda}$ is invariant w.r.t. the $\operatorname{Ad}_{K_{0}}$-action. In the space $\mathfrak{a}$ the $K_{0}$-action is trivial that gives the invariant $D_{0}=\Lambda \in \mu\left(\mathfrak{p}^{K_{0}}\right)$, already found in section 4

From formulas (14) we see that the space $\mathfrak{p}_{\lambda} \cong \mathbb{H}^{n-1}$ consists of matrices of the form

$$
\left(\begin{array}{cc}
0 & -a^{*} \\
a & 0
\end{array}\right) \equiv\left(\begin{array}{ccccc}
0 & 0 & -\bar{a}_{1} & \ldots & -\bar{a}_{n-1} \\
0 & 0 & 0 & \ldots & 0 \\
a_{1} & 0 & 0 & \ldots & 0 \\
\vdots & \vdots & \vdots & \ddots & \vdots \\
a_{n-1} & 0 & 0 & \ldots & 0
\end{array}\right), a_{1}, \ldots, a_{n-1} \in \mathbb{H} .
$$

Likewise, the space $\mathfrak{k}_{\lambda} \cong \mathbb{H}^{n-1}$ consist of matrices of the form

$$
\left(\begin{array}{ccc}
0 & 0 & 0 \\
0 & 0 & -b^{*} \\
0 & b & 0
\end{array}\right) \equiv\left(\begin{array}{ccccc}
0 & 0 & 0 & \cdots & 0 \\
0 & 0 & -\bar{b}_{1} & \cdots & -\bar{b}_{n-1} \\
0 & b_{1} & 0 & \cdots & 0 \\
\vdots & \vdots & \vdots & \ddots & \vdots \\
0 & b_{n-1} & 0 & \cdots & 0
\end{array}\right), b_{1}, \ldots, b_{n-1} \in \mathbb{H} .
$$

Due to the formula

$$
\left(\begin{array}{cc}
1 & 0 \\
0 & U
\end{array}\right)\left(\begin{array}{cc}
0 & -a^{*} \\
a & 0
\end{array}\right)\left(\begin{array}{cc}
1 & 0 \\
0 & U^{*}
\end{array}\right)=\left(\begin{array}{cc}
0 & -(U a)^{*} \\
U a & 0
\end{array}\right), U \in \mathrm{U}_{\mathbb{H}}(n-1), a \in \mathbb{H}^{n-1}
$$

the action of the group $K_{1}$ in the space $\mathfrak{p}_{\lambda}$ is equivalent to the standard action of the group $U_{\mathbb{H}}(n-1)$ in the space $\mathbb{H}^{n-1}: a \rightarrow U a$. In the space $\mathfrak{k}_{\lambda}$ the action of $K_{1}$ is similar: $b \rightarrow U b$.

The standard action of the group $\mathrm{U}_{\mathbb{H}}(n-1)$ in the space $\mathbb{H}^{n-1}$ has one independent real invariant: $\langle\mathbf{z}, \mathbf{z}\rangle, \mathbf{z} \in \mathbb{H}^{n-1}$, and the diagonal action of $\mathrm{U}_{\mathbb{H}}(n-1)$ in the space $\mathfrak{p}_{\lambda} \oplus \mathfrak{k}_{\lambda} \cong$ $\mathbb{H}^{n-1} \oplus \mathbb{H}^{n-1}$ has six (independent iff $n \geqslant 3$ ) real invariants:

$$
\left\langle\mathbf{z}_{1}, \mathbf{z}_{1}\right\rangle \in \mathbb{R},\left\langle\mathbf{z}_{2}, \mathbf{z}_{2}\right\rangle \in \mathbb{R},\left\langle\mathbf{z}_{1}, \mathbf{z}_{2}\right\rangle \in \mathbb{H} \cong \mathbb{R}^{4}, \mathbf{z}_{1}, \mathbf{z}_{2} \in \mathbb{H}^{n-1} .
$$

Denote the corresponding elements from $\mu\left(\tilde{\mathfrak{p}}^{K_{1}}\right) \in U(\mathfrak{g})^{K_{1}}$ in the following way:

$$
\begin{aligned}
& D_{1}=\sum_{k=3}^{n+1}\left(\Psi_{1 k}^{2}+\Upsilon_{1 k}^{2}+\Omega_{1 k}^{2}+\Theta_{1 k}^{2}\right), D_{2}=\sum_{k=3}^{n+1}\left(\Psi_{2 k}^{2}+\Upsilon_{2 k}^{2}+\Omega_{2 k}^{2}+\Theta_{2 k}^{2}\right), \\
& D_{3}=-\frac{1}{2} \sum_{k=3}^{n+1}\left(\left\{\Psi_{1 k}, \Psi_{2 k}\right\}+\left\{\Upsilon_{1 k}, \Upsilon_{2 k}\right\}+\left\{\Omega_{1 k}, \Omega_{2 k}\right\}+\left\{\Theta_{1 k}, \Theta_{2 k}\right\}\right), \\
& \square_{1}=\frac{1}{2} \sum_{k=3}^{n+1}\left(-\left\{\Psi_{1 k}, \Upsilon_{2 k}\right\}+\left\{\Psi_{2 k}, \Upsilon_{1 k}\right\}+\left\{\Theta_{1 k}, \Omega_{2 k}\right\}-\left\{\Theta_{2 k}, \Omega_{1 k}\right\}\right),
\end{aligned}
$$




$$
\begin{aligned}
& \square_{2}=\frac{1}{2} \sum_{k=3}^{n+1}\left(-\left\{\Psi_{1 k}, \Omega_{2 k}\right\}+\left\{\Psi_{2 k}, \Omega_{1 k}\right\}+\left\{\Upsilon_{1 k}, \Theta_{2 k}\right\}-\left\{\Upsilon_{2 k}, \Theta_{1 k}\right\}\right), \\
& \square_{3}=\frac{1}{2} \sum_{k=3}^{n+1}\left(-\left\{\Psi_{1 k}, \Theta_{2 k}\right\}+\left\{\Psi_{2 k}, \Theta_{1 k}\right\}+\left\{\Omega_{1 k}, \Upsilon_{2 k}\right\}-\left\{\Omega_{2 k}, \Upsilon_{1 k}\right\}\right) .
\end{aligned}
$$

If $n=2$, then there is the unique independent relation between invariants (15):

$$
\left|\left\langle\mathbf{z}_{1}, \mathbf{z}_{2}\right\rangle\right|^{2}=\left|\bar{z}_{1} z_{2}\right|^{2}=\left|z_{1}\right|^{2}\left|z_{2}\right|^{2}=\left\langle\mathbf{z}_{1}, \mathbf{z}_{1}\right\rangle\left\langle\mathbf{z}_{2}, \mathbf{z}_{2}\right\rangle, \mathbf{z}_{1}=z_{1}, \mathbf{z}_{2}=z_{2} \in \mathbb{H} .
$$

If we write this identity in coordinates, then we will obtain the well known Euler identity which is the key ingredient in the proof of the Lagrange theorem from number theory: if two integers have the form $a^{2}+b^{2}+c^{2}+d^{2}, a, b, c, d \in \mathbb{Z}$, then their product has the same form.

The elements $D_{1}, D_{2}, D_{3}$, already found in section 4 are invariant w.r.t. the action of the whole group $K_{0}$, therefore they correspond to operators of the second order from $\operatorname{Diff}\left(\mathbf{P}^{n}(\mathbb{H})_{\mathbb{S}}\right)$. The elements $\square_{1}, \square_{2}, \square_{3}$ are not invariant w.r.t. the action of the group $K_{2} \cong \mathrm{U}_{\mathbb{H}}(1)$. Obviously, the $K_{2}$-action on the linear hull of elements $\square_{1}, \square_{2}, \square_{3}$ is equivalent to the well known action of the group $\mathrm{SO}(3) \cong \mathrm{U}_{\mathbb{H}}(1) /(1,-1)$ in the space $\mathbb{H}^{\prime}$ of pure imaginary quaternions:

$$
x \rightarrow q x \bar{q}, x \in \mathbb{H}^{\prime}, q \in \mathrm{U}_{\mathbb{H}}(1),
$$

after the identification $\square_{1} \leftrightarrow \mathbf{i}, \square_{1} \leftrightarrow \mathbf{j}, \square_{3} \leftrightarrow \mathbf{k}$.

The $K_{2}$-action on 3 -dimensional spaces $\mathfrak{p}_{2 \lambda}, \mathfrak{k}_{2 \lambda}$ are the same after the identification $\Upsilon_{12}, \Upsilon_{*} \leftrightarrow \mathbf{i} ; \Omega_{12}, \Omega_{*} \leftrightarrow \mathbf{j} ; \Theta_{12}, \Theta_{*} \leftrightarrow \mathbf{k}$; while the $K_{1}$-action in these spaces is trivial. Thus we are to find invariants of diagonal action of the group $\mathrm{SO}(3)$ in the space $\mathbb{R}^{3} \oplus$ $\mathbb{R}^{3} \oplus \mathbb{R}^{3}$. It is clear that there are $6=9-3$ such independent invariants:

$$
\langle\mathbf{x}, \mathbf{x}\rangle,\langle\mathbf{y}, \mathbf{y}\rangle,\langle\mathbf{z}, \mathbf{z}\rangle,\langle\mathbf{x}, \mathbf{y}\rangle,\langle\mathbf{x}, \mathbf{z}\rangle,\langle\mathbf{z}, \mathbf{y}\rangle, \mathbf{x}, \mathbf{y}, \mathbf{z} \in \mathbb{R}^{3}
$$

and invariant $\langle\mathbf{x}, \mathbf{y}, \mathbf{z}\rangle \equiv\langle\mathbf{x}, \mathbf{y} \times \mathbf{z}\rangle$ algebraically connected with the first six:

$$
\begin{aligned}
\langle\mathbf{x}, \mathbf{y}, \mathbf{z}\rangle^{2} & =\mathbf{x}^{2} \mathbf{y}^{2} \mathbf{z}^{2}+2\langle\mathbf{x}, \mathbf{y}\rangle\langle\mathbf{x}, \mathbf{z}\rangle\langle\mathbf{y}, \mathbf{z}\rangle-\mathbf{x}^{2}\langle\mathbf{y}, \mathbf{z}\rangle^{2} \\
& -\mathbf{y}^{2}\langle\mathbf{x}, \mathbf{z}\rangle^{2}-\mathbf{z}^{2}\langle\mathbf{x}, \mathbf{y}\rangle^{2}
\end{aligned}
$$

where $\mathbf{y} \times \mathbf{z}$ is the standard vector product in $\mathbb{R}^{3}$. Relation (18) can be verified using the well known formulas: $\langle\mathbf{x}, \mathbf{y}\rangle^{2}=\mathbf{x}^{2} \mathbf{y}^{2}-\langle\mathbf{x} \times \mathbf{y}\rangle^{2}$ and $\mathbf{x} \times(\mathbf{y} \times \mathbf{x})=\langle\mathbf{x}, \mathbf{z}\rangle \mathbf{y}-\langle\mathbf{x}, \mathbf{y}\rangle \mathbf{z}$.

It gives the following invariants from $U(\mathfrak{g})^{K_{0}}$ :

$$
\begin{aligned}
D_{4} & =\Upsilon_{12}^{2}+\Omega_{12}^{2}+\Theta_{12}^{2}, D_{5}=\Upsilon_{*}^{2}+\Omega_{*}^{2}+\Theta_{*}^{2}, \\
D_{6} & =\frac{1}{2}\left(\left\{\Upsilon_{12}, \Upsilon_{*}\right\}+\left\{\Omega_{12}, \Omega_{*}\right\}+\left\{\Theta_{12}, \Theta_{*}\right\}\right), \\
D_{7} & =\frac{1}{2}\left(\left\{\square_{1}, \Upsilon_{12}\right\}+\left\{\square_{2}, \Omega_{12}\right\}+\left\{\square_{3}, \Theta_{12}\right\}\right), \\
D_{8} & =\frac{1}{2}\left(\left\{\square_{1}, \Upsilon_{*}\right\}+\left\{\square_{2}, \Omega_{*}\right\}+\left\{\square_{3}, \Theta_{*}\right\}\right), D_{9}=\square_{1}^{2}+\square_{2}^{2}+\square_{3}^{2}, \\
D_{10} & =\square_{1} \Omega_{12} \Theta_{*}-\square_{1} \Omega_{*} \Theta_{12}+\square_{2} \Upsilon_{*} \Theta_{12}-\square_{2} \Upsilon_{12} \Theta_{*}+\square_{3} \Omega_{*} \Upsilon_{12}-\square_{3} \Omega_{12} \Upsilon_{*} .
\end{aligned}
$$

Here we took into account that every three factors from all summand in the last expression pairwise commutate. The invariants $D_{4}, D_{5}, D_{6}$ correspond to the general case, considered in section 4

In fact invariants $D_{7}, D_{8}, D_{9}$ and $D_{10}$ are not in $\mu\left(\mathfrak{p}^{K_{0}}\right)$ because they are not symmetric w.r.t. all transposition of their factors of the first degree. After complete symmetrization 
we can obtain invariants from $\mu\left(\mathfrak{p}^{K_{0}}\right): \tilde{D}_{k} \equiv D_{k}+D_{k}^{*} \bmod (U(\mathfrak{g}) \mathfrak{k})^{K_{0}}, k=7,8,9,10$, where $D_{k}^{*}$ are elements from $U(\mathfrak{g})^{K_{0}}$ with $\operatorname{deg} D_{k}^{*}<\operatorname{deg} D_{k}$. For convenience we will use elements $D_{k}$ instead of $\tilde{D}_{k}, k=7,8,9,10$.

Thus operators $D_{0}, \ldots, D_{10}$ generate the algebra $\operatorname{Diff}\left(\mathbf{P}^{n}(\mathbb{H})_{\mathbb{S}}\right)$.

The degrees of the generators are as follows:

$$
\begin{aligned}
& \operatorname{deg}\left(D_{0}\right)=1, \operatorname{deg}\left(D_{1}\right)=\operatorname{deg}\left(D_{2}\right)=\operatorname{deg}\left(D_{3}\right)=\operatorname{deg}\left(D_{4}\right)=\operatorname{deg}\left(D_{5}\right)=\operatorname{deg}\left(D_{6}\right)=2, \\
& \operatorname{deg}\left(D_{7}\right)=\operatorname{deg}\left(D_{8}\right)=3, \operatorname{deg}\left(D_{9}\right)=\operatorname{deg}\left(D_{10}\right)=4 .
\end{aligned}
$$

In the model of the space $\mathbf{P}^{n}(\mathbb{H})$ we can transpose the coordinates $z_{1}$ and $z_{2}$. The operators $D_{3}, D_{4}, D_{5}, D_{8}, D_{9}, D_{10}$ are symmetric (invariant) w.r.t. this transposition and the operators $D_{0}, \square_{1}, \square_{2}, \square_{3}, D_{6}, D_{7}$ are skew symmetric. The operators $D_{1}$ and $D_{2}$ turn into each other under this transposition.

It is easily verified that automorphisms $\zeta_{\alpha}, \sigma$ acts on $\square_{i}, D_{7}, \ldots, D_{10}, i=1,2,3$ as

$$
\begin{aligned}
\zeta_{\alpha}\left(\square_{i}\right) & =\square_{i}, i=1,2,3, \zeta_{\alpha}\left(D_{7}\right)=\cos (\alpha) D_{7}-\sin (\alpha) D_{8}, \zeta_{\alpha}\left(D_{8}\right)=\sin (\alpha) D_{7}+\cos (\alpha) D_{8}, \\
\zeta_{\alpha}\left(D_{9}\right) & =D_{9}, \zeta_{\alpha}\left(D_{10}\right)=D_{10}, \sigma\left(\square_{i}\right)=-\square_{i}, i=1,2,3, \sigma\left(D_{7}\right)=D_{7}, \sigma\left(D_{8}\right)=-D_{8}, \\
\sigma\left(D_{9}\right) & =D_{9}, \sigma\left(D_{10}\right)=D_{10} .
\end{aligned}
$$

Taking into account their action on other generators (see section 4) we obtain that the transposition of $z_{1}$ and $z_{2}$ is equivalent to the composition $\sigma \circ \zeta_{\pi}$.

In order to get the generators of the algebra $\operatorname{Diff}\left(\mathbf{H}^{n}(\mathbb{H})_{\mathbb{S}}\right)$ one can use Remark 1 formula (14) and make the formal substitution:

$$
\begin{aligned}
\Lambda & \rightarrow \mathbf{i} \Lambda, \Psi_{1 k} \rightarrow \mathbf{i} \Psi_{1 k}, \Upsilon_{1 k} \rightarrow \mathbf{i} \Upsilon_{1 k}, \Omega_{1 k} \rightarrow \mathbf{i} \Omega_{1 k}, \Theta_{1 k} \rightarrow \mathbf{i} \Theta_{1 k}, \Upsilon_{12} \rightarrow \mathbf{i} \Upsilon_{12}, \\
\Omega_{12} & \rightarrow \mathbf{i} \Omega_{12}, \Theta_{12} \rightarrow \mathbf{i} \Theta_{12}, \Psi_{2 k} \rightarrow \Psi_{2 k}, \Upsilon_{2 k} \rightarrow \Upsilon_{2 k}, \Omega_{2 k} \rightarrow \Omega_{2 k}, \Theta_{2 k} \rightarrow \Theta_{2 k}, \\
\Upsilon_{*} & \rightarrow \Upsilon_{*}, \Omega_{*} \rightarrow \Omega_{*}, \Theta_{*} \rightarrow \Theta_{*}, k=3, \ldots, n+1
\end{aligned}
$$

This substitution produces the following substitution for the generators $D_{0}, \ldots, D_{10}$ :

$$
\begin{aligned}
& D_{0} \rightarrow \mathbf{i} \bar{D}_{0}, D_{1} \rightarrow-\bar{D}_{1}, D_{2} \rightarrow \bar{D}_{2}, D_{3} \rightarrow \mathbf{i} \bar{D}_{3}, D_{4} \rightarrow-\bar{D}_{4}, D_{5} \rightarrow \bar{D}_{5}, \\
& D_{6} \rightarrow \mathbf{i} \bar{D}_{6}, D_{7} \rightarrow-\bar{D}_{7}, D_{8} \rightarrow \mathbf{i} \bar{D}_{8}, D_{9} \rightarrow-\bar{D}_{9}, D_{10} \rightarrow-\bar{D}_{10} .
\end{aligned}
$$

The operators $\bar{D}_{0}, \ldots, \bar{D}_{10}$ generate the algebra $\operatorname{Diff}\left(\mathbf{H}^{n}(\mathbb{H})_{\mathbb{S}}\right)$.

\section{$7 \quad$ Relations in algebras $\operatorname{Diff}\left(\mathbf{P}^{n}(\mathbb{H})_{\mathbb{S}}\right)$ and $\operatorname{Diff}\left(\mathbf{H}^{n}(\mathbb{H})_{\mathbb{S}}\right)$}

Here we are to find the independent relations in $\operatorname{Diff}\left(\mathbf{P}^{n}(\mathbb{H})_{\mathbb{S}}\right)$ for it's generators $D_{0}, \ldots$, $D_{10}$. They are of two types (see section 2). First type is commutative relations, because a commutator of two differential operator of orders $m_{1}$ and $m_{2}$ is an operator of an order $m_{3} \leqslant m_{1}+m_{2}-1$. It gives $11(11-1) / 2=55$ relations. If $n \geqslant 3$ due to (18) the second type consists of only one independent relation of the form:

$$
D_{10}^{2}-D_{4} D_{5} D_{9}-2 D_{6} D_{7} D_{8}+D_{9} D_{6}^{2}+D_{4} D_{8}^{2}+D_{5} D_{7}^{2}=D^{\prime},
$$

where $D^{\prime}$ is an operator of an order $\leqslant 7$, which is polynomial in $D_{0}, \ldots, D_{10}$. If $n=2$ the formula (17) gives another independent relation of the form:

$$
\frac{1}{2}\left\{D_{1}, D_{2}\right\}-D_{3}^{2}-D_{9}=D^{\prime \prime},
$$

where $D^{\prime \prime}$ is an operator of an order $\leqslant 3$, polynomial in $D_{0}, \ldots, D_{8}$. The direct calculations gives $D^{\prime \prime}=D_{1}+D_{2}$, therefore in the case $n=2$ we have the additional relations:

$$
\frac{1}{2}\left\{D_{1}, D_{2}\right\}-D_{3}^{2}-D_{9}=D_{1}+D_{2} .
$$


For $n=2$ using this relation we can exclude the element $D_{9}$ from the list of generators.

In principle all relations can be obtained by straightforward calculations in $U(\mathfrak{g})$ modulo $(U(\mathfrak{g}) \mathfrak{k})^{K_{0}}$, but these calculations became too cumbersome to write all of them here. In Appendix A there is an example of deriving some commutative relation. After getting some commutative relations by direct calculations it is possible to get some other ones (see Appendix A) using the Jacobi identity:

$$
\left[D_{i},\left[D_{j}, D_{k}\right]\right]+\left[D_{k},\left[D_{i}, D_{j}\right]\right]+\left[D_{j},\left[D_{k}, D_{i}\right]\right]=0,
$$

which is valid, in particular, in every associative algebra. This identity gives also a tool for checking the commutative relations already found. Below there are all 55 commutative relations in lexicographic order. The relation (22) became too difficult to obtain in a similar way. May be we need a computer algebra calculations to obtain the explicit expression for $D^{\prime}$.

$$
\begin{aligned}
& {\left[D_{0}, D_{1}\right]=-D_{3},\left[D_{0}, D_{2}\right]=D_{3},\left[D_{0}, D_{3}\right]=\frac{1}{2}\left(D_{1}-D_{2}\right),\left[D_{0}, D_{4}\right]=-2 D_{6},} \\
& {\left[D_{0}, D_{5}\right]=2 D_{6},\left[D_{0}, D_{6}\right]=D_{4}-D_{5},\left[D_{0}, D_{7}\right]=-D_{8},\left[D_{0}, D_{8}\right]=D_{7},\left[D_{0}, D_{9}\right]=0,} \\
& {\left[D_{0}, D_{10}\right]=0,\left[D_{1}, D_{2}\right]=-\left\{D_{0}, D_{3}\right\}-2 D_{7},\left[D_{1}, D_{3}\right]=-\frac{1}{2}\left\{D_{0}, D_{1}\right\}+D_{8}+n(n-1) D_{0} \text {, }} \\
& {\left[D_{1}, D_{4}\right]=2 D_{7},\left[D_{1}, D_{5}\right]=0,\left[D_{1}, D_{6}\right]=D_{8},\left[D_{1}, D_{7}\right]=-\frac{1}{2}\left\{D_{3}, D_{6}\right\}-\frac{1}{2}\left\{D_{1}, D_{4}\right\}} \\
& +\frac{3}{8}\left(D_{1}-D_{2}\right)+D_{9}+D_{10}+n(n-1) D_{4},\left[D_{1}, D_{8}\right]=-\frac{1}{2}\left\{D_{3}, D_{5}\right\}-\frac{1}{2}\left\{D_{1}, D_{6}\right\} \\
& +\frac{3}{4} D_{3}+n(n-1) D_{6},\left[D_{1}, D_{9}\right]=-\left\{D_{3}, D_{8}\right\}-\left\{D_{1}, D_{7}\right\}-\frac{3}{4}\left\{D_{0}, D_{3}\right\} \\
& +2\left(n-\frac{3}{2}\right)\left(n+\frac{1}{2}\right) D_{7},\left[D_{1}, D_{10}\right]=\frac{1}{2}\left\{D_{6}, D_{8}\right\}-\frac{1}{2}\left\{D_{5}, D_{7}\right\}+\frac{3}{8}\left\{D_{0}, D_{3}\right\}+\frac{1}{2} D_{7}, \\
& {\left[D_{2}, D_{3}\right]=\frac{1}{2}\left\{D_{0}, D_{2}\right\}+D_{8}-n(n-1) D_{0},\left[D_{2}, D_{4}\right]=-2 D_{7},\left[D_{2}, D_{5}\right]=0,} \\
& {\left[D_{2}, D_{6}\right]=-D_{8},\left[D_{2}, D_{7}\right]=-\frac{1}{2}\left\{D_{3}, D_{6}\right\}+\frac{1}{2}\left\{D_{2}, D_{4}\right\}+\frac{3}{8}\left(D_{1}-D_{2}\right)-D_{9}-D_{10}} \\
& -n(n-1) D_{4},\left[D_{2}, D_{8}\right]=-\frac{1}{2}\left\{D_{3}, D_{5}\right\}+\frac{1}{2}\left\{D_{2}, D_{6}\right\}+\frac{3}{4} D_{3}-n(n-1) D_{6}, \\
& {\left[D_{2}, D_{9}\right]=-\left\{D_{3}, D_{8}\right\}+\left\{D_{2}, D_{7}\right\}+\frac{3}{4}\left\{D_{0}, D_{3}\right\}-2\left(n-\frac{3}{2}\right)\left(n+\frac{1}{2}\right) D_{7},} \\
& {\left[D_{2}, D_{10}\right]=-\frac{1}{2}\left\{D_{6}, D_{8}\right\}+\frac{1}{2}\left\{D_{5}, D_{7}\right\}-\frac{3}{8}\left\{D_{0}, D_{3}\right\}-\frac{1}{2} D_{7},\left[D_{3}, D_{4}\right]=0,\left[D_{3}, D_{5}\right]=2 D_{8} \text {, }} \\
& {\left[D_{3}, D_{6}\right]=D_{7},\left[D_{3}, D_{7}\right]=-\frac{1}{4}\left\{D_{1}+D_{2}, D_{6}\right\}+n(n-1) D_{6},\left[D_{3}, D_{8}\right]=-\frac{1}{4}\left\{D_{1}+D_{2}, D_{5}\right\}} \\
& +n(n-1) D_{5}+D_{9}+D_{10},\left[D_{3}, D_{9}\right]=-\frac{1}{2}\left\{D_{1}+D_{2}, D_{8}\right\}+\frac{3}{8}\left\{D_{0}, D_{1}-D_{2}\right\} \\
& +2\left(n-\frac{3}{2}\right)\left(n+\frac{1}{2}\right) D_{8},\left[D_{3}, D_{10}\right]=\frac{1}{2}\left\{D_{6}, D_{7}\right\}-\frac{1}{2}\left\{D_{4}, D_{8}\right\}-\frac{3}{16}\left\{D_{0}, D_{1}-D_{2}\right\} \\
& +\frac{1}{2} D_{8},\left[D_{4}, D_{5}\right]=-2\left\{D_{0}, D_{6}\right\},\left[D_{4}, D_{6}\right]=-\left\{D_{0}, D_{4}\right\}+\frac{3}{2} D_{0}, \\
& {\left[D_{4}, D_{7}\right]=\frac{1}{2}\left\{D_{1}-D_{2}, D_{4}\right\}+\frac{3}{4}\left(D_{2}-D_{1}\right),\left[D_{4}, D_{8}\right]=\frac{1}{2}\left\{D_{1}-D_{2}, D_{6}\right\}-\left\{D_{0}, D_{7}\right\},} \\
& {\left[D_{4}, D_{9}\right]=\left\{D_{1}-D_{2}, D_{7}\right\},\left[D_{4}, D_{10}\right]=0,\left[D_{5}, D_{6}\right]=\left\{D_{0}, D_{5}\right\}-\frac{3}{2} D_{0},} \\
& {\left[D_{5}, D_{7}\right]=\left\{D_{3}, D_{6}\right\}+\left\{D_{0}, D_{8}\right\},\left[D_{5}, D_{8}\right]=\left\{D_{3}, D_{5}\right\}-\frac{3}{2} D_{3},\left[D_{5}, D_{9}\right]=2\left\{D_{3}, D_{8}\right\},}
\end{aligned}
$$




$$
\begin{aligned}
{\left[D_{5}, D_{10}\right] } & =0,\left[D_{6}, D_{7}\right]=\frac{1}{4}\left\{D_{1}-D_{2}, D_{6}\right\}+\frac{1}{2}\left\{D_{3}, D_{4}\right\}+\frac{1}{2}\left\{D_{0}, D_{7}\right\}-\frac{3}{4} D_{3}, \\
{\left[D_{6}, D_{8}\right] } & =\frac{1}{4}\left\{D_{1}-D_{2}, D_{5}\right\}+\frac{1}{2}\left\{D_{3}, D_{6}\right\}-\frac{1}{2}\left\{D_{0}, D_{8}\right\}+\frac{3}{8}\left(D_{2}-D_{1}\right), \\
{\left[D_{6}, D_{9}\right] } & =\frac{1}{2}\left\{D_{1}-D_{2}, D_{8}\right\}+\left\{D_{3}, D_{7}\right\},\left[D_{6}, D_{10}\right]=0,\left[D_{7}, D_{8}\right]=\frac{1}{4}\left\{D_{1}-D_{2}, D_{8}\right\} \\
& -\frac{1}{2}\left\{D_{3}, D_{7}\right\}+\frac{3}{16}\left\{D_{0}, D_{1}+D_{2}\right\}-\frac{1}{2}\left\{D_{0}, D_{9}+D_{10}\right\}-\frac{3}{4} n(n-1) D_{0}, \\
{\left[D_{7}, D_{9}\right] } & =\frac{1}{4}\left\{D_{3}, D_{6}\right\}+\frac{1}{8}\left\{D_{1}-D_{2}, D_{4}\right\}+\frac{1}{2}\left\{D_{1}-D_{2}, D_{9}+D_{10}\right\}-\frac{3}{8}\left(D_{1}^{2}-D_{2}^{2}\right) \\
& +\frac{3}{4}\left(n^{2}-n-\frac{1}{4}\right)\left(D_{1}-D_{2}\right),\left[D_{7}, D_{10}\right]=\frac{1}{4}\left\{D_{2}-D_{1}, D_{6}^{2}\right\}-\frac{1}{4}\left\{\left\{D_{0}, D_{7}\right\}, D_{6}\right\} \\
& +\frac{1}{4}\left\{\left\{D_{0}, D_{4}\right\}, D_{8}\right\}+\frac{1}{8}\left\{\left\{D_{1}-D_{2}, D_{5}\right\}, D_{4}\right\}-\frac{1}{4}\left\{D_{3}, D_{6}\right\} \\
& +\frac{1}{8}\left\{D_{2}-D_{1}, 3 D_{4}+D_{5}\right\}-\frac{1}{2}\left\{D_{0}, D_{8}\right\}+\frac{15}{32}\left(D_{1}-D_{2}\right), \\
{\left[D_{8}, D_{9}\right] } & =\frac{1}{8}\left\{D_{1}-D_{2}, D_{6}\right\}+\frac{1}{4}\left\{D_{3}, D_{5}\right\}-\frac{3}{8}\left\{D_{3}, D_{1}+D_{2}\right\}+\left\{D_{3}, D_{9}+D_{10}\right\} \\
& +\frac{3}{2}\left(n^{2}-n-\frac{1}{4}\right) D_{3},\left[D_{8}, D_{10}\right]=-\frac{1}{4}\left\{\left\{D_{3}, D_{6}\right\}, D_{6}\right\}+\frac{1}{4}\left\{\left\{D_{0}, D_{6}\right\}, D_{8}\right\} \\
& -\frac{1}{4}\left\{\left\{D_{0}, D_{5}\right\}, D_{7}\right\}+\frac{1}{4}\left\{\left\{D_{3}, D_{5}\right\}, D_{4}\right\}-\frac{1}{2}\left\{D_{3}, D_{5}\right\}-\frac{1}{4}\left\{D_{3}, D_{4}\right\}+\frac{1}{4}\left\{D_{0}, D_{7}\right\} \\
& +\frac{9}{16} D_{3},\left[D_{9}, D_{10}\right]=\frac{1}{4}\left\{-\left\{D_{6}, D_{8}\right\}+\left\{D_{5}, D_{7}\right\}, D_{1}-D_{2}\right\}+\frac{1}{2}\left\{\left\{D_{3}, D_{8}\right\}, D_{4}\right\} \\
& -\frac{1}{2}\left\{\left\{D_{3}, D_{6}\right\}, D_{7}\right\}+\frac{1}{4}\left\{D_{2}-D_{1}, D_{7}\right\}-\frac{1}{2}\left\{D_{3}, D_{8}\right\} .
\end{aligned}
$$

It is interesting that the operators $D_{9}$ and $D_{10}$ arise in the right hand sides of these relations only in the combination $D_{9}+D_{10}$.

Using relations (24) it is not difficult to verify that the operator $D^{*}=D_{0}^{2}+D_{1}+D_{2}+$ $D_{4}+D_{5}$ lies in the centre of the algebra $\operatorname{Diff}\left(\mathbf{P}^{n}(\mathbb{H})_{\mathbb{S}}\right)$ in accordance with the section 4

Using substitution (21) one can obtains from (24) the commutative relations for the algebra $\operatorname{Diff}\left(\mathbf{H}^{n}(\mathbb{H})_{\mathbb{S}}\right)$.

The analog for the operator $D^{*}$ from the centre of the algebra now becomes $\bar{D}^{*}=$ $\bar{D}_{0}^{2}+\bar{D}_{1}-\bar{D}_{2}+\bar{D}_{4}-\bar{D}_{5} \in \operatorname{Diff}\left(\mathbf{H}^{n}(\mathbb{H})_{\mathbb{S}}\right)$. In the case $n=2$ the additional relations (23) becomes:

$$
\frac{1}{2}\left\{\bar{D}_{1}, \bar{D}_{2}\right\}-\bar{D}_{3}^{2}-\bar{D}_{9}=\bar{D}_{1}-\bar{D}_{2} .
$$

\section{$8 \quad$ Algebras $\operatorname{Diff}\left(\mathbf{P}^{n}(\mathbb{C})_{\mathbb{S}}\right)$ and $\operatorname{Diff}\left(\mathbf{H}^{n}(\mathbb{C})_{\mathbb{S}}\right)$}

\subsection{The model for the space $\mathrm{P}^{n}(\mathbb{C})$}

Taking the factor space of $\mathbb{C}^{n+1} \backslash\{0\}$ w.r.t the action of the multiplicative group $\mathbb{C}^{*}=$ $\mathbb{C} \backslash\{0\}$ (due to the commutativity of the complex multiplication it makes no difference left or right), we obtain the complex projective space $\mathbf{P}^{n}(\mathbb{C})$. Let $\pi: \mathbb{C}^{n+1} \backslash\{0\} \rightarrow \mathbf{P}^{n}(\mathbb{C})$ be the canonical projection. Let now $\langle\mathbf{x}, \mathbf{y}\rangle:=\sum_{i=1}^{n+1} \bar{x}_{i} y_{i}, \mathbf{x}=\left(x_{1}, \ldots, x_{n+1}\right), \mathbf{y}=\left(y_{1}, \ldots, y_{n+1}\right) \in$ $\mathbb{C}^{n+1}$ be the standard scalar product in the space $\mathbb{C}^{n+1}$.

The metric $\tilde{g}$ of the constant holomorphic sectional curvature on the space $\mathbf{P}^{n}(\mathbb{C})$ is defined by the same formula (8) as on the space $\mathbf{P}^{n}(\mathbb{H})$, where now $\mathbf{z} \in \mathbb{C}^{n+1} \backslash\{0\}, \xi_{i} \in$ $T_{\mathbf{z}} \mathbb{C}^{n+1}, \zeta_{i}=\pi_{*} \xi_{i} \in T_{\pi(\mathbf{z})}\left(\mathbf{P}^{n}(\mathbb{C})\right), i=1,2$. 
The Riemannian metric $g$ on the space $\mathbf{P}^{n}(\mathbb{C})$ is:

$$
g=4 R^{2} \operatorname{Re} \tilde{g} .
$$

If $n=2$ it is not difficult to verify (like in section 5 ) that the homeomorphism $\tau: \mathbf{P}^{1}(\mathbb{C}) \rightarrow$ $\overline{\mathbb{C}} \cong \mathbf{S}^{2}, \tau\left(z_{1}, z_{2}\right)=z_{1}\left(z_{2}\right)^{-1}=z \in \overline{\mathbb{C}}$, transforms (25) into the metric

$$
g=\frac{4 R^{2} d z d \bar{z}}{\left(1+|z|^{2}\right)^{2}}
$$

of the sectional curvature $R^{-2}$ on the sphere $\mathbf{S}^{2}$.

The left action of the group $G=\mathrm{SU}(n+1)$ on the space $\mathbb{C}^{n+1}$ conserves the scalar product $\langle\cdot, \cdot\rangle$ and induces the action in the space $\mathbf{P}^{n}(\mathbb{C})$, conserving metrics $\tilde{g}$ and $g$.

The stationary subgroup, corresponding to the point of the space $\mathbf{P}^{n}(\mathbb{C})$ with homogeneous coordinates $(1: 0: \ldots: 0)$, are the group $\mathrm{U}(n)=\mathrm{SU}(n) \mathrm{U}(1)$, where the factor $\mathrm{SU}(n)$ acts in the standard way onto the last $n$ coordinates, and the factor $\mathrm{U}(1)$ acts by the multiplication of the first coordinate by $e^{\mathbf{i} \phi}$ and the second one by $e^{-\mathbf{i} \phi}, \phi \in R \bmod 2 \pi$. Thus $\mathbf{P}^{n}(\mathbb{C})=\mathrm{SU}(n+1) / \mathrm{U}(n)$.

Choose a base of the algebra $\mathfrak{s u}(n+1)$ in the form:

$$
\begin{gathered}
\Psi_{k j}=\frac{1}{2}\left(E_{k j}-E_{j k}\right), \Upsilon_{k j}=\frac{\mathbf{i}}{2}\left(E_{k j}+E_{j k}\right), 1 \leqslant k<j \leqslant n+1, \\
\Upsilon_{k}=\frac{\mathbf{i}}{2}\left(E_{11}-E_{k k}\right), 2 \leqslant k \leqslant n+1 .
\end{gathered}
$$

The commutators for these elements are easily extracted from (12), taking into account that $\Upsilon_{k}=\frac{1}{2}\left(\Upsilon_{11}-\Upsilon_{k k}\right)$ using the notations from (11).

\subsection{Algebras $\operatorname{Diff}\left(\mathbf{P}^{n}(\mathbb{C})_{\mathbb{S}}\right)$ and $\operatorname{Diff}\left(\mathbf{H}^{n}(\mathbb{C})_{\mathbb{S}}\right)$}

Consider now the space $\mathbf{P}^{n}(\mathbb{C})_{\mathbb{S}}$. Due to the isomorphism $\mathbf{P}^{1}(\mathbb{C}) \cong \mathbf{S}^{2}$ we again assume that $n \geqslant 2$.

Let $\tilde{\mathbf{z}}_{0}=(1,0, \ldots, 0) \in \mathbb{C}^{n+1}$, an element $\xi_{0} \in T_{\tilde{\mathbf{z}}_{0}} \mathbb{C}^{n+1} \cong \mathbb{C}^{n+1}$ has coordinates $(0,1,0, \ldots, 0)$. Put $\mathbf{z}_{0}=\pi \tilde{\mathbf{z}}_{0}, \zeta_{0}=\pi_{*} \xi_{0} \in T_{\mathbf{z}_{0}} \mathbf{P}^{n}(\mathbb{C})$.

The stationary subgroup $K_{0}$ of the group $\mathrm{SU}(n+1)$, corresponding to the point $\left(\mathbf{z}_{0}, \zeta_{0}\right)$, is generated by the group $K_{1}=\mathrm{SU}(n-1)$, acting onto the last $n-1$-th coordinates and by the group $K_{2}=\mathrm{U}(1)$, acting onto the homogeneous coordinates of $\mathbf{P}^{n}(\mathbb{C})$ as:

$$
\left(x_{1}: \ldots: x_{n+1}\right) \rightarrow\left(e^{\mathbf{i} \phi} x_{1}: e^{\mathbf{i} \phi} x_{2}: e^{-2 \mathbf{i} \phi} x_{3}: x_{4}: \ldots: x_{n+1}\right),
$$

$\operatorname{dim}_{\mathbb{R}} K_{0}=(n-1)^{2}$ and we obtain $K_{0} \cong \mathrm{U}(n-1)$.

The algebra $\mathfrak{k}_{0}$ of the group $K_{0}$ is the linear hull of elements (26) as $3 \leqslant k<j \leqslant n+1$ and elements:

$$
\Upsilon_{j}-\Upsilon_{3}=\frac{\mathbf{i}}{2}\left(E_{33}-E_{j j}\right), 3<j \leqslant n+1,2 \Upsilon_{3}-\Upsilon_{2}=\frac{\mathbf{i}}{2}\left(E_{11}+E_{22}-2 E_{33}\right) .
$$

Choose the complimentary subspace $\tilde{\mathfrak{p}}$ to the subalgebra $\mathfrak{k}_{0}$ in the algebra $\mathfrak{g}=\mathfrak{s u}(n+1)$ as the linear hull of elements:

$$
\Psi_{1 k}, \Upsilon_{1 k}, 2 \leqslant k \leqslant n+1, \Psi_{2 k}, \Upsilon_{2 k}, 3 \leqslant k \leqslant n+1, \Upsilon_{*}=\Upsilon_{2}
$$

Taking into account relations (12) it is easily obtained that the expansion $\mathfrak{s u}(n+1)=\tilde{\mathfrak{p}} \oplus \mathfrak{k}_{0}$ is reductive, i.e. $\left[\tilde{\mathfrak{p}}, \mathfrak{k}_{0}\right] \subset \tilde{\mathfrak{p}}$. 
We will obtain the particular case of Proposition 1 for $q_{1}=2 n-2, q_{2}=1$ setting:

$$
\begin{aligned}
\Lambda=-\Psi_{12}, e_{\lambda, k-2} & =\Psi_{1 k}, e_{\lambda, n-3+k}=\Upsilon_{1 k}, f_{\lambda, k-2}=-\Psi_{2 k}, f_{\lambda, n-3+k}=-\Upsilon_{2 k}, \\
e_{2 \lambda, 1} & =\Upsilon_{12}, f_{2 \lambda, 1}=\Upsilon_{*}, k=3, \ldots, n+1 .
\end{aligned}
$$

Now we are to find the generators of $\operatorname{Ad}_{K_{0}}$-invariants in $S(\tilde{\mathfrak{p}})$. The expansion $\tilde{\mathfrak{p}}=$ $\mathfrak{a} \oplus \mathfrak{k}_{\lambda} \oplus \mathfrak{k}_{2 \lambda} \oplus \mathfrak{p}_{\lambda} \oplus \mathfrak{p}_{2 \lambda}$ is invariant w.r.t. the $\operatorname{Ad}_{K_{0}}$-action. In the spaces $\mathfrak{a}, \mathfrak{p}_{2 \lambda}, \mathfrak{k}_{2 \lambda}$ the $K_{0}$-action is trivial that gives the invariants $D_{0}=\Lambda, D_{4}=\Upsilon_{12}, D_{5}=\Upsilon_{*} \in \mu\left(\mathfrak{p}^{K_{0}}\right)$. Operators $D_{4}, D_{5}$ are square roots of their analogs from section 4

From formulas (30) we see that the space $\mathfrak{p}_{\lambda} \cong \mathbb{C}^{n-1}$ consists of matrices of the form

$$
\left(\begin{array}{cc}
0 & -a^{*} \\
a & 0
\end{array}\right) \equiv\left(\begin{array}{ccccc}
0 & 0 & -\bar{a}_{1} & \ldots & -\bar{a}_{n-1} \\
0 & 0 & 0 & \ldots & 0 \\
a_{1} & 0 & 0 & \cdots & 0 \\
\vdots & \vdots & \vdots & \ddots & \vdots \\
a_{n-1} & 0 & 0 & \cdots & 0
\end{array}\right), a_{1}, \ldots, a_{n-1} \in \mathbb{C} .
$$

Similarly, the space $\mathfrak{k}_{\lambda} \cong \mathbb{C}^{n-1}$ consist of matrices of the form

$$
\left(\begin{array}{ccc}
0 & 0 & 0 \\
0 & 0 & -b^{*} \\
0 & b & 0
\end{array}\right) \equiv\left(\begin{array}{ccccc}
0 & 0 & 0 & \ldots & 0 \\
0 & 0 & -\bar{b}_{1} & \cdots & -\bar{b}_{n-1} \\
0 & b_{1} & 0 & \cdots & 0 \\
\vdots & \vdots & \vdots & \ddots & \vdots \\
0 & b_{n-1} & 0 & \cdots & 0
\end{array}\right), b_{1}, \ldots, b_{n-1} \in \mathbb{C} .
$$

The action of the group $K_{1}$ in the spaces $\mathfrak{p}_{\lambda}$ and $\mathfrak{k}_{\lambda}$ is equivalent to the standard action of the group $\mathrm{SU}(n-1)$ in the space $\mathbb{C}^{n-1}: a \rightarrow U a, U \in \mathrm{SU}(n-1)$, likewise in section [6 It is easy to verify that the action (28) generates the action $a_{1} \rightarrow \exp ^{-3 \mathbf{i} \phi} a_{1}, a_{i} \rightarrow$ $\exp ^{-\mathbf{i} \phi} a_{i}, b_{1} \rightarrow \exp ^{-3 \mathbf{i} \phi} b_{1}, b_{i} \rightarrow \exp ^{-\mathbf{i} \phi} b_{i}, i=2, \ldots, n-1$. Therefore the $K_{0}$-action in spaces $\mathfrak{p}_{\lambda}$ and $\mathfrak{k}_{\lambda}$ is equivalent to the standard $\mathrm{U}(n-1)$-action in $\mathbb{C}^{n-1}$.

This action has one independent real invariant: $\langle\mathbf{z}, \mathbf{z}\rangle, \mathbf{z} \in \mathbb{C}^{n-1}$, and the diagonal action of $\mathrm{U}(n-1)$ in the space $\mathfrak{p}_{\lambda} \oplus \mathfrak{k}_{\lambda} \cong \mathbb{C}^{n-1} \oplus \mathbb{C}^{n-1}$ has four (independent iff $n \geqslant 3$ ) real invariants:

$$
\left\langle\mathbf{z}_{1}, \mathbf{z}_{1}\right\rangle \in \mathbb{R},\left\langle\mathbf{z}_{2}, \mathbf{z}_{2}\right\rangle \in \mathbb{R},\left\langle\mathbf{z}_{1}, \mathbf{z}_{2}\right\rangle \in \mathbb{C} \cong \mathbb{R}^{2}, \mathbf{z}_{1}, \mathbf{z}_{2} \in \mathbb{C}^{n-1} .
$$

Denote the corresponding elements from $\mu\left(\tilde{\mathfrak{p}}^{K_{0}}\right) \in U(\mathfrak{g})^{K_{0}}$ in the following way:

$$
\begin{aligned}
& D_{1}=\sum_{k=3}^{n+1}\left(\Psi_{1 k}^{2}+\Upsilon_{1 k}^{2}\right), D_{2}=\sum_{k=3}^{n+1}\left(\Psi_{2 k}^{2}+\Upsilon_{2 k}^{2}\right) \\
& D_{3}=-\frac{1}{2} \sum_{k=3}^{n+1}\left(\left\{\Psi_{1 k}, \Psi_{2 k}\right\}+\left\{\Upsilon_{1 k}, \Upsilon_{2 k}\right\}\right), \square=\frac{1}{2} \sum_{k=3}^{n+1}\left(-\left\{\Psi_{1 k}, \Upsilon_{2 k}\right\}+\left\{\Psi_{2 k}, \Upsilon_{1 k}\right\}\right) .
\end{aligned}
$$

In this case only operator $\square$ is new w.r.t. section 4

If $n=2$, then there is the unique independent relation between invariants (31):

$$
\left|\left\langle\mathbf{z}_{1}, \mathbf{z}_{2}\right\rangle\right|^{2}=\left|\bar{z}_{1} z_{2}\right|^{2}=\left|z_{1}\right|^{2}\left|z_{2}\right|^{2}=\left\langle\mathbf{z}_{1}, \mathbf{z}_{1}\right\rangle\left\langle\mathbf{z}_{2}, \mathbf{z}_{2}\right\rangle, \mathbf{z}_{1}=z_{1}, \mathbf{z}_{2}=z_{2} \in \mathbb{C} .
$$

Thus operators $D_{0}, \ldots, D_{5}, \square$ generate the algebra $\operatorname{Diff}\left(\mathbf{P}^{n}(\mathbb{C})_{\mathbb{S}}\right)$.

The degrees of the generators are as follows:

$$
\operatorname{deg}\left(D_{0}\right)=\operatorname{deg}\left(D_{4}\right)=\operatorname{deg}\left(D_{5}\right)=1, \operatorname{deg}\left(D_{1}\right)=\operatorname{deg}\left(D_{2}\right)=\operatorname{deg}\left(D_{3}\right)=\operatorname{deg}(\square)=2 .
$$


The operators $D_{3}, D_{4}$ are symmetric and the operators $D_{0}, \square, D_{5}$ are skew symmetric w.r.t. the transposition of coordinates $z_{1}$ and $z_{2}$. The operators $D_{1}$ and $D_{2}$ turn into each other under this transposition.

In order to get the generators of the algebra $\operatorname{Diff}\left(\mathbf{H}^{n}(\mathbb{C})_{\mathbb{S}}\right)$ we can use the formal substitution:

$$
\begin{aligned}
\Lambda & \rightarrow \mathbf{i} \Lambda, \Psi_{1 k} \rightarrow \mathbf{i} \Psi_{1 k}, \Upsilon_{1 k} \rightarrow \mathbf{i} \Upsilon_{1 k}, \Upsilon_{12} \rightarrow \mathbf{i} \Upsilon_{12} \\
\Psi_{2 k} & \rightarrow \Psi_{2 k}, \Upsilon_{2 k} \rightarrow \Upsilon_{2 k}, \Upsilon_{*} \rightarrow \Upsilon_{*}, k=3, \ldots, n+1
\end{aligned}
$$

This substitution produces the following substitution for the generators $D_{0}, \ldots, D_{5}, \square$ :

$$
D_{0} \rightarrow \mathbf{i} \bar{D}_{0}, D_{1} \rightarrow-\bar{D}_{1}, D_{2} \rightarrow \bar{D}_{2}, D_{3} \rightarrow \mathbf{i} \bar{D}_{3}, D_{4} \rightarrow \mathbf{i} \bar{D}_{4}, \square \rightarrow \mathbf{i} \bar{\square}, D_{5} \rightarrow \bar{D}_{5}
$$

The operators $\bar{D}_{0}, \ldots, \bar{D}_{5}, \bar{\square}$ generate the algebra $\operatorname{Diff}\left(\mathbf{H}^{n}(\mathbb{C})_{\mathbb{S}}\right)$.

\subsection{Relations in algebras $\operatorname{Diff}\left(\mathbf{P}^{n}(\mathbb{C})_{\mathbb{S}}\right)$ and $\operatorname{Diff}\left(\mathbf{H}^{n}(\mathbb{C})_{\mathbb{S}}\right)$}

The commutative relation for the algebra $\operatorname{Diff}\left(\mathbf{P}^{n}(\mathbb{C})_{\mathbb{S}}\right)$ are as follows:

$$
\begin{aligned}
& {\left[D_{0}, D_{1}\right]=-D_{3},\left[D_{0}, D_{2}\right]=D_{3},\left[D_{0}, D_{3}\right]=\frac{1}{2}\left(D_{1}-D_{2}\right),\left[D_{0}, D_{4}\right]=-D_{5},\left[D_{0}, D_{5}\right]=D_{4},} \\
& {\left[D_{0}, \square\right]=0,\left[D_{1}, D_{2}\right]=-\left\{D_{0}, D_{3}\right\}-\left\{\square, D_{4}\right\},\left[D_{1}, D_{3}\right]=-\frac{1}{2}\left\{D_{0}, D_{1}\right\}+\frac{1}{2}\left\{\square, D_{5}\right\}} \\
& +\frac{(n-1)^{2}}{4} D_{0},\left[D_{1}, D_{4}\right]=\square,\left[D_{1}, D_{5}\right]=0,\left[D_{1}, \square\right]=-\frac{1}{2}\left\{D_{1}, D_{4}\right\}-\frac{1}{2}\left\{D_{3}, D_{5}\right\} \\
& +\frac{(n-1)^{2}}{4} D_{4},\left[D_{2}, D_{3}\right]=\frac{1}{2}\left\{D_{0}, D_{2}\right\}+\frac{1}{2}\left\{\square, D_{5}\right\}-\frac{(n-1)^{2}}{4} D_{0},\left[D_{1}, D_{4}\right]=\square, \\
& {\left[D_{2}, D_{5}\right]=0,\left[D_{2}, \square\right]=\frac{1}{2}\left\{D_{2}, D_{4}\right\}-\frac{1}{2}\left\{D_{3}, D_{5}\right\}-\frac{(n-1)^{2}}{4} D_{4},\left[D_{3}, D_{4}\right]=0,} \\
& {\left[D_{3}, D_{5}\right]=\square,\left[D_{3}, \square\right]=-\frac{1}{4}\left\{D_{1}+D_{2}, D_{5}\right\}+\frac{(n-1)^{2}}{4} D_{5},\left[D_{4}, D_{5}\right]=-D_{0},} \\
& {\left[D_{4}, \square\right]=\frac{1}{2}\left(D_{1}-D_{2}\right),\left[D_{5}, \square\right]=D_{3} .}
\end{aligned}
$$

If $n>2$ then there are no relations of the second type. If $n=2$ then there is one relation of the second type due to (33):

$$
\frac{1}{2}\left\{D_{1}, D_{2}\right\}-D_{3}^{2}-\square^{2}-\frac{1}{4}\left(D_{0}^{2}+D_{4}^{2}+D_{5}^{2}\right)=0 .
$$

It is easy to verify that the operator $D^{*}=D_{0}^{2}+D_{1}+D_{2}+D_{4}^{2}+D_{5}^{2}$ lies in the centre of the algebra $\operatorname{Diff}\left(\mathbf{P}^{n}(\mathbb{C})_{\mathbb{S}}\right)$ in accordance with the section 4

Using substitution (35) we obtain analogous relations for the algebra $\operatorname{Diff}\left(\mathbf{H}^{n}(\mathbb{C})_{\mathbb{S}}\right)$.

The commutative relation are now as follows:

$$
\begin{aligned}
& {\left[\bar{D}_{0}, \bar{D}_{1}\right]=\bar{D}_{3},\left[\bar{D}_{0}, \bar{D}_{2}\right]=\bar{D}_{3},\left[\bar{D}_{0}, \bar{D}_{3}\right]=\frac{1}{2}\left(\bar{D}_{2}+\bar{D}_{1}\right),\left[\bar{D}_{0}, \bar{D}_{4}\right]=\bar{D}_{5},\left[\bar{D}_{0}, \bar{D}_{5}\right]=\bar{D}_{4},} \\
& {\left[\bar{D}_{0}, \bar{\square}\right]=0,\left[\bar{D}_{1}, \bar{D}_{2}\right]=-\left\{\bar{D}_{0}, \bar{D}_{3}\right\}-\left\{\bar{\square}, \bar{D}_{4}\right\},\left[\bar{D}_{1}, \bar{D}_{3}\right]=-\frac{1}{2}\left\{\bar{D}_{0}, \bar{D}_{1}\right\}-\frac{1}{2}\left\{\bar{\square}, \bar{D}_{5}\right\}} \\
& -\frac{(n-1)^{2}}{4} \bar{D}_{0},\left[\bar{D}_{1}, \bar{D}_{4}\right]=-\bar{\square},\left[\bar{D}_{1}, \bar{D}_{5}\right]=0,\left[\bar{D}_{1}, \bar{\square}\right]=-\frac{1}{2}\left\{\bar{D}_{1}, \bar{D}_{4}\right\}+\frac{1}{2}\left\{\bar{D}_{3}, \bar{D}_{5}\right\} \\
& -\frac{(n-1)^{2}}{4} \bar{D}_{4},\left[\bar{D}_{2}, \bar{D}_{3}\right]=\frac{1}{2}\left\{\bar{D}_{0}, \bar{D}_{2}\right\}+\frac{1}{2}\left\{\bar{\square}, \bar{D}_{5}\right\}-\frac{(n-1)^{2}}{4} \bar{D}_{0},\left[\bar{D}_{1}, \bar{D}_{4}\right]=-\bar{\square}, \\
& {\left[\bar{D}_{2}, \bar{D}_{5}\right]=0,\left[\bar{D}_{2}, \bar{\square}\right]=\frac{1}{2}\left\{\bar{D}_{2}, \bar{D}_{4}\right\}-\frac{1}{2}\left\{\bar{D}_{3}, \bar{D}_{5}\right\}-\frac{(n-1)^{2}}{4} \bar{D}_{4},\left[\bar{D}_{3}, \bar{D}_{4}\right]=0,}
\end{aligned}
$$




$$
\begin{aligned}
& {\left[\bar{D}_{3}, \bar{D}_{5}\right]=\bar{\square},\left[\bar{D}_{3}, \bar{\square}\right]=-\frac{1}{4}\left\{\bar{D}_{1}-\bar{D}_{2}, \bar{D}_{5}\right\}-\frac{(n-1)^{2}}{4} \bar{D}_{5},\left[\bar{D}_{4}, \bar{D}_{5}\right]=-\bar{D}_{0},} \\
& {\left[\bar{D}_{4}, \bar{\square}\right]=\frac{1}{2}\left(\bar{D}_{1}+\bar{D}_{2}\right),\left[\bar{D}_{5}, \bar{\square}\right]=\bar{D}_{3} .}
\end{aligned}
$$

If $n>2$ then there are no relations of the second type. If $n=2$ then there is one relation of the second type analogous to (36):

$$
\frac{1}{2}\left\{\bar{D}_{1}, \bar{D}_{2}\right\}-\bar{D}_{3}^{2}-\bar{\square}^{2}-\frac{1}{4}\left(\bar{D}_{0}^{2}+\bar{D}_{4}^{2}-\bar{D}_{5}^{2}\right)=0 .
$$

The operator $\bar{D}^{*}=\bar{D}_{0}^{2}+\bar{D}_{1}-\bar{D}_{2}+\bar{D}_{4}^{2}-\bar{D}_{5}^{2}$ lies in the centre of the algebra $\operatorname{Diff}\left(\mathbf{H}^{n}(\mathbb{C})_{\mathbb{S}}\right)$.

\section{$9 \quad$ Algebras $\operatorname{Diff}\left(\mathbf{P}^{n}(\mathbb{R})_{\mathbb{S}}\right), \operatorname{Diff}\left(\mathbf{S}_{\mathbb{S}}^{n}\right)$ and $\operatorname{Diff}\left(\mathbf{H}^{n}(\mathbb{R})_{\mathbb{S}}\right)$}

\subsection{Generators of algebras $\operatorname{Diff}\left(\mathbf{S}_{\mathbb{S}}^{n}\right)$ and $\operatorname{Diff}\left(\mathbf{H}^{n}(\mathbb{R})_{\mathbb{S}}\right)$}

Let now $\langle\cdot, \cdot\rangle$ be the standard scalar product in the space $\mathbb{R}^{n+1}$. The equation $\langle\mathbf{x}, \mathbf{x}\rangle=R>0$ defines the sphere $\mathbf{S}^{n} \cong \mathrm{SO}(n+1) / \mathrm{SO}(n) \subset \mathbb{R}^{n+1}$ of the radius $R$ with the induced metric on it. The space $\mathbf{P}^{n}(\mathbb{R})$ is the factor space of $\mathbf{S}^{n}$ w.r.t. the relation: $\mathbf{x} \sim-\mathbf{x}$. Below we will show that algebras $\operatorname{Diff}\left(\mathbf{P}^{n}(\mathbb{R})_{\mathbb{S}}\right)$ and $\operatorname{Diff}\left(\mathbf{S}_{\mathbb{S}}^{n}\right)$ are isomorphic.

The spaces $\mathbf{S}_{\mathbb{S}}^{1}, \mathbf{P}^{1}(\mathbb{R})_{\mathbb{S}}$ are one dimensional and the algebra of invariant differential operators on them is generated by one differential operator of the first order. Therefore we again suppose that $n \geq 2$.

Let

$$
\Psi_{k j}=\frac{1}{2}\left(E_{k j}-E_{j k}\right), 1 \leqslant k<j \leqslant n+1
$$

be the base of the algebra $\mathfrak{s o}(n+1)$. The commutative relations for them are contained in (12).

Consider the space $\mathbf{S}_{\mathbb{S}}^{n}$. Let $\tilde{\mathbf{z}}_{0}=(1,0, \ldots, 0) \in \mathbb{R}^{n+1}$, an element $\xi_{0} \in T_{\tilde{\mathbf{z}}_{0}} \mathbb{R}^{n+1} \cong \mathbb{R}^{n+1}$ has coordinates $(0,1,0, \ldots, 0)$. Put $\mathbf{z}_{0}=\pi \tilde{\mathbf{z}}_{0}, \zeta_{0}=\pi_{*} \xi_{0} \in T_{\mathbf{z}_{0}} \mathbf{S}_{\mathbb{S}}^{n}$.

The stationary subgroup $K_{0}$ of the group $\mathrm{SO}(n+1)$, corresponding to the point $\left(\mathbf{z}_{0}, \zeta_{0}\right) \in \mathbf{S}_{\mathbb{S}}^{n}$, is the group $\mathrm{SO}(n-1)$, acting onto the last $(n-1)$-th coordinates.

The group $\mathrm{SO}(n+1)$ is a group covering of the identity component $G$ of the isometry group for $\mathbf{P}^{n}(\mathbb{R})$. The group $K_{0}=\mathrm{SO}(n-1) \subset \mathrm{SO}(n+1)$ is a group covering of its analog $K_{0}^{\prime} \subset G$. The kernel of such covering is a normal subgroup of $\operatorname{SO}(n-1)$ that lies in the centre of $\mathrm{SO}(n-1)$ [17] (lecture 9). Therefore the orbits of $\operatorname{Ad}_{K_{0}}$ and $\operatorname{Ad}_{K_{0}^{\prime}}$ actions on $\mathfrak{p} \subset \mathfrak{g}$ coincide and the construction from section 2 implies the isomorphism $\operatorname{Diff}\left(\mathbf{P}^{n}(\mathbb{R})_{\mathbb{S}}\right) \cong \operatorname{Diff}\left(\mathbf{S}_{\mathbb{S}}^{n}\right)$.

The algebra $\mathfrak{k}_{0}$ of the group $K_{0}$ is the linear hull of elements $\Psi_{k j}$ as $3 \leqslant k<j \leqslant n+1$. Choose the complimentary subspace $\tilde{\mathfrak{p}}$ to the subalgebra $\mathfrak{k}_{0}$ in the algebra $\mathfrak{g}=\mathfrak{s o}(n+1)$ as the linear hull of elements:

$$
\Psi_{1 k}, 2 \leqslant k \leqslant n+1, \Psi_{2 k}, 3 \leqslant k \leqslant n+1 .
$$

The expansion $\mathfrak{s o}(n+1)=\tilde{\mathfrak{p}} \oplus \mathfrak{s o}(n-1)$ is reductive.

We will obtain the particular case of Proposition $\square$ for $q_{1}=0, q_{2}=n-1$ setting:

$$
\Lambda=-2 \Psi_{12}, e_{2 \lambda, k-2}=2 \Psi_{1 k}, f_{2 \lambda, k-2}=-2 \Psi_{2 k}, k=3, \ldots, n+1 .
$$

Now we have the expansion $\tilde{\mathfrak{p}}=\mathfrak{a} \oplus \mathfrak{k}_{2 \lambda} \oplus \mathfrak{p}_{2 \lambda}$, which is invariant w.r.t. the $\operatorname{Ad}_{K_{0}}$-action. It is easy to see that in the space $\mathfrak{a}$ the $K_{0}$-action is trivial and in the spaces $\mathfrak{k}_{2 \lambda}$ and $\mathfrak{p}_{2 \lambda}$ it is equivalent to the standard action of the group $\mathrm{SO}(n-1)$ in the space $\mathbb{R}^{n-1}$. The $K_{0}$-action in the space $\mathfrak{a}$ has the invariant $D_{0}=\Lambda$. The description of base $K_{0}$-invariants in the space $\mathfrak{p}_{2 \lambda} \oplus \mathfrak{k}_{2 \lambda}$ is different in cases $n=2, n=3$ and $n \geqslant 4$. 


\subsubsection{The case $n \geqslant 4$}

The $\mathrm{SO}(n-1)$-action in $\mathbb{R}^{n-1}$ has one independent real invariant: $\langle\mathbf{z}, \mathbf{z}\rangle, \mathbf{z} \in \mathbb{R}^{n-1}$, and the diagonal action of $\mathrm{SO}(n-1)$ in the space $\mathfrak{p}_{2 \lambda} \oplus \mathfrak{k}_{2 \lambda} \cong \mathbb{R}^{n-1} \oplus \mathbb{R}^{n-1}$ has three independent real invariants:

$$
\left\langle\mathbf{z}_{1}, \mathbf{z}_{1}\right\rangle,\left\langle\mathbf{z}_{2}, \mathbf{z}_{2}\right\rangle,\left\langle\mathbf{z}_{1}, \mathbf{z}_{2}\right\rangle, \mathbf{z}_{1}, \mathbf{z}_{2} \in \mathbb{R}^{n-1} .
$$

Denote the corresponding elements from $\mu\left(\tilde{\mathfrak{p}}^{K_{0}}\right) \in U(\mathfrak{g})^{K_{0}}$ in the following way:

$$
D_{1}=4 \sum_{k=3}^{n+1} \Psi_{1 k}^{2}, D_{2}=4 \sum_{k=3}^{n+1} \Psi_{2 k}^{2}, D_{3}=-2 \sum_{k=3}^{n+1}\left\{\Psi_{1 k}, \Psi_{2 k}\right\} \text {. }
$$

All these invariants were found in section 4 for the general situation.

Thus operators $D_{0}, D_{1}, D_{2}, D_{3}$ generate the algebra $\operatorname{Diff}\left(\mathbf{S}_{\mathbb{S}}^{n}\right)$.

The degrees of the generators are as follows:

$$
\operatorname{deg}\left(D_{0}\right)=1, \operatorname{deg}\left(D_{1}\right)=\operatorname{deg}\left(D_{2}\right)=\operatorname{deg}\left(D_{3}\right)=2 .
$$

The operator $D_{3}$ is symmetric and the operators $D_{0}$ is skew symmetric w.r.t. the transposition of coordinates $z_{1}$ and $z_{2}$. The operators $D_{1}$ and $D_{2}$ turn into each other under this transposition.

\subsubsection{The case $n=2$}

In this case $K_{0}$ is the trivial group and the independent invariants are $D_{0}, D_{1}=e_{2 \lambda, 1}, D_{2}=$ $f_{2 \lambda, 1}$. Thus the algebra $\operatorname{Diff}\left(\mathbf{S}_{\mathbb{S}}^{2}\right)$ is isomorphic to $U(\mathfrak{s o}(3))$. The centre of this algebra is generated by the operator $D_{0}^{2}+D_{1}^{2}+D_{2}^{2}$.

\subsubsection{The case $n=3$}

In this case $K_{0}=\mathfrak{s o}(2)$ and we have the additional (with respect to the case $n \geqslant 4$ ) invariant of the second order

$$
\square=2\left(\left\{\Psi_{13}, \Psi_{24}\right\}-\left\{\Psi_{14}, \Psi_{23}\right\}\right) .
$$

It is algebraically connected with operators $D_{0}, D_{1}, D_{2}, D_{3}$ which are defined as in the case $n \geqslant 4$.

\subsubsection{Generators of the algebra $\operatorname{Diff}\left(\mathbf{H}^{n}(\mathbb{R})_{\mathbb{S}}\right)$}

First, let $n \geqslant 4$. In order to get the generators of the algebra Diff $\left(\mathbf{H}^{n}(\mathbb{R})_{\mathbb{S}}\right)$ we can use the formal substitution:

$$
\Lambda \rightarrow \mathbf{i} \Lambda, \Psi_{1 k} \rightarrow \mathbf{i} \Psi_{1 k}, \Psi_{2 k} \rightarrow \Psi_{2 k}, k=3, \ldots, n+1 .
$$

This substitution produces the following substitution for the generators $D_{0}, \ldots, D_{3}$ :

$$
D_{0} \rightarrow \mathbf{i} \bar{D}_{0}, D_{1} \rightarrow-\bar{D}_{1}, D_{2} \rightarrow \bar{D}_{2}, D_{3} \rightarrow \mathbf{i} \bar{D}_{3} .
$$

The operators $\bar{D}_{0}, \ldots, \bar{D}_{3}$ generate the algebra $\operatorname{Diff}\left(\mathbf{H}^{n}(\mathbb{R})_{\mathbb{S}}\right)$.

In the case $n=3$ we have the additional substitution $\square \rightarrow \mathbf{i} \square$ and the operators $\bar{D}_{0}, \ldots, \bar{D}_{3}, \bar{\square}$ generate the algebra $\operatorname{Diff}\left(\mathbf{H}^{3}(\mathbb{R})_{\mathbb{S}}\right)$.

In the case $n=2$ we obtain the substitution

$$
D_{0} \rightarrow \mathbf{i} \bar{D}_{0}, D_{1} \rightarrow \mathbf{i} \bar{D}_{1}, D_{2} \rightarrow \bar{D}_{2} .
$$

The algebra $\operatorname{Diff}\left(\mathbf{H}^{2}(\mathbb{R})_{\mathbb{S}}\right)$ is isomorphic to $U(\mathfrak{s o}(2,1))$ and its centre is generated by the operator $D_{0}^{2}+D_{1}^{2}-D_{2}^{2}$. 


\subsection{Relations in algebras $\operatorname{Diff}\left(\mathbf{S}_{\mathbb{S}}^{n}\right)$ and $\operatorname{Diff}\left(\mathbf{H}^{n}(\mathbb{R})_{\mathbb{S}}\right)$}

Here we shall consider only the case $n \geqslant 3$, since $\operatorname{Diff}\left(\mathbf{S}_{\mathbb{S}}^{2}\right) \cong U(\mathfrak{s o}(3))$ and $\operatorname{Diff}\left(\mathbf{H}^{2}(\mathbb{R})_{\mathbb{S}}\right) \cong$ $U(\mathfrak{s o}(2,1))$.

The commutative relation for the algebra $\operatorname{Diff}\left(\mathbf{S}_{\mathbb{S}}^{n}\right)$ are as follows:

$$
\begin{aligned}
& {\left[D_{0}, D_{1}\right]=-2 D_{3},\left[D_{0}, D_{2}\right]=2 D_{3},\left[D_{0}, D_{3}\right]=D_{1}-D_{2},\left[D_{1}, D_{2}\right]=-2\left\{D_{0}, D_{3}\right\},} \\
& {\left[D_{1}, D_{3}\right]=-\left\{D_{0}, D_{1}\right\}+\frac{(n-1)(n-3)}{2} D_{0},\left[D_{2}, D_{3}\right]=\left\{D_{0}, D_{2}\right\}-\frac{(n-1)(n-3)}{2} D_{0} .}
\end{aligned}
$$

For $n=3$ the additional operator $\square$ lies in the centre of the algebra $\operatorname{Diff}\left(\mathbf{S}_{\mathbb{S}}^{3}\right)$.

If $n>3$ then there are no relations of the second type. If $n=3$ then there is one relation of the second type:

$$
\frac{1}{2}\left\{D_{1}, D_{2}\right\}-D_{0}^{2}=D_{3}^{2}+\square^{2}
$$

It is easy to verify that the operators $D_{1}^{*}=D_{0}^{2}+D_{1}+D_{2}$ and

$$
D_{2}^{*}=\frac{1}{2}\left\{D_{1}, D_{2}\right\}-D_{3}^{2}+\left(1-\frac{(n-3)(n-1)}{4}\right)\left(D_{1}+D_{2}\right)
$$

lie in the centre of the algebra $\operatorname{Diff}\left(\mathbf{S}_{\mathbb{S}}^{n}\right)$. If $n=3$ it holds $D_{2}^{*}=\square^{2}+D_{1}^{*}$ due to (44). In this case two operators $D_{1}^{*}$ and $\square$ generate the centre of the algebra $\operatorname{Diff}\left(\mathbf{S}_{\mathbb{S}}^{3}\right)$.

Using substitution (43) we obtain analogous relations for the algebra $\operatorname{Diff}\left(\mathbf{H}^{n}(\mathbb{R})_{\mathbb{S}}\right)$.

The commutative relation are now as follows:

$$
\begin{aligned}
& {\left[\bar{D}_{0}, \bar{D}_{1}\right]=2 \bar{D}_{3},\left[\bar{D}_{0}, \bar{D}_{2}\right]=2 \bar{D}_{3},\left[\bar{D}_{0}, \bar{D}_{3}\right]=\bar{D}_{2}+\bar{D}_{1},\left[\bar{D}_{1}, \bar{D}_{2}\right]=-2\left\{\bar{D}_{0}, \bar{D}_{3}\right\},} \\
& {\left[\bar{D}_{1}, \bar{D}_{3}\right]=-\left\{\bar{D}_{0}, \bar{D}_{1}\right\}-\frac{(n-1)(n-3)}{2} \bar{D}_{0},\left[\bar{D}_{2}, \bar{D}_{3}\right]=\left\{\bar{D}_{0}, \bar{D}_{2}\right\}-\frac{(n-1)(n-3)}{2} \bar{D}_{0},}
\end{aligned}
$$

and for $n=3$ also

$$
\left[\bar{D}_{0}, \square\right]=\left[\bar{D}_{1}, \square\right]=\left[\bar{D}_{2}, \square\right]=\left[\bar{D}_{3}, \square\right]=0 .
$$

The first three relations were found in [4, but the other relations were not calculated there.

If $n>3$ then there are no relations of the second type. If $n=3$ then there is one relation of the second type analogous to (44):

$$
\frac{1}{2}\left\{\bar{D}_{1}, \bar{D}_{2}\right\}-\bar{D}_{0}^{2}=\bar{D}_{3}^{2}+\bar{\square}^{2} .
$$

The operators $\bar{D}_{1}^{*}=\bar{D}_{0}^{2}+\bar{D}_{1}-\bar{D}_{2}$ and

$$
\bar{D}_{2}^{*}=\frac{1}{2}\left\{\bar{D}_{1}, \bar{D}_{2}\right\}-\bar{D}_{3}^{2}+\left(1-\frac{(n-3)(n-1)}{4}\right)\left(\bar{D}_{1}-\bar{D}_{2}\right)
$$

lie in the centre of the algebra $\operatorname{Diff}\left(\mathbf{H}^{n}(\mathbb{R})_{\mathbb{S}}\right)$ and if $n=3$ it holds $\bar{D}_{2}^{*}=\bar{\square}^{2}+\bar{D}_{1}^{*}$ due to (45). In this case the operators $\bar{D}_{1}^{*}$ and $\bar{\square}$ generate the centre of the algebra $\operatorname{Diff}\left(\mathbf{H}_{\mathbb{S}}^{3}\right)$.

\section{The model of the space $\mathbf{P}^{2}(\mathbb{C} a)$}

Our description of Caley algebra $\mathbb{C} a$ and the octonionic projective plane $\mathbf{P}^{2}(\mathbb{C} a)$ in this section is based on [16, [17, 18]. 


\subsection{The algebra $\mathbb{C} a$}

According to Frobenius theorem there are only four finite dimensional division algebras over $\mathbb{R}: \mathbb{R}$ itself and algebras $\mathbb{C}, \mathbb{H}, \mathbb{C} a$. The latter is an eight dimensional normed division algebra of octonions. It is noncommutative and nonassociative, but alternative, i.e. for any two elements $\xi, \eta \in \mathbb{C} a$ it holds $(\xi \eta) \eta=\xi(\eta \eta)$ and $\xi(\xi \eta)=(\xi \xi) \eta$. The group of all automorphisms of $\mathbb{C} a$ is the exceptional simple compact 14-dimensional Lie group $G_{2}$. The standard base of $\mathbb{C} a$ over $\mathbb{R}$ is $\left\{e_{i}\right\}_{i=0}^{7}$, where $e_{0}=1 \in \mathbb{R}$ and $e_{i}^{2}=-1, e_{i} e_{j}=-e_{j} e_{i}, i, j=$ $1, \ldots, 7, i \neq j$. The elements $\left\{e_{i}\right\}_{i=1}^{7}$ are multiplied according to the following scheme:

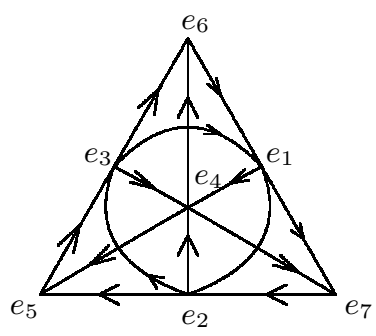

Here $e_{i} e_{j}=e_{k}$ if these elements lie on one line or on the circle and are ordered by arrows as $e_{i}, e_{j}, e_{k}$. The conjugation $\iota: \mathbb{C} a \mapsto \mathbb{C} a$ acts as $\iota\left(e_{0}\right) \equiv \bar{e}_{0}=e_{0}, \iota\left(e_{i}\right) \equiv \bar{e}_{i}=$ $-e_{i}, i=1, \ldots, 7$ and is extended by linearity over whole $\mathbb{C} a$. Let $\operatorname{Re} \xi=\frac{1}{2}(\xi+\bar{\xi}), \operatorname{Im} \xi=$ $\frac{1}{2}(\xi-\bar{\xi}), \xi \in \mathbb{C} a$. Define the scalar product in $\mathbb{C} a$ by the formula: $\langle\eta, \xi\rangle=\frac{1}{2}(\bar{\eta} \xi+\bar{\xi} \eta)=$ $\operatorname{Re}(\bar{\xi} \eta)=\operatorname{Re}(\bar{\eta} \xi) \in \mathbb{R}$ and the norm by the formula $\|\eta\|=\langle\eta, \eta\rangle^{1 / 2}$. In the algebra $\mathbb{C} a$ every two elements generate an associative subalgebra and the following central Moufang identity is valid:

$$
u \cdot x y \cdot u=u x \cdot y u, u, x, y \in \mathbb{C} a .
$$

Here we use the notations $u \cdot x y:=u(x y), x y \cdot u:=(x y) u$.

There are the following description of spinor and vector representations (all 8-dimensional) of the group $\operatorname{Spin}(8)$ in $\mathbb{C} a$ [17, [19, which will be used later. Define linear operators in $\mathbb{C} a$ :

$$
\begin{aligned}
L_{\alpha}: \xi & \mapsto \frac{1}{2} e_{\alpha} \xi, \alpha=1, \ldots, 7, \xi \in \mathbb{C} a, \\
L_{\alpha, \beta}: \xi & \mapsto \frac{1}{2} e_{\alpha}\left(e_{\beta} \xi\right), 1 \leqslant \alpha<\beta \leqslant 7, \xi \in \mathbb{C} a .
\end{aligned}
$$

This operators are generators of the left spinor representation of the group Spin(8), i.e. they are the images of some base of the Lie algebra $\mathfrak{s p i n}(8)$ under this representation. Similarly, operators

$$
\begin{aligned}
R_{\alpha}: \xi & \mapsto \frac{1}{2} \xi e_{\alpha}, \alpha=1, \ldots, 7, \xi \in \mathbb{C} a, \\
R_{\alpha, \beta}: \xi & \mapsto \frac{1}{2}\left(\xi e_{\beta}\right) e_{\alpha}, 1 \leqslant \alpha<\beta \leqslant 7, \xi \in \mathbb{C} a
\end{aligned}
$$

are generators of the right spinor representation of the group Spin(8). All these operators are skew symmetric w.r.t. the scalar product in $\mathbb{C} a$.

Formulae above define operators $L_{\alpha, \beta}, R_{\alpha, \beta}$ also for $1 \leqslant \beta<\alpha \leqslant 7$. If $\mathbb{C} a^{\prime}$ is the space of pure imaginary octonions, $u \in \mathbb{C} a^{\prime}, \xi \in \mathbb{C} a$, then due to the alternativity of $\mathbb{C} a$ :

$$
\xi u \cdot u=\xi u^{2}=-\xi|u|^{2}=-|u|^{2} \xi=u \cdot u \xi .
$$

For $u=e_{\alpha}+e_{\beta}, 1 \leqslant \alpha<\beta \leqslant 7$ it holds

$$
-2 \xi=-\xi\left|e_{\alpha}+e_{\beta}\right|^{2}=\xi\left(e_{\alpha}+e_{\beta}\right) \cdot\left(e_{\alpha}+e_{\beta}\right)=\xi e_{\alpha} \cdot e_{\alpha}+\xi e_{\alpha} \cdot e_{\beta}+\xi e_{\beta} \cdot e_{\alpha}+\xi e_{\beta} \cdot e_{\beta}
$$




$$
=-\xi+\xi e_{\alpha} \cdot e_{\beta}+\xi e_{\beta} \cdot e_{\alpha}-\xi
$$

and $\xi e_{\alpha} \cdot e_{\beta}+\xi e_{\beta} \cdot e_{\alpha}=0$. Similarly, $e_{\alpha} \cdot e_{\beta} \xi+e_{\beta} \cdot e_{\alpha} \xi=0$. For $0 \leqslant i, j \leqslant 7, i \neq j$ we can write more general formulae, useful in the following:

$$
e_{i} \cdot e_{j} \xi=-\bar{e}_{j} \cdot \bar{e}_{i} \xi, \xi e_{i} \cdot e_{j}=-\xi \bar{e}_{j} \cdot \bar{e}_{i}, \xi \in \mathbb{C} a .
$$

In particular, we have $L_{\alpha, \beta}=-L_{\beta, \alpha}, R_{\alpha, \beta}=-R_{\beta, \alpha}, 1 \leqslant \alpha, \beta \leqslant 7, \alpha \neq \beta$.

For the element $g \in \operatorname{Spin}(8)$ denote by $g^{L}, g^{R}$ and $g^{V}$ its images under left spinor, right spinor and vector representation respectively. The following proposition is a version of the triality principle for the group $\operatorname{Spin}(8)^{3}$.

Proposition 2 ([17]). For any element $g \in \operatorname{Spin}(8)$ it holds

$$
g^{V}(\xi \eta)=g^{L}(\xi) \cdot g^{R}(\eta), \xi, \eta \in \mathbb{C} a .
$$

Conversely, if $A, B, C$ are orthogonal operators $\mathbb{C} a \mapsto \mathbb{C} a$ such that

$$
A(\xi \eta)=B(\xi) \cdot C(\eta),
$$

for any $\xi, \eta \in \mathbb{C} a$, then there exist unique $g \in \operatorname{Spin}(8)$ such that $A=g^{V}, B=g^{L}, C=g^{R}$.

From equation (48) we obtain its infinitesimal analogs:

$$
\begin{aligned}
V_{i}(\xi \eta) & =L_{i}(\xi) \cdot \eta+\xi \cdot R_{i}(\eta), i=1, \ldots, 7, \\
V_{i, j}(\xi \eta) & =L_{i, j}(\xi) \cdot \eta+\xi \cdot R_{i, j}(\eta), 1 \leqslant i<j \leqslant 7, \xi, \eta \in \mathbb{C} a,
\end{aligned}
$$

where $V_{i}$ and $V_{i, j}$ are generators of the vector representation of the group $\operatorname{Spin}(8)$.

\subsection{The Jordan algebra $\mathfrak{h}_{3}(\mathbb{C} a)$}

The Hermitian conjugation $A \mapsto A^{*}$ for square matrix with octonion entries is defined as the composition of octonionic conjugation and transposition of $A$, similar to complex or quaternion cases. A matrix $A$ is called Hermitian iff $A^{*}=A$. The simple exceptional Jordan algebra $\mathfrak{h}_{3}(\mathbb{C} a)$ consists of all Hermitian $3 \times 3$ matrices with octonion entries. It is endowed with the Jordan commutative multiplication:

$$
X \circ Y=\frac{1}{2}(X Y+Y X), X, Y \in \mathfrak{h}_{3}(\mathbb{C} a) .
$$

This multiplication satisfies to the identity $\left(X^{2} \circ Y\right) \circ X=X^{2} \circ(Y \circ X)$ which is the condition for an algebra with commutative (but not necessarily associative) multiplication to be Jordan. The Jordan algebra $\mathfrak{h}_{3}(\mathbb{C} a)$ is 27 -dimensional over $\mathbb{R}$. Every its element can be represented in the form:

$$
X=a_{1} E_{1}+a_{2} E_{2}+a_{3} E_{3}+X_{1}\left(\xi_{1}\right)+X_{2}\left(\xi_{2}\right)+X_{3}\left(\xi_{3}\right),
$$

where

$$
\begin{aligned}
E_{1} & =\left(\begin{array}{lll}
1 & 0 & 0 \\
0 & 0 & 0 \\
0 & 0 & 0
\end{array}\right), E_{2}=\left(\begin{array}{lll}
0 & 0 & 0 \\
0 & 1 & 0 \\
0 & 0 & 0
\end{array}\right), E_{3}=\left(\begin{array}{ccc}
0 & 0 & 0 \\
0 & 0 & 0 \\
0 & 0 & 1
\end{array}\right), \\
X_{1}(\xi) & =\left(\begin{array}{lll}
0 & 0 & 0 \\
0 & 0 & \xi \\
0 & \bar{\xi} & 0
\end{array}\right), X_{2}(\xi)=\left(\begin{array}{ccc}
0 & 0 & \bar{\xi} \\
0 & 0 & 0 \\
\xi & 0 & 0
\end{array}\right), X_{3}(\xi)=\left(\begin{array}{ccc}
0 & \xi & 0 \\
\bar{\xi} & 0 & 0 \\
0 & 0 & 0
\end{array}\right),
\end{aligned}
$$

\footnotetext{
${ }^{3}$ Other versions of this principle are in [16].
} 
$a_{i} \in \mathbb{R}, \xi_{i} \in \mathbb{C} a, i=1,2,3$. It is easy to show that

$$
\begin{aligned}
E_{i} \circ E_{j} & =\left\{\begin{array}{c}
E_{i}, \text { if } i=j, \\
0, \text { if } i \neq j,
\end{array}\right. \\
E_{i} \circ X_{j}(\xi) & =\left\{\begin{array}{c}
0, \text { if } i=j, \\
\frac{1}{2} X_{j}(\xi), \text { if } i \neq j,
\end{array}\right. \\
X_{i}(\xi) \circ X_{j}(\eta) & =\left\{\begin{array}{c}
(\xi, \eta)\left(E-E_{i}\right), \text { if } i=j, \\
\frac{1}{2} X_{i+j}(\overline{\xi \eta}), \text { if } j \equiv i+1 \bmod 3,
\end{array}\right.
\end{aligned}
$$

where $E=E_{1}+E_{2}+E_{3}$ is the unit matrix. In the last formula all indices are considered modulo 3 .

The group of all automorphisms of the Jordan algebra $\mathfrak{h}_{3}(\mathbb{C} a)$ is the exceptional simple compact 52-dimensional real Lie group $F_{4}$. This group conserves the following bilinear and trilinear functionals: $\mathcal{A}(X, Y)=\operatorname{Tr}(X \circ Y), \mathcal{B}(X, Y, Z)=\mathcal{A}(X \circ Y, Z)$. Conversely every linear operator $\mathfrak{h}_{3}(\mathbb{C} a) \mapsto \mathfrak{h}_{3}(\mathbb{C} a)$, conserving these two functionals, lies in $F_{4}$.

Define the norm of the element (51) as $\|X\|^{2}=\mathcal{A}(X, X)=\sum_{i=1}^{3}\left(a_{i}^{2}+2|\xi|^{2}\right)$. The last equality is the consequence of (52).

Theorem 2 (Freudenthal). For any $X \in \mathfrak{h}_{3}(\mathbb{C} a)$ there exists an automorphism $\Phi \in F_{4}$, such that

$$
\Phi X=\lambda_{1} E_{1}+\lambda_{2} E_{2}+\lambda_{3} E_{3},
$$

where $\lambda_{1} \geqslant \lambda_{2} \geqslant \lambda_{3}$, and the form (53) is uniquely determined by $X$. Two elements from $\mathfrak{h}_{3}(\mathbb{C} a)$ lie on the same orbit of $F_{4}$ iff their diagonal forms (53) are the same.

\subsection{The octonionic projective plane $\mathbf{P}^{2}(\mathbb{C} a)$}

Elements $X \in \mathfrak{h}_{3}(\mathbb{C} a)$ satisfying conditions

$$
X^{2}=X, \operatorname{Tr} X=1
$$

form the octonionic projective plane $\mathbf{P}^{2}(\mathbb{C} a)$, which is a 16 -dimensional real manifold. Automorphisms of $\mathfrak{h}_{3}(\mathbb{C} a)$ conserves equations (54) and the group $F_{4}$ acts on $\mathbf{P}^{2}(\mathbb{C} a)$. From the Freudenthal theorem and equations (54) it follows that every element of $\mathbf{P}^{2}(\mathbb{C} a)$ can be transformed by an appropriate element of $F_{4}$ to the element $E_{1}$. Thus $\mathbf{P}^{2}(\mathbb{C} a)$ is a homogeneous space of the group $F_{4}$ and calculations in 17] (lecture 16) shows, that the stationary subgroup of every point $X \in \mathbf{P}^{2}(\mathbb{C} a)$ is isomorphic to the group $\operatorname{Spin}(9)$.

Let

$$
X=\left(1+a_{1}\right) E_{1}+a_{2} E_{2}+a_{3} E_{3}+X_{1}\left(\xi_{1}\right)+X_{2}\left(\xi_{2}\right)+X_{3}\left(\xi_{3}\right) \in \mathbf{P}^{2}(\mathbb{C} a),
$$

where $a_{i},\left|\xi_{i}\right|, i=1,2,3$ are tending to zero. Then due to (52) we have

$$
X \circ X=\left(1+2 a_{1}\right) E_{1}+X_{2}\left(\xi_{2}\right)+X_{3}\left(\xi_{3}\right)+o\left(\sum_{i=1}^{3}\left(a_{i}^{2}+|\xi|^{2}\right)\right)
$$

and the equality $X \circ X=X$ implies $a_{1}=a_{2}=a_{3}=0, \xi_{1}=0$. It means that we can identify the tangent space $T_{E_{1}} \mathbf{P}^{2}(\mathbb{C} a)$ with the set $\left\{X_{2}\left(\xi_{2}\right)+X_{3}\left(\xi_{3}\right) \mid \xi_{1}, \xi_{2} \in \mathbb{C} a\right\}$.

Let $K \subset F_{4}$ be the stationary subgroup corresponding to the point $E_{1}$ and acting by automorphisms in the space $T_{E_{1}} \mathbf{P}^{2}(\mathbb{C} a) \simeq\left\{X_{2}\left(\xi_{2}\right)+X_{3}\left(\xi_{3}\right) \mid \xi_{1}, \xi_{2} \in \mathbb{C} a\right\}$. Let $K_{0}$ be the stationary subgroup of $K$, corresponding to the element $X_{3}(1) \in T_{E_{1}} \mathbf{P}^{2}(\mathbb{C} a)$.

According to the section [4 we are to calculate the $K_{0}$-action on $T_{E_{1}} \mathbf{P}^{2}(\mathbb{C} a)$. For any element $X \in \mathfrak{h}_{3}(\mathbb{C} a)$ let Ann $X:=\left\{Y \in \mathfrak{h}_{3}(\mathbb{C} a) \mid Y \circ X=0\right\}$. Being an automorphism of 
the algebra $\mathfrak{h}_{3}(\mathbb{C} a)$, an element $\Phi \in K_{0}$ conserves the space Ann $X_{3}(1)$. It follows from (52) that

$$
\text { Ann } X_{3}(1)=\left\{a\left(E_{1}-E_{2}\right)+b E_{3}+X_{3}(\xi) \mid a, b \in \mathbb{R}, \xi \in \mathbb{C} a^{\prime}\right\} .
$$

Let $\Phi\left(E_{1}-E_{2}\right)=a\left(E_{1}-E_{2}\right)+b E_{3}+X_{3}(\xi)$, then we have

$$
1=\mathcal{A}\left(E_{1}-E_{2}, E_{1}\right)=\mathcal{A}\left(\Phi\left(E_{1}-E_{2}\right), \Phi\left(E_{1}\right)\right)=\mathcal{A}\left(a\left(E_{1}-E_{2}\right)+b E_{3}+X_{3}(\xi), E_{1}\right)=a .
$$

This implies $\Phi\left(E_{1}-E_{2}\right)=E_{1}-E_{2}+b E_{3}+X_{3}(\xi)$ and the equality $\left\|E_{1}-E_{2}\right\|=\left\|\Phi\left(E_{1}-E_{2}\right)\right\|$ gives $b=0, \xi=0$. It means that $\Phi\left(E_{2}\right)=E_{2}$ and therefore $\Phi\left(E_{3}\right)=\Phi\left(E-E_{1}-E_{2}\right)=$ $E-E_{1}-E_{2}=E_{3}$. Thus the group $K_{0}$ conserves elements $E_{1}, E_{2}, E_{3}$.

Let $K^{\prime}$ be the subgroup of $F_{4}$ conserving element $E_{1}, E_{2}, E_{3}$. We see that $K_{0} \subset K^{\prime} \subset$ $K$. Since Ann $E_{1}=\left\{a_{2} E_{2}+a_{3} E_{3}+X_{1}(\xi), a_{1}, a_{2} \in \mathbb{R}, \xi \in \mathbb{C} a\right\}$, then the group $K^{\prime}$ maps $X_{1}(\xi) \mapsto X_{1}(\tilde{\xi})$ and similarly $X_{i}\left(\xi_{i}\right) \mapsto X_{i}\left(\tilde{\xi}_{i}\right), i=1,2,3$.

Let $\Phi_{i}: \mathbb{C} a \mapsto \mathbb{C} a, i=1,2,3$ be orthogonal operators such that $\Phi X_{i}\left(\xi_{i}\right)=X_{i}\left(\Phi_{i}\left(\xi_{i}\right)\right)$ for $\Phi \in K^{\prime}$. The last formula in (52) implies

$$
\begin{aligned}
X_{3}\left(\Phi_{3}(\overline{\xi \eta})\right) & =\Phi\left(X_{3}(\overline{\xi \eta})\right)=2 \Phi\left(X_{1}(\xi) \circ X_{2}(\eta)\right)=2 \Phi\left(X_{1}(\xi)\right) \circ \Phi\left(X_{2}(\eta)\right) \\
& =2 X_{1}\left(\Phi_{1}(\xi)\right) \circ X_{2}\left(\Phi_{2}(\eta)\right)=X_{3}\left(\overline{\Phi_{1}(\xi) \Phi_{2}(\eta)}\right) .
\end{aligned}
$$

It gives

for $\Phi \in K^{\prime}, \xi, \eta \in \mathbb{C} a$.

$$
\Phi_{1}(\xi) \Phi_{2}(\eta)=\overline{\Phi_{3}(\overline{\xi \eta})},
$$

Denote by $\mathbb{C} a_{i}, i=1,2,3$ the domains for the operators $\Phi_{i}, i=1,2,3$. Then $T_{E_{1}} \mathbf{P}^{2}(\mathbb{C} a)$ $\simeq \mathbb{C} a_{2} \oplus \mathbb{C} a_{3}$.

The formula (55) and the proposition 2 imply

Proposition 3. Operators $\Phi_{1}$ and $\Phi_{2}$ are respectively left and right spinor representations of the group $\operatorname{Spin}(8) \simeq K^{\prime}$ and the composition $\iota \circ \Phi_{3} \circ \iota$ is the vector representation of $\operatorname{Spin}(8)$.

The group Spin(8) is the universal (double) covering of the group $\mathrm{SO}(8)$ and their Lie algebras $\mathfrak{s p i n}(8)$ and $\mathfrak{s o}(8)$ are isomorphic.

Now consider representations of the Lie algebra $\mathfrak{k}^{\prime}$ of the group $K^{\prime}$ in $\mathbb{C} a_{i}, i=1,2,3$. All these representations are faithful. For $A \in \mathfrak{k}^{\prime}$ denote by $A^{(i)}$ the corresponding skew symmetric operator in $\mathbb{C} a_{i}, i=1,2,3$. From (55) we obtain the following infinitesimal analog of the triality principle:

$$
A^{(1)}(\xi) \cdot \eta+\xi \cdot A^{(2)}(\eta)=\overline{A^{(3)}(\overline{\xi \eta})} .
$$

From (49) and (50) we obtain that if $A^{(1)}=L_{i}$ (respectively $A^{(1)}=L_{i, j}$ ) then $A^{(2)}=$ $R_{i}, A^{(3)}=\iota \circ V_{i} \circ \iota$ (respectively $\left.A^{(2)}=R_{i, j}, A^{(3)}=\iota \circ V_{i, j} \circ \iota\right)$.

Let us identify the algebra $\mathfrak{k}^{\prime}$ with its vector representation in $\mathbb{C} a_{3}$, in particular we put $A \equiv A^{(3)}$ for $A \in \mathfrak{k}^{\prime}$. By $\varkappa$ denote the inclusion $\mathfrak{k}^{\prime}$ into the Lie algebra $\mathfrak{f}_{4}$ corresponding to the group $F_{4}$.

By the definition, the Lie algebra $\mathfrak{k}_{0}$ of the group $K_{0} \subset K^{\prime}$ consists of the skew symmetric operators in $\mathbb{C} a_{3}$, transforming $1 \in \mathbb{C} a_{3}$ into 0 . The group $K_{0}$ is isomorphic to Spin(7), acting in $\mathbb{C} a_{1}$ by the left spinor representation, in $\mathbb{C} a_{2}$ by the right spinor representation (equivalent for $\operatorname{Spin}(7)$ to the left one, see (63) below), and in $\mathbb{C} a_{3}^{\prime}$ by the vector representation, which are restrictions of analogous representations of $K^{\prime} \simeq \operatorname{Spin}(8)$.

Let $\mathfrak{m}$ be the space of $3 \times 3$ skew-Hermitian matrices with octonion entries and the zero trace. Let

$$
Y_{1}(\xi)=\left(\begin{array}{ccc}
0 & 0 & 0 \\
0 & 0 & \xi \\
0 & -\bar{\xi} & 0
\end{array}\right), Y_{2}(\xi)=\left(\begin{array}{ccc}
0 & 0 & -\bar{\xi} \\
0 & 0 & 0 \\
\xi & 0 & 0
\end{array}\right), Y_{3}(\xi)=\left(\begin{array}{ccc}
0 & \xi & 0 \\
-\bar{\xi} & 0 & 0 \\
0 & 0 & 0
\end{array}\right), \xi \in \mathbb{C} a
$$


be elements from $\mathfrak{m}$ and the linear subspace $\mathfrak{m}_{0} \subset \mathfrak{m}$ consists of elements of the form

$$
\sum_{i=1}^{3} Y_{i}\left(\xi_{i}\right), \xi_{i} \in \mathbb{C} a .
$$

From [17] (lecture 16) we can extract the following proposition:

Proposition 4. For $Y \in \mathfrak{m}$ the linear operator $\operatorname{ad} Y: \mathfrak{h}_{3}(\mathbb{C} a) \mapsto \mathfrak{h}_{3}(\mathbb{C} a)$, acting according to the formula ad $Y(X)=Y X-X Y, X \in \mathfrak{h}_{3}(\mathbb{C} a)$ is the differentiation of the algebra $\mathfrak{h}_{3}(\mathbb{C} a)$. Thus the space $\mathfrak{m}$ is contained in $\mathfrak{f}_{4}$. There is the expansion into the direct sum of linear spaces

$$
\mathfrak{f}_{4} \simeq \mathfrak{k}^{\prime} \oplus \mathfrak{m}_{0}
$$

with the following commutator relations

$$
\begin{aligned}
& {\left[\varkappa A, \operatorname{ad} Y_{i}(\xi)\right]=\operatorname{ad} Y_{i}\left(A^{(i)} \xi\right), i=1,2,3} \\
& {\left[\operatorname{ad} Y_{i}(\xi), \operatorname{ad} Y_{j}(\eta)\right]=\left\{\begin{array}{c}
\varkappa C_{i, \xi, \eta}, \text { if } j=i, \\
\operatorname{ad} Y_{i+2}(-\overline{\xi \eta}), \text { if } j=i+1,
\end{array}\right.}
\end{aligned}
$$

where $A \equiv A^{(3)} \in \mathfrak{k}^{\prime}, \xi, \eta \in \mathbb{C} a$, operators $A^{(i)}$ are from [56), the indices in the last equation are considered modulo 3 and skew-Hermitian operators $C_{i, \xi, \eta}: \mathbb{C} a_{3} \mapsto \mathbb{C} a_{3}, i=1,2,3$ are given by the following formulas:

$$
\begin{aligned}
& C_{1, \xi, \eta}: \zeta \mapsto \zeta \xi \cdot \bar{\eta}-\zeta \eta \cdot \bar{\xi}, \\
& C_{2, \xi, \eta}: \zeta \mapsto \bar{\eta} \cdot \xi \zeta-\bar{\xi} \cdot \eta \zeta, \zeta \in \mathbb{C} a \\
& C_{3, \xi, \eta}: \zeta \mapsto 4(\xi, \zeta) \eta-4(\eta, \zeta) \xi .
\end{aligned}
$$

The action of operators $\varkappa C_{i, \xi, \eta}$ on the spaces $\mathbb{C} a_{1}$ and $\mathbb{C} a_{2}$ is obtained from (59) by the cyclic permutation of indices:

$$
\begin{aligned}
& \left.\varkappa C_{1, \xi, \eta}\right|_{\mathbb{C} a_{1}}: \zeta \mapsto 4(\xi, \zeta) \eta-4(\eta, \zeta) \xi, \\
& \left.\varkappa C_{2, \xi, \eta}\right|_{\mathbb{C} a_{1}}: \zeta \mapsto \zeta \xi \cdot \bar{\eta}-\zeta \eta \cdot \bar{\xi}, \\
& \left.\varkappa C_{3, \xi, \eta}\right|_{\mathbb{C} a_{1}}: \zeta \mapsto \bar{\eta} \cdot \xi \zeta-\bar{\xi} \cdot \eta \zeta, \\
& \left.\varkappa C_{1, \xi, \eta}\right|_{\mathbb{C} a_{2}}: \zeta \mapsto \bar{\eta} \cdot \xi \zeta-\bar{\xi} \cdot \eta \zeta, \\
& \left.\varkappa C_{2, \xi, \eta}\right|_{\mathbb{C} a_{2}}: \zeta \mapsto 4(\xi, \zeta) \eta-4(\eta, \zeta) \xi, \\
& \left.\varkappa C_{3, \xi, \eta}\right|_{\mathbb{C a}_{2}}: \zeta \mapsto \zeta \xi \cdot \bar{\eta}-\zeta \eta \cdot \bar{\xi} .
\end{aligned}
$$

Note that in [17] (lecture 16) analogs of formulae (55), (57) and the last formula (52) contain errors.

\section{Generators of algebras Diff $\left(\mathbf{P}^{2}(\mathbb{C} a)_{\mathbb{S}}\right)$ and $\operatorname{Diff}\left(\mathbf{H}^{2}(\mathbb{C} a)_{\mathbb{S}}\right)$}

Now we are to specify the construction from section 4 for the space $M=\mathbf{P}^{2}(\mathbb{C} a)_{\mathbb{S}}$.

\subsection{The special base in $\mathfrak{a} \oplus \mathfrak{p}_{\lambda} \oplus \mathfrak{k}_{\lambda} \oplus \mathfrak{p}_{2 \lambda} \oplus \mathfrak{k}_{2 \lambda}$}

It is easily seen that

$$
\left[Y_{1}(\xi), E_{1}\right]=0,\left[Y_{2}(\xi), E_{1}\right]=X_{2}(\xi),\left[Y_{3}(\xi), E_{1}\right]=-X_{3}(\xi), \xi \in \mathbb{C} a,
$$


so we can identify the space $T_{E_{1}} \mathbf{P}^{2}(\mathbb{C} a)$ with the space $\left\{Y_{2}(\xi)+Y_{3}(\eta) \mid \xi, \eta \in \mathbb{C} a\right\} \subset \mathfrak{m}_{0}$. From (57) we obtain that the expansion

$$
\left\{Y_{2}(\xi)+Y_{3}(\eta) \mid \xi, \eta \in \mathbb{C} a\right\}=\left\{Y_{3}(\xi) \mid \xi \in \mathbb{R}\right\} \oplus\left\{Y_{2}(\xi) \mid \xi \in \mathbb{C} a\right\} \oplus\left\{Y_{3}(\xi) \mid \xi \in \mathbb{C} a^{\prime}\right\}
$$

is $\mathrm{Ad}_{K_{0}}$-invariant and by the comparison with sections 3 and 4 we can put:

$$
\mathfrak{a}:=\left\{Y_{3}(\xi) \mid \xi \in \mathbb{R}\right\}, \mathfrak{p}_{\lambda}:=\left\{Y_{2}(\xi) \mid \xi \in \mathbb{C} a\right\}, \mathfrak{p}_{2 \lambda}:=\left\{Y_{3}(\xi) \mid \xi \in \mathbb{C} a^{\prime}\right\} .
$$

Let $y=\left(E_{1}, \frac{1}{2} X_{3}(1)\right) \in \mathbf{P}^{2}(\mathbb{C} a)_{\mathbb{S}}$, where $\frac{1}{2} X_{3}(1) \in \mathbb{S}_{E_{1}}$. We have $T_{y} \mathbf{P}^{2}(\mathbb{C} a)_{\mathbb{S}}=$ $T_{E_{1}} \mathbf{P}^{2}(\mathbb{C} a) \oplus T_{\frac{1}{2} X_{3}(1)} \mathbb{S}_{E_{1}}$ and

$$
T_{\frac{1}{2} X_{3}(1)} \mathbb{S}_{E_{1}} \simeq\left\{X_{2}(\xi) \mid \xi \in \mathbb{C} a\right\} \oplus\left\{X_{3}(\xi) \mid \xi \in \mathbb{C} a^{\prime}\right\} .
$$

Since ad $Y_{1}(\xi)\left(X_{3}(1)\right)=-X_{2}(\bar{\xi}), \xi \in \mathbb{C} a$, the space $\left\{X_{2}(\xi) \mid \xi \in \mathbb{C} a\right\} \subset T_{\frac{1}{2} X_{3}(1)} \mathbb{S}_{E_{1}}$ is identified with the space $\left\{Y_{1}(\xi) \mid \xi \in \mathbb{C} a\right\} \subset \mathfrak{m}_{0}$. Since $\operatorname{dim}_{\mathbb{R}}\left\{Y_{1}(\xi) \mid \xi \in \mathbb{C} a\right\}=8=\operatorname{dim}_{\mathbb{R}} \mathfrak{p}_{\lambda}$ we are to denote $\mathfrak{k}_{\lambda}:=\left\{Y_{1}(\xi) \mid \xi \in \mathbb{C} a\right\}$. Thus $\mathfrak{m}_{0}=\mathfrak{a} \oplus \mathfrak{p}_{\lambda} \oplus \mathfrak{p}_{2 \lambda} \oplus \mathfrak{k}_{\lambda}$.

Denote by $A_{i j} \in \mathfrak{k}^{\prime}, i \neq j$ the generators of the rotation in the 2-dimensional plane, containing elements $e_{i}, e_{j} \in \mathbb{C} a_{3}$, such that $A_{i j} e_{j}=e_{i}, A_{i j} e_{i}=-e_{j}$. The operators $A_{i j}, 1 \leqslant i<j \leqslant 7$ are the base of the algebra $\mathfrak{k}_{0}$. Similar to the quaternion case the subspace $\mathfrak{q}$ of the algebra $\mathfrak{k}^{\prime}$ with the base $A_{0 \alpha}=: A_{\alpha}, \alpha=1, \ldots, 7$ is $\operatorname{Ad}_{K_{0}}$-invariant and is identified through the $K_{0}$-action on $T_{y} \mathbf{P}^{2}(\mathbb{C} a)_{\mathbb{S}}$ with the space $\left\{X_{3}(\xi) \mid \xi \in \mathbb{C} a^{\prime}\right\} \subset$ $T_{\frac{1}{2} X_{3}(1)} \mathbb{S}_{E_{1}}$. Therefore we define $\mathfrak{k}_{2 \lambda}:=\mathfrak{q}$.

Lemma 1. It holds

$$
\begin{gathered}
A_{\alpha}^{(1)}=L_{\alpha}, A_{\alpha}^{(2)}=R_{\alpha}, A_{\alpha \beta}^{(1)}=L_{\beta, \alpha}, A_{\alpha \beta}^{(2)}=R_{\beta, \alpha}, \\
C_{3, e_{\alpha}, e_{\beta}}=4 A_{\beta, \alpha}, C_{3, e_{0}, e_{\alpha}}=-4 A_{\alpha}, \alpha, \beta=1, \ldots, 7, \alpha \neq \beta
\end{gathered}
$$

Proof. From (56) we have

$$
A^{(3)}(\xi)=\overline{A^{(1)}(\bar{\xi})+\bar{\xi} A^{(2)}(1)} .
$$

Let $A^{(1)}=L_{\alpha}$, then $A^{(2)}=R_{\alpha}$ and $A^{(3)}\left(e_{k}\right)=\frac{1}{2} \overline{\left(e_{\alpha} \bar{e}_{k}+\bar{e}_{k} e_{\alpha}\right)}=-\frac{1}{2}\left(e_{k} e_{\alpha}+e_{\alpha} e_{k}\right)$. If $1 \leqslant k \neq \alpha$, then $e_{k} e_{\alpha}=-e_{\alpha} e_{k}$ and $A^{(3)}\left(e_{k}\right)=0$. Therefore $A^{(3)}=A_{\alpha}$, since $A^{(3)}(1)=-e_{\alpha}, A^{(3)}\left(e_{\alpha}\right)=1$. This proves $A_{\alpha}^{(1)}=L_{\alpha}, A_{\alpha}^{(2)}=R_{\alpha}$.

Let now $A^{(1)}=L_{\beta, \alpha}$, then $A^{(2)}=R_{\beta, \alpha}$ and $A^{(3)}\left(e_{k}\right)=\frac{1}{2} \overline{\left(e_{\beta} \cdot e_{\alpha} \bar{e}_{k}+\bar{e}_{k} \cdot e_{\alpha} e_{\beta}\right)}=$ $\frac{1}{2}\left(e_{k} e_{\alpha} \cdot e_{\beta}+e_{\beta} e_{\alpha} \cdot e_{k}\right)$. It is easy to verify by direct computation that if $\alpha=1, \beta=2$ then $A^{(3)}\left(e_{k}\right)=0$, for $k \neq 1,2$ and $A^{(3)}\left(e_{1}\right)=-e_{2}, A^{(3)}\left(e_{2}\right)=e_{1}$. Thus $L_{\beta, \alpha}^{(3)}=A_{12}$. Therefore $L_{\beta, \alpha}^{(3)}=A_{\alpha \beta}$ for any other pair of $e_{\alpha}, e_{\beta}$, since the group $G_{2}$ of automorphisms of $\mathbb{C} a$ acts transitively on any pair of imaginary units [17] (lecture 15). This proves $A_{\alpha \beta}^{(1)}=L_{\beta, \alpha}, A_{\alpha \beta}^{(2)}=R_{\beta, \alpha}$.

The last two equalities of this lemma are obvious.

Let summarize these reasoning in the following proposition:

Proposition 5. Let

$$
\begin{aligned}
\Lambda & :=\frac{1}{2} \operatorname{ad} Y_{3}\left(e_{1}\right), e_{2 \lambda, \alpha}:=\frac{1}{2} \operatorname{ad} Y_{3}\left(e_{\alpha}\right), f_{2 \lambda, \alpha}:=\varkappa A_{\alpha}, \\
e_{\lambda, i} & :=-\frac{1}{2} \operatorname{ad} Y_{2}\left(\bar{e}_{i}\right), f_{\lambda, i}:=\frac{1}{2} \operatorname{ad} Y_{1}\left(e_{i}\right), \tilde{A}_{\alpha \beta}:=\varkappa A_{\alpha \beta},
\end{aligned}
$$


where latin indices vary from 0 to 7 and greek ones (except $\lambda$ ) vary from 1 to 7 . We have the following commutator relations:

$$
\begin{aligned}
{\left[\Lambda, e_{2 \lambda, \alpha}\right] } & =-f_{2 \lambda, \alpha},\left[\Lambda, f_{2 \lambda, \alpha}\right]=e_{2 \lambda, \alpha},\left[\Lambda, e_{\lambda, i}\right]=-\frac{1}{2} f_{\lambda, i},\left[\Lambda, f_{\lambda, i}\right]=\frac{1}{2} e_{\lambda, i},\left[\Lambda, \tilde{A}_{\alpha \beta}\right]=0, \\
{\left[e_{2 \lambda, \alpha}, e_{2 \lambda, \beta}\right] } & =\tilde{A}_{\beta \alpha},\left[e_{2 \lambda, \alpha}, f_{2 \lambda, \beta}\right]=-\delta_{\alpha \beta} \Lambda,\left[f_{2 \lambda, \alpha}, f_{2 \lambda, \beta}\right]=\tilde{A}_{\beta \alpha},\left[e_{2 \lambda, \alpha}, e_{\lambda, j}\right]=\frac{1}{2} f_{\lambda, e_{\alpha} e_{j}}, \\
{\left[e_{2 \lambda, \alpha}, f_{\lambda, j}\right] } & =\frac{1}{2} e_{\lambda, e_{\alpha} e_{j}},\left[f_{2 \lambda, \alpha}, e_{\lambda, j}\right]=-\frac{1}{2} e_{\lambda, e_{\alpha} e_{j}},\left[f_{2 \lambda, \alpha}, f_{\lambda, j}\right]=\frac{1}{2} f_{\lambda, e_{\alpha} e_{j}}, \\
{\left[e_{\lambda, i}, e_{\lambda, j}\right] } & =\frac{1}{4} \varkappa C_{2, \bar{e}_{i}, \bar{e}_{j}}=\frac{1}{2} f_{2 \lambda, e_{i} \bar{e}_{j}}+\frac{1}{2} \varkappa \tilde{C}_{2, i, j}, i \neq j, \\
{\left[f_{\lambda, i}, f_{\lambda, j}\right] } & =\frac{1}{4} \varkappa C_{1, e_{i}, e_{j}}=-\frac{1}{2} f_{2 \lambda, e_{i} \bar{e}_{j}}+\frac{1}{2} \varkappa \tilde{C}_{1, i, j}, i \neq j, \\
{\left[e_{\lambda, i}, f_{\lambda, j}\right] } & =\left\{\begin{array}{c}
-\frac{1}{2} \Lambda, i=j \\
-\frac{1}{2} e_{2 \lambda, e_{i} \bar{e}_{j}}, i \neq j
\end{array},\right.
\end{aligned}
$$

where we denote $f_{\lambda, e_{\alpha} e_{j}}:=f_{\lambda, i}$ if $e_{\alpha} e_{j}=e_{i}$ and $f_{\lambda, e_{\alpha} e_{j}}:=-f_{\lambda, i}$ if $e_{\alpha} e_{j}=-e_{i}$. Analogous notation we use for $e_{\lambda, i}, e_{2 \lambda, \gamma}, f_{2 \lambda, \gamma}$. Here operators $\tilde{C}_{l, i, j}, l=1,2, i \neq j$ are in $\mathfrak{k}_{0}$ and act as:

$$
\begin{aligned}
& \tilde{C}_{1, i, j}\left(e_{k}\right)=e_{k} e_{i} \cdot \bar{e}_{j}, e_{k} \neq 1, \pm e_{i} \bar{e}_{j}, \tilde{C}_{1, i, j}\left(e_{k}\right)=0, e_{k}=1, \pm e_{i} \bar{e}_{j} \\
& \tilde{C}_{2, i, j}\left(e_{k}\right)=e_{j} \cdot \bar{e}_{i} e_{k}, e_{k} \neq 1, \pm e_{i} \bar{e}_{j}, \tilde{C}_{2, i, j}\left(e_{k}\right)=0, e_{k}=1, \pm e_{i} \bar{e}_{j} .
\end{aligned}
$$

The chosen bases $\Lambda, e_{\lambda, i}, e_{2 \lambda, \alpha}, f_{\lambda, i}, f_{2 \lambda, \alpha}$ in spaces $\mathfrak{a}, \mathfrak{p}_{\lambda}, \mathfrak{p}_{2 \lambda}, \mathfrak{k}_{\lambda}, \mathfrak{k}_{2 \lambda}$ correspond to proposition [1.

Proof. The commutator relations are easy consequences of (57), (58), lemma 1and relations in the algebra $\mathfrak{k}^{\prime} \simeq \mathfrak{s o}(8)$. For example, let us calculate the commutator $\left[f_{2 \lambda, \alpha}, e_{\lambda, j}\right]$. Actually, from (57) and lemma 1 we obtain:

$$
\begin{aligned}
{\left[f_{2 \lambda, \alpha}, e_{\lambda, j}\right] } & =-\left[\varkappa A_{\alpha}, \frac{1}{2} \operatorname{ad} Y_{2}\left(\bar{e}_{j}\right)\right]=-\frac{1}{2} \operatorname{ad} Y_{2}\left(A_{\alpha}^{(2)} \bar{e}_{j}\right)=-\frac{1}{2} \operatorname{ad} Y_{2}\left(R_{\alpha} \bar{e}_{j}\right) \\
& =-\frac{1}{4} \operatorname{ad} Y_{2}\left(\bar{e}_{j} e_{\alpha}\right)=\frac{1}{4} \operatorname{ad} Y_{2}\left(\bar{e}_{j} \bar{e}_{\alpha}\right)=-\frac{1}{2} e_{\lambda, e_{\alpha} e_{j}} .
\end{aligned}
$$

Similar calculations are also valid for $\left[f_{2 \lambda, \alpha}, f_{\lambda, j}\right]$.

Now, let us calculate $\left[e_{\lambda, i}, e_{\lambda, j}\right], i \neq j$. From (58) we obtain:

$$
\left[e_{\lambda, i}, e_{\lambda, j}\right]=\frac{1}{4}\left[\operatorname{ad} Y_{2}\left(\bar{e}_{i}\right), \operatorname{ad} Y_{2}\left(\bar{e}_{j}\right)\right]=\frac{1}{4} \varkappa C_{2, \bar{e}_{i}, \bar{e}_{j}}, i \neq j .
$$

From (47) and (59) we obtain

$$
\frac{1}{2} C_{2, \bar{e}_{i}, \bar{e}_{j}}\left(e_{k}\right)=\frac{1}{2}\left(e_{j} \cdot \bar{e}_{i} e_{k}-e_{i} \cdot \bar{e}_{j} e_{k}\right)=-e_{i} \cdot \bar{e}_{j} e_{k} .
$$

In particular,

$$
\frac{1}{2} C_{2, \bar{e}_{i}, \bar{e}_{j}}(1)=-e_{i} \bar{e}_{j}, \frac{1}{2} C_{2, \bar{e}_{i}, \bar{e}_{j}}\left(e_{i} \bar{e}_{j}\right)=-\left(e_{i} \cdot \bar{e}_{j}\right)^{2}=1,
$$

so

$$
\frac{1}{2} \varkappa C_{2, \bar{e}_{i}, \bar{e}_{j}}=\varkappa A_{e_{i} \bar{e}_{j}}+\varkappa \tilde{C}_{2, i, j}=f_{2 \lambda, e_{i} \bar{e}_{j}}+\varkappa \tilde{C}_{2, i, j}
$$

where $\tilde{C}_{2, i, j} \in \mathfrak{k}_{0}$ and

$$
\tilde{C}_{2, i, j}\left(e_{k}\right)=e_{j} \cdot \bar{e}_{i} e_{k}, e_{k} \neq 1, \pm e_{i} \bar{e}_{j}, \tilde{C}_{2, i, j}\left(e_{i} \bar{e}_{j}\right)=\tilde{C}_{2, i, j}(1)=0 .
$$

The similar calculations are also valid for $\left[f_{\lambda, i}, f_{\lambda, j}\right]$. 


\subsection{Invariants in $S\left(\mathfrak{a} \oplus \mathfrak{p}_{\lambda} \oplus \mathfrak{k}_{\lambda} \oplus \mathfrak{p}_{2 \lambda} \oplus \mathfrak{k}_{2 \lambda}\right)$}

Invariant operators $D_{0}, \ldots, D_{6}$, corresponding to some $K_{0}$-invariants in $S\left(\mathfrak{a} \oplus \mathfrak{p}_{\lambda} \oplus \mathfrak{k}_{\lambda} \oplus\right.$ $\left.\mathfrak{p}_{2 \lambda} \oplus \mathfrak{k}_{2 \lambda}\right)$ are already constructed in section 4. Here we shall construct other independent invariants of $K_{0}$-action on $S\left(\mathfrak{a} \oplus \mathfrak{p}_{\lambda} \oplus \mathfrak{k}_{\lambda} \oplus \mathfrak{p}_{2 \lambda} \oplus \mathfrak{k}_{2 \lambda}\right)$ or equivalently from $S\left(\mathfrak{p}_{\lambda} \oplus \mathfrak{k}_{\lambda} \oplus \mathfrak{p}_{2 \lambda} \oplus \mathfrak{k}_{2 \lambda}\right)$, since $\mathfrak{a}$ is an invariant one dimensional space, and corresponding invariant differential operators.

An element $\Phi \in K^{\prime}$ is from $K_{0} \subset K^{\prime}$ iff $\Phi_{3}(1)=1$ and then $\Phi_{3}(\xi)=\xi$ for any $\xi \in \mathbb{R} \subset \mathbb{C} a_{3}$. Below in this sections $\Phi \in K_{0}$. The orthogonality of $\Phi_{i}$ means that

$$
\operatorname{Re}\left(\Phi_{i}(\xi) \overline{\Phi_{i}(\eta)}\right)=\operatorname{Re}(\xi \bar{\eta}), \xi, \eta \in \mathbb{C} a_{i} .
$$

In particular, $\Phi_{i}(\xi) \overline{\Phi_{i}(\xi)}=|\xi|^{2}$ and

$$
\overline{\Phi_{i}(\xi)^{-1}}=\Phi_{i}(\xi) /|\xi|^{2} .
$$

For $\eta=\bar{\xi}$ from (55) we obtain $\Phi_{1}(\xi) \Phi_{2}(\bar{\xi})=\overline{\Phi_{3}\left(|\xi|^{2}\right)}=|\xi|^{2}$, so from (62) $\Phi_{1}(\xi)=$ $|\xi|^{2} \Phi_{2}(\bar{\xi})^{-1}=\overline{\Phi_{2}(\bar{\xi})}$ and

$$
\Phi_{1}=\iota \circ \Phi_{2} \circ \iota .
$$

Let $Q_{1}(\xi, \eta)=\operatorname{Re}(\xi \eta), \xi \in \mathbb{C} a_{1}, \eta \in \mathbb{C} a_{2}$. From (61) and (63) we get:

$$
Q_{1}\left(\Phi_{1}(\xi), \Phi_{2}(\eta)\right)=\operatorname{Re}\left(\Phi_{1}(\xi) \Phi_{2}(\eta)\right)=\operatorname{Re}\left(\Phi_{1}(\xi) \overline{\Phi_{1}(\bar{\eta})}\right)=\operatorname{Re}(\xi \bar{\eta})=Q_{1}(\xi, \eta) .
$$

Thus, $Q_{1}(\xi, \eta)$ is invariant under the $K_{0}$-action.

From proposition 3 it follows that $\Phi_{1}=g^{L}, \Phi_{2}=g^{R}, \Phi_{3}=\iota \circ g^{V} \circ \iota=g^{V}$, where $g^{L}, g^{R}, g^{V}$ are respectively left spinor, right spinor and vector representation of the group $K_{0} \simeq \operatorname{Spin}(7)$, since $\left.\iota\right|_{\mathbb{C} a_{3}^{\prime}}=-$ id. Besides, the $K_{0}$-action on $\operatorname{Im}(\xi \eta), \xi \in \mathbb{C} a_{1}, \eta \in \mathbb{C} a_{2}$ equals $g^{V}$, so $Q_{2}(\xi, \eta, \zeta):=\operatorname{Re}(\operatorname{Im}(\xi \eta) \zeta)$ is invariant under $K_{0}$-action for $\zeta \in \mathbb{C} a_{3}^{\prime}$.

According to section 4 the $K_{0}$-action on $\mathfrak{p}_{\lambda}$ is equivalent to the $K_{0}$-action on $\mathfrak{k}_{\lambda}$ and the $K_{0}$-action on $\mathfrak{p}_{2 \lambda}$ is equivalent to the $K_{0}$-action on $\mathfrak{k}_{2 \lambda}$. These equivalence is establishes by the correspondence of bases $e_{\lambda, i} \leftrightarrow f_{\lambda, i}$ and $e_{2 \lambda, \alpha} \leftrightarrow f_{2 \lambda, \alpha}$. It is also confirmed by the formulas $\left[Y_{2}(\xi), E_{1}\right]=X_{2}(\xi)$, ad $Y_{1}(\xi)\left(X_{3}(1)\right)=-X_{2}(\bar{\xi}), \xi \in \mathbb{C} a$ and (63). Therefore the analog of $\operatorname{Im}(\xi \eta), \xi \in \mathbb{C} a_{1}, \eta \in \mathbb{C} a_{2}$ in $S\left(\mathfrak{p}_{\lambda} \oplus \mathfrak{k}_{\lambda} \oplus \mathfrak{p}_{2 \lambda} \oplus \mathfrak{k}_{2 \lambda}\right) \otimes \mathbb{C} a$ is

$$
\sum_{i \neq j} f_{\lambda, i} e_{\lambda, \bar{e}_{j}} \otimes e_{i} e_{j}
$$

Thus, after the identification $\mathfrak{p}_{2 \lambda} \simeq \mathbb{C} a_{3}^{\prime}$ the invariant $Q_{2}$ gives the invariant from $S\left(\mathfrak{p}_{\lambda} \oplus \mathfrak{k}_{\lambda} \oplus \mathfrak{p}_{2 \lambda} \oplus \mathfrak{k}_{2 \lambda}\right)$

$$
\sum_{i \neq j} f_{\lambda, i} e_{\lambda, \bar{e}_{j}} e_{2 \lambda, e_{i} e_{j}}=\sum_{i \neq j} f_{\lambda, i} e_{\lambda, j} e_{2 \lambda, e_{i} \bar{e}_{j}} .
$$

Therefore we can define the invariant differential operator:

$$
D_{7}=-\frac{1}{4} \sum_{i \neq j}\left\{\left\{f_{\lambda, i}, e_{\lambda, j}\right\}, e_{2 \lambda, e_{i} \bar{e}_{j}}\right\}=\frac{1}{4} \sum_{i \neq j}\left\{\left\{f_{\lambda, j}, e_{\lambda, i}\right\}, e_{2 \lambda, e_{i} \bar{e}_{j}}\right\} .
$$

Similarly, the identification $\mathfrak{k}_{2 \lambda} \simeq \mathbb{C} a_{3}^{\prime}$ gives the invariant differential operator:

$$
D_{8}=-\frac{1}{4} \sum_{i \neq j}\left\{\left\{f_{\lambda, i}, e_{\lambda, j}\right\}, f_{2 \lambda, e_{i} \bar{e}_{j}}\right\}=\frac{1}{4} \sum_{i \neq j}\left\{\left\{f_{\lambda, j}, e_{\lambda, i}\right\}, f_{2 \lambda, e_{i} \bar{e}_{j}}\right\} .
$$


It is clear that the equation (55) remains valid after the cyclic permutation of indices $1,2,3$ :

$$
\Phi_{3}(\zeta) \Phi_{1}(\xi)=\overline{\Phi_{2}(\overline{\zeta \xi})}, \Phi_{2}(\eta) \Phi_{3}(\zeta)=\overline{\Phi_{1}(\overline{\eta \zeta})}, \xi \in \mathbb{C} a_{1}, \eta \in \mathbb{C} a_{2}, \zeta \in \mathbb{C} a_{3}^{\prime} .
$$

Define

$$
P\left(\xi, \eta, \zeta_{1}, \zeta_{2}\right):=\operatorname{Re}\left(\zeta_{1} \xi \cdot \eta \zeta_{2}\right), \zeta_{1}, \zeta_{2} \in \mathbb{C} a_{3}^{\prime} .
$$

The function $P\left(\xi, \eta, \zeta_{1}, \zeta_{2}\right)$ is invariant w.r.t. the $K_{0}$-action, since due to (64), 63) and (61):

$$
\begin{aligned}
& P\left(\Phi_{1}(\xi), \Phi_{2}(\eta), \Phi_{3}\left(\zeta_{1}\right), \Phi_{3}\left(\zeta_{2}\right)\right)=\operatorname{Re}\left(\Phi_{3}\left(\zeta_{1}\right) \Phi_{1}(\xi) \cdot \Phi_{2}(\eta) \Phi_{3}\left(\zeta_{2}\right)\right) \\
& \quad=\operatorname{Re}\left(\overline{\Phi_{2}\left(\overline{\zeta_{1} \xi}\right)} \overline{\Phi_{1}\left(\overline{\eta \zeta_{2}}\right)}\right)=\operatorname{Re}\left(\Phi_{1}\left(\zeta_{1} \xi\right) \overline{\Phi_{1}\left(\overline{\eta \zeta_{2}}\right)}\right)=\operatorname{Re}\left(\zeta_{1} \xi \cdot \overline{\overline{\eta \zeta_{2}}}\right)=P\left(\xi, \eta, \zeta_{1}, \zeta_{2}\right)
\end{aligned}
$$

Functions $P\left(\xi, \eta, \zeta_{1}, \zeta_{2}\right)$ and $P\left(\xi, \eta, \zeta_{2}, \zeta_{1}\right)$ are not independent. Indeed, the corollary 15.12 in [18] gives:

$$
\operatorname{Re}(a b \cdot c)=\operatorname{Re}(b c \cdot a)=\operatorname{Re}(c a \cdot b)=\operatorname{Re}(a \cdot b c)=\operatorname{Re}(b \cdot c a)=\operatorname{Re}(c \cdot a b), a, b, c \in \mathbb{C} a .
$$

Therefore using the Moufang identity (46) we obtain:

$$
P(\xi, \eta, \zeta, \zeta)=\operatorname{Re}(\zeta \cdot \xi \eta \cdot \zeta)=\operatorname{Re}\left(\zeta^{2} \cdot \xi \eta\right)=-\operatorname{Re}\left(|\zeta|^{2} \xi \eta\right)=-|\zeta|^{2} \operatorname{Re}(\xi \eta)=-|\zeta|^{2} Q_{1}(\xi, \eta),
$$

which means that for $\zeta_{1}=\zeta_{2}=\zeta$ invariant $P(\xi, \eta, \zeta, \zeta)$ is expressed through invariants of the second order. Using the polarization of (65) w.r.t. $\zeta$, which means the substitution $\zeta=\zeta_{1}+\zeta_{2}$, we get:

$$
P\left(\xi, \eta, \zeta_{1}, \zeta_{2}\right)+P\left(\xi, \eta, \zeta_{2}, \zeta_{1}\right)=-2\left\langle\zeta_{1}, \zeta_{2}\right\rangle Q_{1}(\xi, \eta) .
$$

It means the dependence of two invariants $P\left(\xi, \eta, \zeta_{1}, \zeta_{2}\right), P\left(\xi, \eta, \zeta_{2}, \zeta_{1}\right)$ and invariants $Q_{1}(\xi, \eta),\left\langle\zeta_{1}, \zeta_{2}\right\rangle$ of the second order. The last two invariants correspond to operators $D_{3}$ and $D_{6}$.

For constructing the invariant differential operator $D_{9}$ we shall use the invariant function

$$
P\left(\xi, \eta, \zeta_{1}, \zeta_{2}\right)-P\left(\xi, \eta, \zeta_{2}, \zeta_{1}\right)
$$

Using $\sum_{k} e_{\lambda, \bar{e}_{k}} \otimes e_{k}$ as the analog of $\eta$ we get the corresponding expression from $S\left(\mathfrak{p}_{\lambda} \oplus\right.$ $\left.\mathfrak{k}_{\lambda} \oplus \mathfrak{p}_{2 \lambda} \oplus \mathfrak{k}_{2 \lambda}\right)$ :

$$
\begin{aligned}
& \sum_{\substack{i \neq j \\
j \neq k}}\left(f_{2 \lambda, e_{j} \bar{e}_{i}} f_{\lambda, i} e_{\lambda, \bar{e}_{k}} e_{2 \lambda, \bar{e}_{k} \bar{e}_{j}}-e_{2 \lambda, e_{j} \bar{e}_{i}} f_{\lambda, i} e_{\lambda, \bar{e}_{k}} f_{2 \lambda, \bar{e}_{k} \bar{e}_{j}}\right) \\
= & \sum_{\substack{i \neq j \\
j \neq k}}\left(e_{2 \lambda, e_{i} \bar{e}_{j}} f_{\lambda, i} e_{\lambda, k} f_{2 \lambda, e_{k} \bar{e}_{j}}-f_{2 \lambda, e_{i} \bar{e}_{j}} f_{\lambda, i} e_{\lambda, k} e_{2 \lambda, e_{k} \bar{e}_{j}}\right),
\end{aligned}
$$

since for $i \neq j$ it holds $e_{j} \bar{e}_{i}=-\overline{e_{j} \bar{e}_{i}}=-e_{i} \bar{e}_{j}$.

Define the corresponding invariant differential operator as

$$
D_{9}=\frac{1}{8} \sum_{\substack{i \neq j \\ j \neq k}}\left(\left\{\left\{e_{2 \lambda, e_{i} \bar{e}_{j}}, f_{\lambda, i}\right\},\left\{f_{2 \lambda, e_{k} \bar{e}_{j}}, e_{\lambda, k}\right\}\right\}-\left\{\left\{e_{2 \lambda, e_{i} \bar{e}_{j}}, e_{\lambda, i}\right\},\left\{f_{2 \lambda, e_{k} \bar{e}_{j}}, f_{\lambda, k}\right\}\right\}\right) .
$$

Let us show that there are exactly 9 independent $K_{0}$-invariants in $S\left(\mathfrak{p}_{\lambda} \oplus \mathfrak{k}_{\lambda} \oplus \mathfrak{p}_{2 \lambda} \oplus \mathfrak{k}_{2 \lambda}\right)$. 
Indeed, $\operatorname{dim}\left(\mathfrak{p}_{\lambda} \oplus \mathfrak{k}_{\lambda} \oplus \mathfrak{p}_{2 \lambda} \oplus \mathfrak{k}_{2 \lambda}\right)=8+8+7+7=30$ and $\operatorname{dim} K_{0}=\operatorname{dim} \operatorname{Spin}(7)=21$. Therefore the codimension of $K_{0}$-orbits in $\mathfrak{p}_{\lambda} \oplus \mathfrak{k}_{\lambda} \oplus \mathfrak{p}_{2 \lambda} \oplus \mathfrak{k}_{2 \lambda}$ is at least $30-21=9$ and there should be at least 9 independent $K_{0}$-invariants in $S\left(\mathfrak{p}_{\lambda} \oplus \mathfrak{k}_{\lambda} \oplus \mathfrak{p}_{2 \lambda} \oplus \mathfrak{k}_{2 \lambda}\right)$.

From another hand, it is obvious that the stationary subgroup, corresponding to a point in a general position, of the group $\operatorname{Spin}(7)$, acting in $\mathfrak{p}_{2 \lambda} \oplus \mathfrak{k}_{2 \lambda}$ by $g^{V} \oplus g^{V}$, is $\operatorname{Spin}(5)$. Therefore the dimension of general $\operatorname{Spin}(7)$-orbits in $\mathfrak{p}_{2 \lambda} \oplus \mathfrak{k}_{2 \lambda}$ is $\operatorname{dim} \operatorname{Spin}(7)-\operatorname{dim} \operatorname{Spin}(5)=$ 11. The group $\operatorname{Spin}(5)$ is isomorphic to $U_{\mathbb{H}}(2)$, see [18, Proposition 5.1. In the section 6 the six independent invariants of the diagonal $U_{\mathbb{H}}(2)$-action in $\mathbb{H}^{2} \oplus \mathbb{H}^{2} \simeq \mathfrak{p}_{\lambda} \oplus \mathfrak{k}_{\lambda}$ were found, so general orbits of the last action are 10-dimensional, since $\operatorname{dim}_{\mathbb{R}}\left(\mathbb{H}^{2} \oplus \mathbb{H}^{2}\right)-6=10$. Thus, general $\operatorname{Spin}(7)$-orbits in $\mathfrak{p}_{\lambda} \oplus \mathfrak{k}_{\lambda} \oplus \mathfrak{p}_{2 \lambda} \oplus \mathfrak{k}_{2 \lambda}$ are $11+10=21$-dimensional, their codimension is 9 and there are exactly 9 functionally independent invariants of $\operatorname{Spin}(7)$ action in $\mathfrak{p}_{\lambda} \oplus \mathfrak{k}_{\lambda} \oplus \mathfrak{p}_{2 \lambda} \oplus \mathfrak{k}_{2 \lambda}$.

It is not known if there are any other invariants of this action, which are polynomial in $e_{\lambda, i}, f_{\lambda, i}, e_{2 \lambda, \alpha}, f_{2 \lambda, \alpha}$ and are not polynomial in $D_{1}, \ldots, D_{9}$. Such invariants should be connected with $D_{1}, \ldots, D_{9}$ by algebraic equation of a degree more than one. In the case of $\mathbf{P}^{n}(\mathbb{H})_{\mathbb{S}}, n \geqslant 3$ there is such invariant $D_{10}$ and $D_{10}^{2}$ is polynomial in $D_{1}, \ldots, D_{9}$. The operator $D_{10}$ arises in commutative relations of $D_{1}, \ldots, D_{9}$.

In the next section it is found that all commutators of operators $D_{1}, \ldots, D_{9}$ in the octonionic case are polynomial in $D_{1}, \ldots, D_{9}$. Therefore it seems probable that there is no an analog of $D_{10}$ in the octonionic case.

It is easily verified that automorphisms $\zeta_{\alpha}, \sigma$ acts on $D_{7}, D_{8}, D_{9}$ as

$$
\begin{aligned}
\zeta_{\alpha}\left(D_{7}\right) & =\cos (\alpha) D_{7}-\sin (\alpha) D_{8}, \zeta_{\alpha}\left(D_{8}\right)=\sin (\alpha) D_{7}+\cos (\alpha) D_{8}, \zeta_{\alpha}\left(D_{9}\right)=D_{9} \\
\sigma\left(D_{7}\right) & =D_{7}, \sigma\left(D_{8}\right)=-D_{8}, \sigma\left(D_{9}\right)=D_{9} .
\end{aligned}
$$

Similarly to the previous sections, in order to get the generators of the algebra $\operatorname{Diff}\left(\mathbf{H}^{2}(\mathbb{C} a)_{\mathbb{S}}\right)$ one can use Remark $\square$ and make the formal substitution:

$$
\Lambda \rightarrow \mathbf{i} \Lambda, e_{\lambda, i} \rightarrow \mathbf{i} e_{\lambda, i}, f_{\lambda, i} \rightarrow f_{\lambda, i}, e_{2 \lambda, \alpha} \rightarrow \mathbf{i} e_{\lambda, \alpha}, f_{\lambda, \alpha} \rightarrow f_{\lambda, \alpha} .
$$

This substitution produces the following substitution for the generators $D_{0}, \ldots, D_{10}$ :

$$
\begin{aligned}
& D_{0} \rightarrow \mathbf{i} \bar{D}_{0}, D_{1} \rightarrow-\bar{D}_{1}, D_{2} \rightarrow \bar{D}_{2}, D_{3} \rightarrow \mathbf{i} \bar{D}_{3}, D_{4} \rightarrow-\bar{D}_{4}, D_{5} \rightarrow \bar{D}_{5}, \\
& D_{6} \rightarrow \mathbf{i} \bar{D}_{6}, D_{7} \rightarrow-\bar{D}_{7}, D_{8} \rightarrow \mathbf{i} \bar{D}_{8}, D_{9} \rightarrow-\bar{D}_{9} .
\end{aligned}
$$

The operators $\bar{D}_{0}, \ldots, \bar{D}_{9}$ generate the algebra $\operatorname{Diff}\left(\mathbf{H}^{2}(\mathbb{C} a)_{\mathbb{S}}\right)$.

\section{Relations in algebras Diff $\left(\mathbf{P}^{2}(\mathbb{C} a)_{\mathbb{S}}\right)$ and Diff $\left(\mathbf{H}^{2}(\mathbb{C} a)_{\mathbb{S}}\right)$}

Below there are all 45 commutative relations of operators $D_{0}, \ldots, D_{9}$. An example of a calculation of such relation is in appendix A. All methods described in section 7 for calculating commutative relations were used in this case. Besides, the numeration the base elements $e_{\lambda, i}, f_{\lambda, i}, e_{2 \lambda, \alpha}, f_{\lambda, \alpha}$ by octonionic units $e_{i}, i=0, \ldots, 7$ is very convenient.

$$
\begin{aligned}
& {\left[D_{0}, D_{1}\right]=-D_{3},\left[D_{0}, D_{2}\right]=D_{3},\left[D_{0}, D_{3}\right]=\frac{1}{2}\left(D_{1}-D_{2}\right),\left[D_{0}, D_{4}\right]=-2 D_{6},} \\
& {\left[D_{0}, D_{5}\right]=2 D_{6},\left[D_{0}, D_{6}\right]=D_{4}-D_{5},\left[D_{0}, D_{7}\right]=-D_{8},\left[D_{0}, D_{8}\right]=D_{7},\left[D_{0}, D_{9}\right]=0,} \\
& {\left[D_{1}, D_{2}\right]=-\left\{D_{0}, D_{3}\right\}-2 D_{7},\left[D_{1}, D_{3}\right]=-\frac{1}{2}\left\{D_{0}, D_{1}\right\}+D_{8}+10 D_{0},\left[D_{1}, D_{4}\right]=2 D_{7},} \\
& {\left[D_{1}, D_{5}\right]=0,\left[D_{1}, D_{6}\right]=D_{8},\left[D_{1}, D_{7}\right]=\frac{1}{2}\left\{D_{1}, D_{2}-D_{4}\right\}-D_{9}-\frac{1}{2}\left\{D_{3}, D_{6}\right\}-D_{3}^{2}-5 D_{0}^{2}}
\end{aligned}
$$




$$
\begin{aligned}
& -\frac{3}{32} D_{1}-\frac{283}{32} D_{2}+\frac{19}{2} D_{4}-\frac{1}{2} D_{5},\left[D_{1}, D_{8}\right]=-\frac{1}{2}\left\{D_{3}, D_{5}\right\}-\frac{1}{2}\left\{D_{1}, D_{6}\right\}+10 D_{6} \\
& +\frac{35}{4} D_{3},\left[D_{1}, D_{9}\right]=\frac{1}{2}\left\{D_{5}, D_{7}\right\}-\frac{1}{2}\left\{D_{6}, D_{8}\right\}-\frac{189}{32}\left\{D_{0}, D_{3}\right\}-\frac{169}{16} D_{7} \text {, } \\
& {\left[D_{2}, D_{3}\right]=\frac{1}{2}\left\{D_{0}, D_{2}\right\}+D_{8}-10 D_{0},\left[D_{2}, D_{4}\right]=-2 D_{7},\left[D_{2}, D_{5}\right]=0,\left[D_{2}, D_{6}\right]=-D_{8} \text {, }} \\
& {\left[D_{2}, D_{7}\right]=-\frac{1}{2}\left\{D_{2}, D_{1}-D_{4}\right\}+D_{9}-\frac{1}{2}\left\{D_{3}, D_{6}\right\}+D_{3}^{2}+5 D_{0}^{2}+\frac{3}{32} D_{2}+\frac{283}{32} D_{1}-\frac{19}{2} D_{4}} \\
& +\frac{1}{2} D_{5},\left[D_{2}, D_{8}\right]=\frac{1}{2}\left\{D_{2}, D_{6}\right\}-\frac{1}{2}\left\{D_{3}, D_{5}\right\}+\frac{35}{4} D_{3}-10 D_{6}, \\
& {\left[D_{2}, D_{9}\right]=-\frac{1}{2}\left\{D_{5}, D_{7}\right\}+\frac{1}{2}\left\{D_{6}, D_{8}\right\}+\frac{189}{32}\left\{D_{0}, D_{3}\right\}+\frac{169}{16} D_{7},\left[D_{3}, D_{4}\right]=0,} \\
& {\left[D_{3}, D_{5}\right]=2 D_{8},\left[D_{3}, D_{6}\right]=D_{7},\left[D_{3}, D_{7}\right]=-\frac{1}{4}\left\{D_{1}+D_{2}, D_{6}\right\}+10 D_{6} \text {, }} \\
& {\left[D_{3}, D_{8}\right]=\frac{1}{2}\left\{D_{1}, D_{2}\right\}-\frac{1}{4}\left\{D_{1}+D_{2}, D_{5}\right\}-D_{9}-D_{3}^{2}-5 D_{0}^{2}-\frac{143}{32}\left(D_{1}+D_{2}\right)-\frac{1}{2} D_{4}} \\
& +\frac{19}{2} D_{5},\left[D_{3}, D_{9}\right]=\frac{1}{2}\left\{D_{4}, D_{8}\right\}-\frac{1}{2}\left\{D_{6}, D_{7}\right\}+\frac{189}{64}\left\{D_{0}, D_{1}-D_{2}\right\}-\frac{169}{16} D_{8}, \\
& {\left[D_{4}, D_{5}\right]=-2\left\{D_{0}, D_{6}\right\},\left[D_{4}, D_{6}\right]=-\left\{D_{0}, D_{4}\right\}+\frac{35}{2} D_{0},\left[D_{4}, D_{7}\right]=\frac{1}{2}\left\{D_{1}-D_{2}, D_{4}\right\}} \\
& +\frac{35}{4}\left(D_{2}-D_{1}\right),\left[D_{4}, D_{8}\right]=\frac{1}{2}\left\{D_{1}-D_{2}, D_{6}\right\}-\left\{D_{0}, D_{7}\right\}, \\
& {\left[D_{4}, D_{9}\right]=-9\left\{D_{0}, D_{6}\right\},\left[D_{5}, D_{6}\right]=\left\{D_{0}, D_{5}\right\}-\frac{35}{2} D_{0},\left[D_{5}, D_{7}\right]=\left\{D_{3}, D_{6}\right\}+\left\{D_{0}, D_{8}\right\} \text {, }} \\
& {\left[D_{5}, D_{8}\right]=\left\{D_{3}, D_{5}\right\}-\frac{35}{2} D_{3},\left[D_{5}, D_{9}\right]=9\left\{D_{0}, D_{6}\right\},\left[D_{6}, D_{7}\right]=\frac{1}{4}\left\{D_{1}-D_{2}, D_{6}\right\}} \\
& +\frac{1}{2}\left\{D_{3}, D_{4}\right\}+\frac{1}{2}\left\{D_{0}, D_{7}\right\}-\frac{35}{4} D_{3},\left[D_{6}, D_{8}\right]=\frac{1}{4}\left\{D_{1}-D_{2}, D_{5}\right\}+\frac{1}{2}\left\{D_{3}, D_{6}\right\} \\
& -\frac{1}{2}\left\{D_{0}, D_{8}\right\}+\frac{35}{8}\left(D_{2}-D_{1}\right),\left[D_{6}, D_{9}\right]=\frac{9}{2}\left\{D_{0}, D_{4}-D_{5}\right\}, \\
& {\left[D_{7}, D_{8}\right]=-\frac{1}{4}\left\{D_{0},\left\{D_{1}, D_{2}\right\}\right\}+\frac{1}{2}\left\{D_{0}, D_{3}^{2}\right\}+\frac{1}{2}\left\{D_{0}, D_{9}\right\}+\frac{1}{4}\left\{D_{1}-D_{2}, D_{8}\right\}+\frac{1}{4}\left\{D_{0}, D_{5}\right\}} \\
& +\frac{283}{64}\left\{D_{0}, D_{1}+D_{2}\right\}-\frac{175}{2} D_{0}-\frac{1}{2}\left\{D_{3}, D_{7}\right\}+5 D_{0}^{3}+\frac{1}{4}\left\{D_{0}, D_{4}\right\}, \\
& {\left[D_{7}, D_{9}\right]=\frac{1}{4}\left\{\left\{D_{0}, D_{7}\right\}, D_{6}\right\}+\frac{1}{8}\left\{D_{2}-D_{1},\left\{D_{4}, D_{5}\right\}\right\}-\frac{1}{4}\left\{\left\{D_{0}, D_{4}\right\}, D_{8}\right\}+\frac{1}{4}\left\{D_{1}-D_{2}, D_{6}^{2}\right\}} \\
& -\frac{1}{2}\left\{D_{0}, D_{8}\right\}+\frac{25}{32}\left\{D_{3}, D_{6}\right\}+\frac{185}{64}\left\{D_{1}-D_{2}, D_{4}\right\}+\frac{17}{8}\left\{D_{1}-D_{2}, D_{5}\right\} \\
& +\frac{35 \cdot 181}{128}\left(D_{2}-D_{1}\right),\left[D_{8}, D_{9}\right]=-\frac{1}{4}\left\{\left\{D_{0}, D_{6}\right\}, D_{8}\right\}-\frac{1}{4}\left\{D_{3},\left\{D_{4}, D_{5}\right\}\right\} \\
& +\frac{1}{4}\left\{\left\{D_{0}, D_{7}\right\}, D_{5}\right\}+\frac{1}{2}\left\{D_{3}, D_{6}^{2}\right\}+\frac{169}{32}\left\{D_{3}, D_{5}\right\}+\frac{45}{64}\left\{D_{1}-D_{2}, D_{6}\right\} \\
& +\frac{37}{8}\left\{D_{3}, D_{4}\right\}+\frac{5}{8}\left\{D_{0}, D_{7}\right\}-\frac{35 \cdot 177}{64} D_{3} \text {. }
\end{aligned}
$$

Using these relations it is not difficult to verify that the operator $D^{*}=D_{0}^{2}+D_{1}+D_{2}+$ $D_{4}+D_{5}$ lies in the centre of the algebra $\operatorname{Diff}\left(\mathbf{P}^{n}(\mathbb{H})_{\mathbb{S}}\right)$ in accordance with the section 4

Using substitution (66) one can obtains from relations above the commutative relations for the algebra $\operatorname{Diff}\left(\mathbf{H}^{2}(\mathbb{C} a)_{\mathbb{S}}\right)$. 


\section{Connection of algebras $\operatorname{Diff}\left(M_{\mathbb{S}}\right)$ with the two-body problem}

In the paper [5] there was found an expression of the quantum two-body Hamiltonian with a central potential $V(\rho)$ on an arbitrary two-point homogeneous space $M$ through radial differential operators and generators of an isometry group. Using the notation of the present paper we can write these expressions in the following way:

$$
\hat{H}=L_{2}+\left\{L_{1}, D_{0}\right\}+a_{0} D_{0}^{2}+\sum_{i=1}^{6} a_{i} D_{i}+V(\rho)
$$

for $M=\mathbf{P}^{n}(\mathbb{H})$ and $M=\mathbf{P}^{2}(\mathbb{C} a)$;

$$
\hat{H}=L_{2}+\left\{L_{1}, D_{0}\right\}+a_{0} D_{0}^{2}+\sum_{i=1}^{3} a_{i} D_{i}+a_{4} D_{4}^{2}+a_{5} D_{5}^{2}+a_{6}\left\{D_{4}, D_{5}\right\}+V(\rho),
$$

for $M=\mathbf{P}^{n}(\mathbb{C})$;

$$
\hat{H}=L_{2}+\left\{L_{1}, D_{0}\right\}+a_{0} D_{0}^{2}+\sum_{i=1}^{3} a_{i} D_{i}+V(\rho),
$$

for $M=\mathbf{P}^{n}(\mathbb{R}), \mathbf{S}^{n}, n \geqslant 3$ and

$$
\hat{H}=L_{2}+\left\{L_{1}, D_{0}\right\}+a_{0} D_{0}^{2}+a_{1} D_{1}^{2}+a_{2} D_{2}^{2}+\frac{1}{2} a_{3}\left\{D_{1}, D_{2}\right\}+V(\rho),
$$

for $M=\mathbf{P}^{2}(\mathbb{R}), \mathbf{S}^{2}$.

Here $\rho$ is the distance between particles, $L_{i}, i=1,2$ is some ordinary differential operator of the $i$-th order w.r.t. $\rho, a_{0}=$ const, $a_{i}, i=1, \ldots, 6$ are some functions of $\rho$ and masses of particles. The analogous expressions for noncompact spaces can be obtained by substitutions $D_{i} \rightarrow \bar{D}_{i}$ from above.

The main difference of these expressions from Euclidean case is the presence of noncommutative operators with coefficients depending on $\rho$. This difference makes the two-body problem on $M$ quite difficult. However, every common eigenfunction of generators $D_{i}$ gives an isolated ordinary differential equation for a radial part of an eigenfunction for $\hat{H}$. Using this approach some exact spectral series for two-body problem on $\mathbf{S}^{\mathbf{n}}$ were found for several potentials in [15]. For other two-point compact homogeneous spaces similar calculations should be more difficult.

\section{A Calculation of some commutative relations}

In this appendix we shall illustrate the main ideas of calculating some commutative relations. Let start from commutative relations (24) from section [7] We shall obtain some relations requiring the minimal calculations.

Let operators $D_{0}, \ldots, D_{10}$ are defined as in section [ 6 First let us consider the commutator $\left[D_{1}, D_{4}\right]$. It is not difficult to verify the following equalities for elements $A, B, C$ of an arbitrary associative algebra:

$$
\begin{aligned}
& {[A,\{B, C\}]=\{[A, B], C\}+\{B,[A, C]\},} \\
& \{\{A, B\}, C\}-\{A,\{B, C\}\}=[B,[A, C]],
\end{aligned}
$$




$$
\{\{A, B\}, C\}=2\{B, C\} A+\{[A, B], C\}+\{[A, C], B\}+[B,[A, C]] .
$$

In particular, when $C=B$, from (67) we have $\left[A, B^{2}\right]=\{[A, B], B\}$. This implies:

$$
\left[D_{1}, D_{4}\right]=\left\{\left[D_{1}, \Upsilon_{12}\right], \Upsilon_{12}\right\}+\left\{\left[D_{1}, \Omega_{12}\right], \Omega_{12}\right\}+\left\{\left[D_{1}, \Theta_{12}\right], \Theta_{12}\right\}
$$

Using (12) and (67) again, we obtain

$$
\left[D_{1}, \Upsilon_{12}\right]=\square_{1},\left[D_{1}, \Omega_{12}\right]=\square_{2},\left[D_{1}, \Theta_{12}\right]=\square_{3} .
$$

Thus

$$
\left[D_{1}, D_{4}\right]=\left\{\square_{1}, \Upsilon_{12}\right\}+\left\{\square_{2}, \Omega_{12}\right\}+\left\{\square_{3}, \Theta_{12}\right\}=2 D_{7}
$$

Using the permutation of coordinates $z_{1}$ and $z_{2}$ (or equivalently the automorphism $\sigma \circ \zeta_{\pi}$, see section [6), we obtain from (70):

$$
\left[D_{2}, D_{4}\right]=-2 D_{7} .
$$

Suppose now we already know the expressions for commutators

$$
\begin{aligned}
& {\left[D_{0}, D_{1}\right],\left[D_{0}, D_{3}\right],\left[D_{0}, D_{7}\right],\left[D_{1}, D_{2}\right],\left[D_{1}, D_{4}\right],\left[D_{1}, D_{5}\right],\left[D_{1}, D_{6}\right],\left[D_{1}, D_{7}\right],} \\
& {\left[D_{1}, D_{8}\right],\left[D_{2}, D_{6}\right],\left[D_{3}, D_{4}\right],\left[D_{3}, D_{6}\right],\left[D_{4}, D_{5}\right],\left[D_{4}, D_{6}\right],\left[D_{4}, D_{8}\right] .}
\end{aligned}
$$

Then from the Jacobi identity and (67) we have

$$
\begin{aligned}
0 & =\left[D_{1},\left[D_{8}, D_{4}\right]\right]+\left[D_{4},\left[D_{1}, D_{8}\right]\right]+\left[D_{8},\left[D_{4}, D_{1}\right]\right]=\left[D_{1}, \frac{1}{2}\left\{D_{2}-D_{1}, D_{6}\right\}+\left\{D_{0}, D_{7}\right\}\right] \\
& +\left[D_{4}, n(n-1) D_{6}-\frac{1}{2}\left\{D_{3}, D_{5}\right\}+\frac{3}{4} D_{3}-\frac{1}{2}\left\{D_{1}, D_{6}\right\}\right]-2\left[D_{8}, D_{7}\right] \\
& =\frac{1}{2}\left\{\left[D_{1}, D_{2}\right], D_{6}\right\}-\frac{1}{2}\left\{D_{1}-D_{2},\left[D_{1}, D_{6}\right]\right\}+\left\{\left[D_{1}, D_{0}\right], D_{7}\right\}+\left\{D_{0},\left[D_{1}, D_{7}\right]\right\} \\
& +n(n-1)\left[D_{4}, D_{6}\right]-\frac{1}{2}\left\{\left[D_{4}, D_{3}\right], D_{5}\right\}-\frac{1}{2}\left\{D_{3},\left[D_{4}, D_{5}\right]\right\}+\frac{3}{4}\left[D_{4}, D_{3}\right]-\frac{1}{2}\left\{D_{1},\left[D_{4}, D_{6}\right]\right\} \\
& -\frac{1}{2}\left\{\left[D_{4}, D_{1}\right], D_{6}\right\}-2\left[D_{8}, D_{7}\right]=-\frac{1}{2}\left\{\left\{D_{3}, D_{0}\right\}, D_{6}\right\}-\left\{D_{4}, D_{6}\right\}+\frac{1}{2}\left\{D_{2}-D_{1}, D_{8}\right\} \\
& +\left\{D_{3}, D_{7}\right\}+\left\{D_{0}, n(n-1) D_{4}-\frac{1}{2}\left\{D_{3}, D_{6}\right\}-\frac{1}{2}\left\{D_{1}, D_{4}\right\}+\frac{3}{8}\left(D_{1}-D_{2}\right)+D_{9}+D_{10}\right\} \\
& -n(n-1)\left\{D_{0}, D_{4}\right\}+\frac{3}{2} n(n-1) D_{0}+\left\{D_{3},\left\{D_{6}, D_{0}\right\}\right\}+\frac{1}{2}\left\{D_{1},\left\{D_{0}, D_{4}\right\}\right\}-\frac{3}{4}\left\{D_{1}, D_{0}\right\} \\
& +\left\{D_{7}, D_{6}\right\}-2\left[D_{8}, D_{7}\right]=\frac{1}{2}\left\{D_{2}-D_{1}, D_{8}\right\}+\left\{D_{3}, D_{7}\right\}+\frac{3}{8}\left\{D_{0}, D_{1}-D_{2}\right\} \\
& +\left\{D_{0}, D_{9}+D_{10}\right\}+\frac{3}{2} n(n-1) D_{0}-\frac{3}{4}\left\{D_{0}, D_{1}\right\}-2\left[D_{8}, D_{7}\right] .
\end{aligned}
$$

In the last equality we took into account the formulas

$$
\begin{aligned}
& \left\{\left\{D_{6}, D_{0}\right\}, D_{3}\right\}-\left\{D_{6},\left\{D_{0}, D_{3}\right\}\right\}+\left\{\left\{D_{0}, D_{6}\right\}, D_{3}\right\}-\left\{D_{0},\left\{D_{6}, D_{3}\right\}\right\}=\left[D_{0},\left[D_{6}, D_{3}\right]\right] \\
& +\left[D_{6},\left[D_{0}, D_{3}\right]\right]=-\left[D_{0}, D_{7}\right]-\frac{1}{2}\left[D_{6}, D_{2}-D_{1}\right]=D_{8}+\frac{1}{2}\left(-D_{8}-D_{8}\right)=0, \\
& \left\{\left\{D_{0}, D_{4}\right\}, D_{1}\right\}-\left\{D_{0},\left\{D_{4}, D_{1}\right\}\right\}=\left[D_{4},\left[D_{0}, D_{1}\right]\right]=\left[D_{4}, D_{3}\right]=0,
\end{aligned}
$$

which are consequences of (68). 
Thus we get:

$$
\begin{aligned}
{\left[D_{7}, D_{8}\right] } & =\frac{1}{4}\left\{D_{1}-D_{2}, D_{8}\right\}-\frac{1}{2}\left\{D_{3}, D_{7}\right\}+\frac{3}{16}\left\{D_{0}, D_{1}+D_{2}\right\}-\frac{1}{2}\left\{D_{0}, D_{9}+D_{10}\right\} \\
& -\frac{3}{4} n(n-1) D_{0} .
\end{aligned}
$$

Now let us demonstrate the calculation modulo $\left(U(\mathfrak{g}) \mathfrak{k}_{0}\right)^{K_{0}}$. Let $D_{0}, \ldots, D_{3}$ are generators of $\operatorname{Diff}\left(\mathbf{S}_{\mathbb{S}}^{n}\right), n \geqslant 3, \mathfrak{g}=\mathfrak{s o}(n+1), \mathfrak{k}_{0}=\mathfrak{s o}(n-1), K_{0}=\mathrm{SO}(n-1)$. Then from (67) we obtain:

$$
\begin{aligned}
{\left[D_{1}, D_{3}\right] } & =-8 \sum_{k, l=3}^{n+1}\left(\left\{\left\{\Psi_{1 k},\left[\Psi_{1 k}, \Psi_{1 l}\right]\right\}, \Psi_{2 l}\right\}+\left\{\Psi_{1 l},\left\{\Psi_{1 k},\left[\Psi_{1 k}, \Psi_{2 l}\right]\right\}\right\}\right) \\
& =4 \sum_{k, l=3}^{n+1}\left(\left\{\left\{\Psi_{1 k}, \Psi_{k l}\right\}, \Psi_{2 l}\right\}+\delta_{k l}\left\{\Psi_{1 l},\left\{\Psi_{1 k}, \Psi_{12}\right\}\right\}\right) \\
& =4 \sum_{\substack{k, l=3 \\
k \neq l}}^{n+1}\left\{\left\{\Psi_{k l}, \Psi_{1 k}\right\}, \Psi_{2 l}\right\}+4 \sum_{k=3}^{n+1}\left\{\Psi_{1 k},\left\{\Psi_{1 k}, \Psi_{12}\right\}\right\} .
\end{aligned}
$$

From formula (69) and commutative relations (12) one obtains:

$$
\begin{aligned}
& \sum_{\substack{k, l=3 \\
k \neq l}}^{n+1}\left\{\left\{\Psi_{k l}, \Psi_{1 k}\right\}, \Psi_{2 l}\right\}=\sum_{\substack{k, l=3 \\
k \neq l}}^{n+1}\left(2\left\{\Psi_{1 k}, \Psi_{2 l}\right\} \Psi_{k l}+\left\{\left[\Psi_{k l}, \Psi_{1 k}\right], \Psi_{2 l}\right\}+\left\{\left[\Psi_{k l}, \Psi_{2 l}\right], \Psi_{1 k}\right\}\right. \\
& \left.+\left[\Psi_{1 k},\left[\Psi_{k l}, \Psi_{2 l}\right]\right]\right) \equiv \sum_{\substack{k, l=3 \\
k \neq l}}^{n+1}\left(-\frac{1}{2}\left\{\Psi_{1 l}, \Psi_{2 l}\right\}+\frac{1}{2}\left\{\Psi_{2 k}, \Psi_{1 k}\right\}+\frac{1}{2}\left[\Psi_{1 k}, \Psi_{2 k}\right]\right) \bmod \left(U(\mathfrak{g}) \mathfrak{k}_{0}\right)^{K_{0}} \\
& =-\frac{1}{4} \sum_{\substack{k, l=3 \\
k \neq l}}^{n+1} \Psi_{12}=-\frac{(n-1)(n-2)}{4} \Psi_{12}=\frac{(n-1)(n-2)}{8} D_{0} .
\end{aligned}
$$

Formula (68) gives:

$$
\begin{aligned}
& \sum_{k=3}^{n+1}\left\{\Psi_{1 k},\left\{\Psi_{1 k}, \Psi_{12}\right\}\right\}=\sum_{k=3}^{n+1}\left(\left\{\left\{\Psi_{1 k}, \Psi_{1 k}\right\}, \Psi_{12}\right\}-\left[\Psi_{1 k},\left[\Psi_{1 k}, \Psi_{12}\right]\right]\right)=2\left\{\sum_{k=3}^{n+1} \Psi_{1 k}^{2}, \Psi_{12}\right\} \\
& -\frac{1}{2} \sum_{k=3}^{n+1}\left[\Psi_{1 k}, \Psi_{2 k}\right]=-\frac{1}{4}\left\{D_{0}, D_{1}\right\}+\frac{1}{4} \sum_{k=3}^{n+1} \Psi_{12}=-\frac{1}{4}\left\{D_{0}, D_{1}\right\}-\frac{n-1}{8} D_{0} .
\end{aligned}
$$

Finally, from (71) we obtain:

$\left[D_{1}, D_{3}\right]=-\left\{D_{0}, D_{1}\right\}-\frac{n-1}{2} D_{0}+\frac{(n-1)(n-2)}{2} D_{0}=-\left\{D_{0}, D_{1}\right\}+\frac{(n-1)(n-3)}{2} D_{0}$.

Calculation of $\left[D_{1}, D_{3}\right]$ for algebras $\operatorname{Diff}\left(\mathbf{P}^{n}(\mathbb{H})_{\mathbb{S}}\right)$ and $\operatorname{Diff}\left(\mathbf{P}^{n}(\mathbb{C})_{\mathbb{S}}\right)$ are analogous, but much longer.

Let us demonstrate calculations in octonionic case by one example. Below indices $i, j$ vary from 0 to 7 . Let $D_{0}, \ldots, D_{9}$ are generators of Diff $\left(\mathbf{P}^{2}(\mathbb{C} a)_{\mathbb{S}}\right), \mathfrak{g}=\mathfrak{f}_{4}, \mathfrak{k}_{0}=$ $\mathfrak{s p i n}(7), K_{0}=\operatorname{Spin}(7)$. Then from (67) and proposition 5 we obtain:

$$
\left[D_{1}, D_{3}\right]=\frac{1}{2} \sum_{i, j}\left(\left\{\left\{\left[e_{\lambda, i}, e_{\lambda, j}\right], e_{\lambda, i}\right\}, f_{\lambda, j}\right\}+\left\{e_{\lambda, j},\left\{e_{\lambda, i},\left[e_{\lambda, i},, f_{\lambda, j}\right]\right\}\right\}\right)
$$


$=\frac{1}{8} \sum_{i \neq j}\left\{\left\{\varkappa C_{2, \bar{e}_{i}, \bar{e}_{j}}, e_{\lambda, i}\right\}, f_{\lambda, j}\right\}-\frac{1}{4} \sum_{i}\left\{e_{\lambda, i},\left\{e_{\lambda, i}, \Lambda\right\}\right\}-\frac{1}{4} \sum_{i \neq j}\left\{e_{\lambda, j},\left\{e_{\lambda, i}, e_{2 \lambda, e_{i} \bar{e}_{j}}\right\}\right\}$.

Formulas (69), (60), (47) and proposition [5imply:

$$
\begin{aligned}
& \frac{1}{8} \sum_{i \neq j}\left\{\left\{\varkappa C_{2, \bar{e}_{i}, \bar{e}_{j}}, e_{\lambda, i}\right\}, f_{\lambda, j}\right\}=\frac{1}{8} \sum_{i, j}\left(2\left\{e_{\lambda, i}, f_{\lambda, j}\right\} \varkappa C_{2, \bar{e}_{i}, \bar{e}_{j}}+\left\{\left[\varkappa C_{2, \bar{e}_{i}, \bar{e}_{j}}, e_{\lambda, i}\right], f_{\lambda, j}\right\}\right. \\
+ & \left(\left\{\left[\varkappa C_{2, \bar{e}_{i}, \bar{e}_{j}}, f_{\lambda, j}\right], e_{\lambda, i}\right\}+\left[e_{\lambda, i},\left[\varkappa C_{2, \bar{e}_{i}, \bar{e}_{j}}, f_{\lambda, j}\right]\right]\right) \\
\equiv & \sum_{i \neq j}\left(-\frac{1}{16}\left\{\operatorname{ad} Y_{2}\left(\left.\varkappa C_{2, \bar{e}_{i}, \bar{e}_{j}}\right|_{\mathbb{C}_{a_{2}}} \bar{e}_{i}\right), f_{\lambda, j}\right\}+\frac{1}{16}\left\{\operatorname{ad} Y_{1}\left(\left.\varkappa C_{2, \bar{e}_{i}, \bar{e}_{j}}\right|_{\mathbb{C}_{a_{1}}} e_{j}\right), e_{\lambda, i}\right\}\right. \\
+ & \left.\frac{1}{16}\left[e_{\lambda, i}, \operatorname{ad} Y_{1}\left(\left.\varkappa C_{2, \bar{e}_{i}, \bar{e}_{j}}\right|_{\mathbb{C} a_{1}} e_{j}\right)\right]+\frac{1}{2}\left\{e_{\lambda, i}, f_{\lambda, j}\right\} f_{2 \lambda, e_{i} \bar{e}_{j}}\right) \bmod \left(U(\mathfrak{g}) \mathfrak{k}_{0}\right)^{K_{0}} \\
= & \sum_{i \neq j}\left(-\frac{1}{4}\left\{\operatorname{ad} Y_{2}\left(\bar{e}_{j}\right), f_{\lambda, j}\right\}-\frac{1}{8}\left\{\operatorname{ad} Y_{1}\left(e_{i}\right), e_{\lambda, i}\right\}-\frac{1}{8}\left[e_{\lambda, i}, \operatorname{ad} Y_{1}\left(e_{i}\right)\right]\right. \\
+ & \left.\frac{1}{4}\left(\left\{f_{2 \lambda, e_{i} \bar{e}_{j}},\left\{e_{\lambda, i}, f_{\lambda, j}\right\}\right\}-\left[f_{2 \lambda, e_{i} \bar{e}_{j}},\left\{e_{\lambda, i}, f_{\lambda, j}\right\}\right]\right)\right) \\
= & \sum_{i \neq j}\left(\frac{1}{2}\left\{e_{\lambda, j}, f_{\lambda, j}\right\}-\frac{1}{4}\left\{f_{\lambda, i}, e_{\lambda, i}\right\}-\frac{1}{4}\left[e_{\lambda, i}, f_{\lambda, i}\right]\right)+D_{8} \\
- & \frac{1}{4} \sum_{i \neq j}\left(\left\{\left[f_{2 \lambda, e_{i} \bar{e}_{j}}, e_{\lambda, i}\right], f_{\lambda, j}\right\}+\left\{e_{\lambda, i},\left[f_{2 \lambda, e_{i} \bar{e}_{j}}, f_{\lambda, j}\right]\right\}\right) \\
= & D_{8}+\sum_{i \neq j}\left(\frac{1}{4}\left\{e_{\lambda, j}, f_{\lambda, j}\right\}+\frac{1}{8} \Lambda+\frac{1}{8}\left\{e_{\lambda, e_{i} \bar{e}_{j} \cdot e_{i}}, f_{\lambda, j}\right\}-\frac{1}{8}\left\{e_{\lambda, i}, f_{\lambda, e_{i} \bar{e}_{j} \cdot e_{j}}\right\}\right) \\
= & D_{8}+\sum_{i \neq j}\left(\frac{1}{4}\left\{e_{\lambda, j}, f_{\lambda, j}\right\}-\frac{1}{8}\left\{e_{\lambda, e_{j} \bar{e}_{i} e_{i}}, f_{\lambda, j}\right\}-\frac{1}{8}\left\{e_{\lambda, i}, f_{\lambda, i}\right\}\right)+7 \Lambda=D_{8}+7 D_{0} .
\end{aligned}
$$

Similarly, from (68) and proposition 5 we get:

$$
\begin{aligned}
& -\frac{1}{4} \sum_{i}\left\{\left\{\Lambda, e_{\lambda, i}\right\}, e_{\lambda, i}\right\}=-\frac{1}{4} \sum_{i}\left(\left\{\Lambda,\left\{e_{\lambda, i}, e_{\lambda, i}\right\}\right\}+\left[e_{\lambda, i},\left[\Lambda, e_{\lambda, i}\right]\right]\right) \\
& =-\frac{1}{2}\left\{\Lambda, \sum_{i} e_{\lambda, i}^{2}\right\}+\frac{1}{8} \sum_{i}\left[e_{\lambda, i}, f_{\lambda, i}\right]=-\frac{1}{2}\left\{D_{0}, D_{1}\right\}-\frac{1}{2} D_{0} .
\end{aligned}
$$

Also

$$
\begin{aligned}
& -\frac{1}{4} \sum_{i \neq j}\left\{\left\{e_{2 \lambda, e_{i} \bar{e}_{j}}, e_{\lambda, i}\right\}, e_{\lambda, j}\right\}=-\frac{1}{4} \sum_{i \neq j}\left(\left\{e_{2 \lambda, e_{i} \bar{e}_{j}},\left\{e_{\lambda, i}, e_{\lambda, j}\right\}\right\}+\left[e_{\lambda, i},\left[e_{2 \lambda, e_{i} \bar{e}_{j}}, e_{\lambda, j}\right]\right]\right) \\
& =-\frac{1}{8} \sum_{i \neq j}\left[e_{\lambda, i}, f_{\lambda, e_{i} \bar{e}_{j} \cdot e_{j}}\right]=-\frac{1}{8} \sum_{i \neq j}\left[e_{\lambda, i}, f_{\lambda, i}\right]=\frac{7 \cdot 8}{2 \cdot 8} \Lambda=\frac{7}{2} D_{0},
\end{aligned}
$$

since $e_{2 \lambda, e_{i} \bar{e}_{j}}$ is antisymmetric and $\left\{e_{\lambda, i}, e_{\lambda, j}\right\}$ is symmetric w.r.t. $i, j$.

Thus

$$
\left[D_{1}, D_{3}\right]=D_{8}+\left(7-\frac{1}{2}+\frac{7}{2}\right) D_{0}-\frac{1}{2}\left\{D_{0}, D_{1}\right\}=D_{8}-\frac{1}{2}\left\{D_{0}, D_{1}\right\}+10 D_{0} .
$$

\section{B}

In this appendix we will prove the following theorem: 
Theorem 3. Let $M$ be a two point $G$-homogeneous Riemannian space, where $G$ is the identity component of the isometry group for $M$. For every smooth vector field $v$ on $M$ define a function $f_{v}$ on $M_{\mathbb{S}}$ by the following formula:

$$
f_{v}(y)=\hat{g}(v(x), \xi) \equiv\langle v(x), \xi\rangle,
$$

where $x \in M, \hat{g}(\cdot, \cdot) \equiv\langle\cdot, \cdot\rangle$ is the Riemannian metric on $M, \xi \in T_{x} M,\langle\xi, \xi\rangle=1, y=$ $(x, \xi) \in M_{\mathbb{S}}$. Let $D_{0} \in \operatorname{Diff}\left(M_{\mathbb{S}}\right)$ be the differential operator constructed in section 4 (for the noncompact case, see Remark [1). For every element $X \in \mathfrak{g}$ we denote by $\tilde{X}$ the corresponding Killing vector field on $M$. Then the condition $D_{0} f_{v} \equiv 0$ on $M_{\mathbb{S}}$ is equivalent to the equality $v=\tilde{X}$ for some $X \in \mathfrak{g}$. In other words, the kernel of the operator $D_{0}$ consist of functions $f_{\tilde{X}}$, where $X$ runs over the algebra $\mathfrak{g}$.

This theorem for the case $M=\mathbf{H}^{n}(\mathbb{R})$ was formulated and proved in 4 ] by the explicit coordinate calculations. Here we will prove it in the general case in a more conceptual way.

Proof. Let $K$ be the stationary subgroup corresponding to the point $x_{0} \in M, e_{0}=$ $\frac{1}{R} \tilde{\Lambda}\left(x_{0}\right) \in T_{x_{0}} M,\left\langle e_{0}, e_{0}\right\rangle=1$, where $\Lambda$ and $R$ are from Proposition 1 . The space $M_{\mathbb{S}}$ is the $G$-orbit $G y_{0}$, where $y_{0}=\left(x_{0}, e_{0}\right) \in M_{\mathbb{S}}$.

The action of $D_{0}$ on $f_{v}$ can be written in the following way [3] (theorem 4.3):

$$
\left(D_{0} f_{v}\right)\left(g y_{0}\right)=\left.\frac{d}{d t}\right|_{t=0} f_{v}\left(g \exp (t \Lambda) y_{0}\right), g \in G .
$$

Therefore,

$$
\begin{aligned}
\left(D_{0} f_{v}\right)\left(g y_{0}\right) & =\left.\frac{d}{d t}\right|_{t=0}\left\langle v\left(g \exp (t \Lambda) x_{0}\right), g \exp (t \Lambda) e_{0}\right\rangle \\
& =\left.\frac{d}{d t}\right|_{t=0}\left\langle v\left(g \exp (t \Lambda) g^{-1} g x_{0}\right),\left.g \exp (t \Lambda) \frac{d}{d \mu}\right|_{\mu=0} \exp (\mu \Lambda) x_{0}\right\rangle \\
& =\left.\frac{d}{d t}\right|_{t=0}\left\langle v\left(\exp \left(t \operatorname{Ad}_{g} \Lambda\right) g x_{0}\right),\left.\frac{d}{d \mu}\right|_{\mu=0} \exp \left(t \operatorname{Ad}_{g} \Lambda\right) \exp \left(\mu \operatorname{Ad}_{g} \Lambda\right) g x_{0}\right\rangle \\
& =\left.\frac{d}{d t}\right|_{t=0}\left\langle v\left(\exp \left(t \operatorname{Ad}_{g} \Lambda\right) g x_{0}\right),\left.\frac{d}{d \mu}\right|_{\mu=0} \exp \left(\mu \operatorname{Ad}_{g} \Lambda\right) \exp \left(t \operatorname{Ad}_{g} \Lambda\right) g x_{0}\right\rangle .
\end{aligned}
$$

Due to the transitivity of $G$-action on $M_{\mathbb{S}}$ the point $y:=(x, e):=\left(g x_{0},\left.\widetilde{\operatorname{Ad}_{g} \Lambda}\right|_{g x_{0}}\right)$ can be considered as arbitrary. Denote $W=\operatorname{Ad}_{g} \Lambda$. Then

$$
\left(D_{0} f_{v}\right)(y)=\left.\frac{d}{d t}\right|_{t=0}\langle v(\exp (t W) x), \tilde{W}(\exp (t W) x)\rangle=£_{\tilde{W}} \hat{g}(v(x), \tilde{W}(x)),
$$

where $£_{X}$ is the Lie derivative along the vector field $X$. The vector field $\tilde{W}$ is Killing, so $£_{\tilde{W}} \hat{g}=0$ and

$$
\begin{aligned}
D_{0} f_{v} & =\hat{g}\left(£_{\tilde{W}} v, \tilde{W}\right)+\hat{g}\left(v, £_{\tilde{W}} \tilde{W}\right)=\hat{g}\left(£_{\tilde{W}} v, \tilde{W}\right)=-\hat{g}\left(£_{v} \tilde{W}, \tilde{W}\right) \\
& =\frac{1}{2}\left(£_{v} \hat{g}\right)(\tilde{W}, \tilde{W})-\frac{1}{2} £_{v}(\hat{g}(\tilde{W}, \tilde{W}))=\frac{1}{2}\left(£_{v} \hat{g}\right)(\tilde{W}, \tilde{W}),
\end{aligned}
$$

due to $\hat{g}(\tilde{W}(x), \tilde{W}(x))=\hat{g}\left(g \tilde{\Lambda}\left(x_{0}\right), g \tilde{\Lambda}\left(x_{0}\right)\right)=\hat{g}\left(\tilde{\Lambda}\left(x_{0}\right), \tilde{\Lambda}\left(x_{0}\right)\right)=R^{2}$ and $£_{X} Y=[X, Y]_{c}$ where $[X, Y]_{c}$ is the commutator of vector fields $X$ and $Y$. The element $\tilde{W} \in T_{x} M$ is arbitrary, therefore from (72) we see that the condition $D_{0} f_{v}=0$ is equivalent to the equality $£_{v} \hat{g}=0$, which means that $v$ is a Killing vector field and has the form $v=\tilde{X}$ for some $X \in \mathfrak{g}$ if and only if $D_{0} f_{v} \equiv 0$. 


\section{References}

[1] S. Helgason, The surjectivity of invariant differential operators on symmmetric spaces, Ann. of Math. V.98(1973) P. 451-480.

[2] E.B. Vinberg, Commutative homogeneous spaces and co-isotropic symplectic actions, Russian Mathematical Surveys, V. 56(2001), N.1, P. 1-60.

[3] S. Helgason, Groups and Geometric Analysis, Acad. Press, Orlando, Fla. 1984.

[4] H.M. Reimann, Invariant differential operators in hyperbolic space, Comment. Math. Helvetici, V. 57(1982), P. 412-444.

[5] A.V. Shchepetilov, Two-body problem on two-point homogeneous spaces, invariant differential operators and the mass centre concept, J. Geom. Phys., to be published, arxiv: math-ph/0203050.

[6] S. Kobayashi, K. Nomizu, Foundations of differential geometry. V.2. Interscience publishers. N. Y., 1969.

[7] J. Tits, Sur certains classes d'espace homogenes de groupes de Lie, Acad. Roy. Belg. Cl. Sci. Mem. Coll., V. 29(1955), N. 3

[8] H. C. Wang, Two-point homogeneous spaces, Ann. Math., V. 55(1952), P.177-191.

[9] H. Matsumoto, Quelques remarques sur les espaces riemanniens isotropes. C. R. Acad. Sci. Paris. 1971. V. 272. P. 316-319.

[10] J. A. Wolf, Spaces of constant curvature, Univ. California Press, Berkeley, CA, 1972.

[11] I. Chavel, Riemannian symmetric spaces of rank one, Marcel Dekker, N.Y., 1972.

[12] S. Helgason, Differential geometry, Lie groups, and symmetric spaces. Acad. Press, N.Y., 1978.

[13] O.O. Loos, Symmetric spaces. V. II: Compact spaces and classification. W.A. Benjamen, Inc. New York-Amsterdam, 1969.

[14] D.H. Collingwood, Representations of rank one Lie groups. Research Notes in Mathematics, V. 137, Pitman, Boston, 1985.

[15] I.E. Stepanova, A.V. Shchepetilov. Two-body problem on spaces of constant curvature: II. Spectral properties of the Hamiltonian, Theor. Math. Phys., V.124(2000), 1265-1272.

[16] J.C. Baez, The octonions, Bull. Amer. Math. Soc., V.39(2001), 145-205.

[17] M.M.Postnikov, Lie groups and Lie algebras (in Russian), Nauka, Moscow, 1982.

[18] J.F. Adams, Lectures on exceptional Lie groups, Chicago Univ. Press, Chicago, 1996.

[19] A.L. Onishchik, Topology of transitive transformation groups. Leipzig: Johann Ambrosius Barth, 1994. 